\begin{abstract}
Title of Document:

TIME-DEPENDENT GREEN VEHICLE ROUTING PROBLEM
\end{abstract}

Golnush Masghati Amoli, 2018

Directed by:

Professor Ali Haghani

Department of Civil and Environmental Engineering

Green Vehicle Routing problem (GVRP) is a variant of standard Vehicle Routing Problem in which the environmental externalities of routing operations are minimized as a part of the routing cost. Early studies on GVRP were focused on minimizing energy consumption and pollution of internal combustion engine commercial vehicles. With the introduction of electric commercial vehicles and the increasing trend in their adoption in green logistics and last mile delivery operations a new strand of GVRP is introduced called "Alternative Fuel Vehicle Routing Problem (AFVRP)." The objective in AFVRP is to find optimal routes with minimum energy, time or money requirements for a fleet of alternative fuel vehicles while accounting for their operation limitations such as limited driving autonomy.

The goal of this dissertation is to develop a model for a Time-Dependent GVRP (TDGVRP) with a mixed fleet of electric and internal combustion engine commercial vehicles that finds the optimal fleet design for last mile delivery operations of a company and allocates minimum cost routes to each of these vehicles in order to satisfy the customer demands in a typical operation day. The routing cost includes vehicle purchase cost, energy consumption cost, early or late service penalty cost, labor cost, emission trading cost and Electric Commercial Vehicle (ECV) battery degradation cost. An extensive model is used to estimate the energy consumption of vehicles that accounts for not only 
the travel distance, but also speed, acceleration, and cargo load as contributing factors to energy consumption of vehicles. Moreover, by considering the time dependency of travel times along the network, the effect of congestion on the vehicle energy requirements is accounted for. This is very important in the context of ECVs where the energy consumption of the vehicle determines the remaining battery and driving range of the vehicle. While previous studies on GVRP focus only on the limitations of ECVs, the GVRP model proposed in this dissertation takes into account the limitations of both ECVs and Internal Combustion Commercial Vehicles (ICCVs). These limitations are in terms of limited range and higher purchase cost for electric commercial vehicles, and carbon emission limitations imposed by government regulations (e.g., Cap and Trade project) and Low Emission Zone (LEZ) penalties for ICCVs. Emission trading or LEZs are government-mandated regulations to control pollution by providing economic incentives for reducing emission of pollutants and electrifying distribution operations. This is a unique and complex model and no study in the literature has addressed this problem sufficiently. The results of the proposed model in this study can be used to illustrate the changes in the fleet design and routing of a delivery company as a result of these regulations.

A mathematical formulation is developed for the proposed Time-Dependent GVRP and numerical experiments are designed to demonstrate its capabilities. Commercial solvers like Xpress can be used to solve the proposed model on small-size problems but for large-size and real-world problems an appropriate heuristic is needed. A heuristic method that can find good solutions in reasonable time for this problem is 
developed and tested on several cases. Also, the model is applied to a large size case study to test its performance. At last a set of sensitivity analysis is performed on the problem characteristics to evaluate the heuristic's potential outcome in different situations. The results show that the proposed heuristic is performing very well and efficient and it can be used to identify the changes in fleet size and routing of last mile delivery operations as a result of green logistics policies. 


\title{
TIME-DEPENDENT GREEN VEHICLE ROUTING PROBLEM
}

\author{
By
}

\author{
Golnush Masghati Amoli \\ Dissertation submitted to the Faculty of the Graduate School of the \\ University of Maryland, College Park, in partial fulfillment \\ of the requirements for the degree of \\ Doctor of Philosophy \\ 2018
}

Advisory Committee:

Professor Ali Haghani, Chair

Professor Cinzia Cirillo

Professor Gang-Len Chang

Professor Martin Dresner

Professor Paul Schonfel 


\section{Dedication}

To my parents, brother and husband,

for their endless love, support and encouragement. 


\section{Acknowledgements}

First and foremost, I would like to express my greatest gratitude to my advisor, Dr. Ali Haghani, for his instruction, encouragement and continuous support during my graduate studies. His broad-range knowledge and insightful critiques provided excellent guidance to this dissertation.

I am also grateful to the member of advisory committee, Dr. Cinzia Cirillo, Dr. Gang-Len Chang, Dr. Martin Dresner, and Dr. Paul Schonfeld, for all their suggestions and comments on my research. Their advice and suggestions throughout the completion of this research were of great value to me.

Finally, I would like to express my deepest appreciation to my beloved parents and siblings. They supported me during my whole life and their unconditional love and encouragement made me who I am right now. Also, I am expressing my sincere love and gratitude to my lovely husband and my best friend, Navid. He stood by my side all these years and this dissertation would never have been completed without his irreplaceable love, support and patience. 


\section{Table of Contents}

Dedication

Acknowledgement

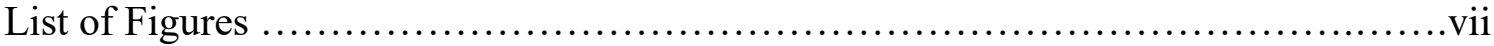

List of Tables .......................................................... viii

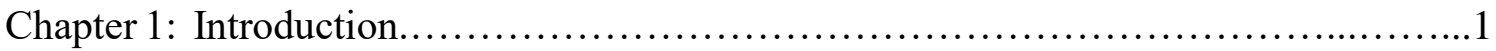

1.1Background and Motivation ..............................................

1.2 Problem Statement and Research Contributions ..................................5

1.3 Research Objectives and Scope ................................................

1.4 Research Approach ...................................................................

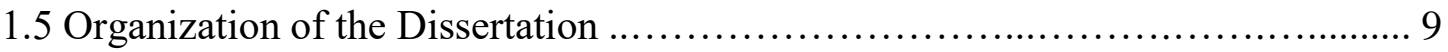



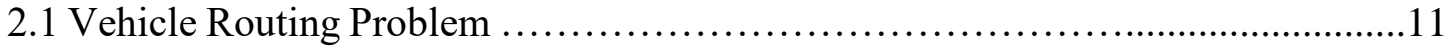

2.2 Energy Minimizing Vehicle Routing Problem ............................. 14

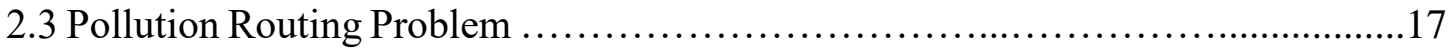

2.4 Alternative Fuel Vehicle Routing Problem .....................................20

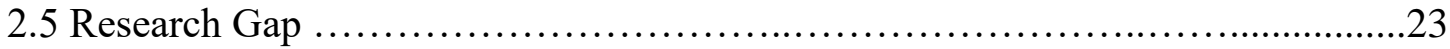

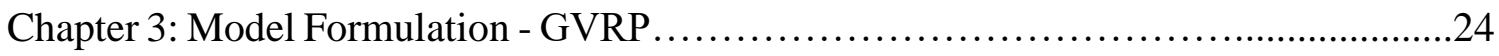

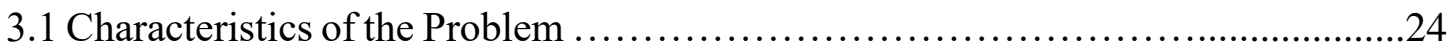



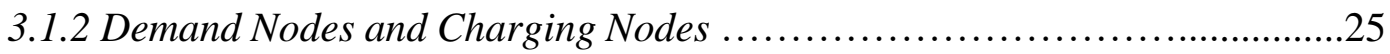

3.1.3 Commercial Vehicle Types ............................................25

3.1.4 Energy Consumption of Electric Commercial Vehicles .........................25

3.1.5 Energy Consumption of Internal Combustion Commercial Vehicles ...........29

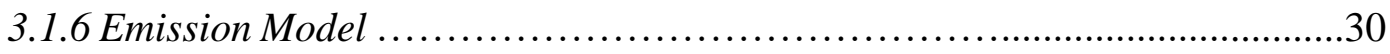

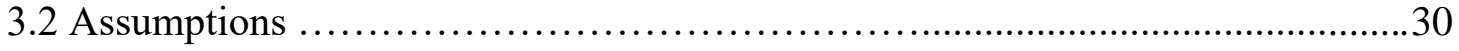

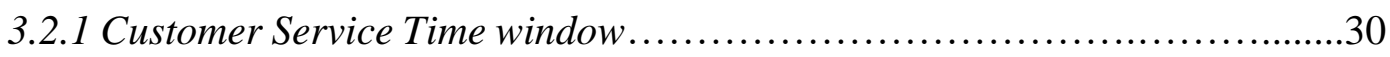

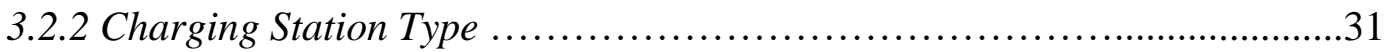

3.2.3 Vehicle Energy Consumption ......................................... 32

3.2.4 Government Incentives and Regulations ..............................32

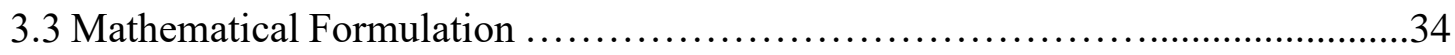

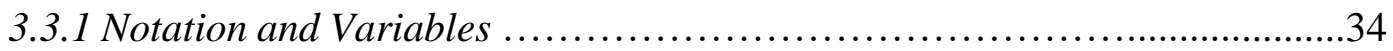

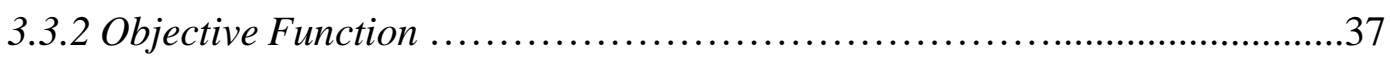

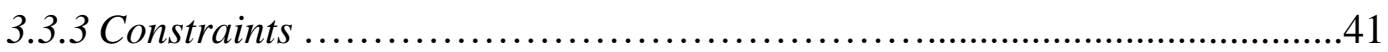

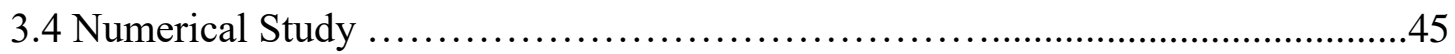






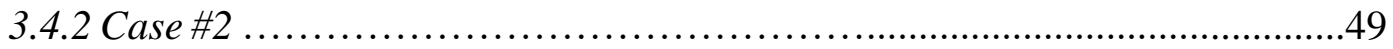

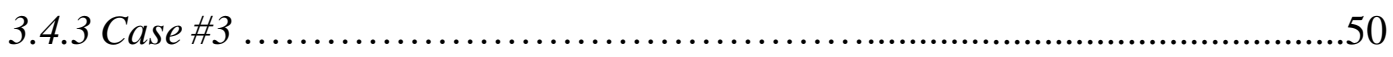



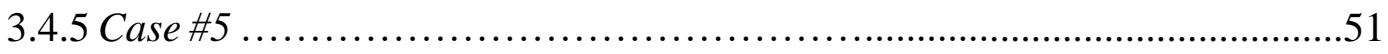

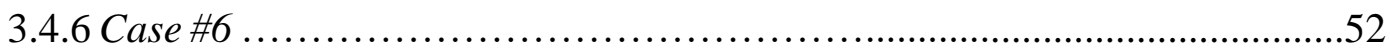

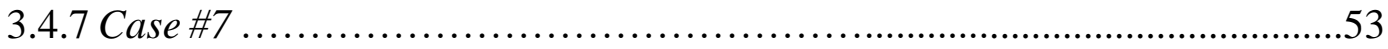

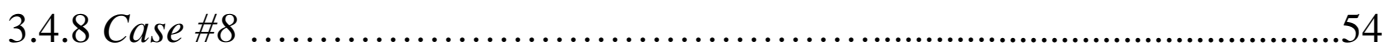





Chapter 4: Model Formulation - Time-Dependent GVRP ..........................57

4.1 Characteristics of the Problem............................................. 57

4.1.1 Network ............................................................ 57

4.1.2 Time-Dependent Travel Times .......................................58

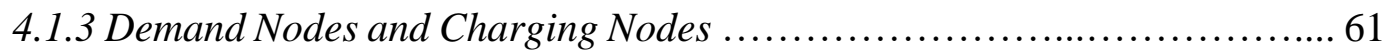

4.1.4 Commercial Vehicle Types ............................................62

4.1.5 Energy Consumption of Electric Commercial vehicles ......................62

4.1.6Energy Consumption of Internal Combustion Engine Vehicles ...............62

4.1.7 Emission Model ....................................................63

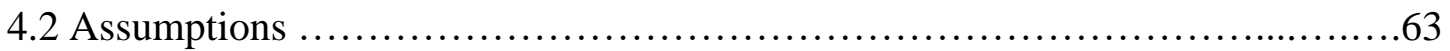

4.2.1 Customer Service Time Window ...................................63

4.2.2 Charging Station Type ..................................................63

4.2.3 Government Incentives and Regulations ............................63



4.3.1 Notation and Variables .....................................................64

4.3.2 Objective Function ......................................................67

4.3.3 Constraints ................................................................. 71



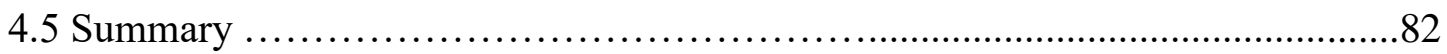



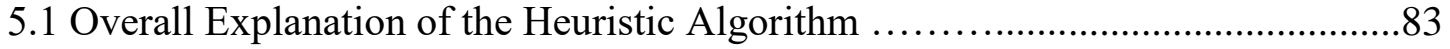

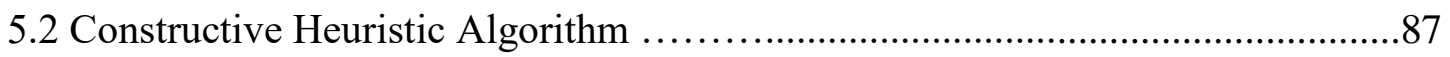

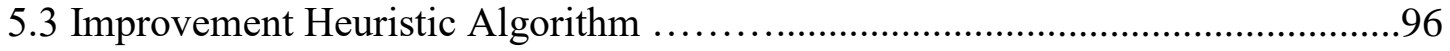

5.4 Verification of the Heuristic Algorithm for Time-Dependent GVRP ........... 102

5.5 Sensitivity Analysis on Heuristic Running Time .......................... 104

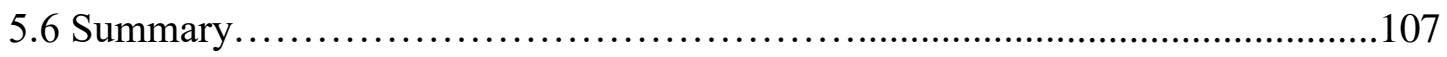

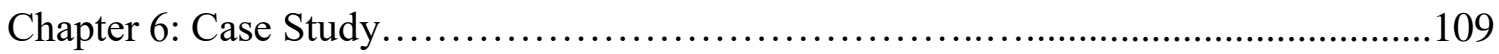

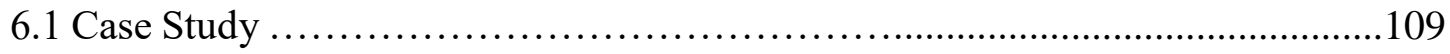


6.1.1 Problem Characteristics ...............................................109

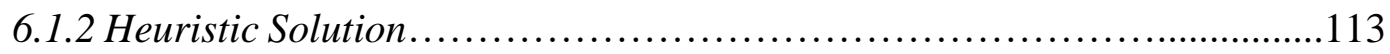

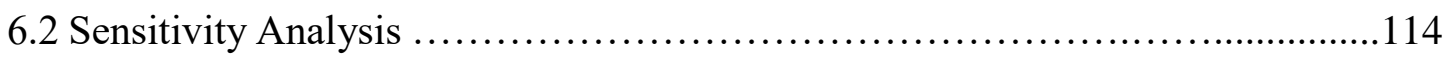



6.2.2 LEZ Coverage .....................................................117

6.2.3 LEZ Penalty Cost...................................................119

6.2.4 Emission Price and Emission Cap...................................121

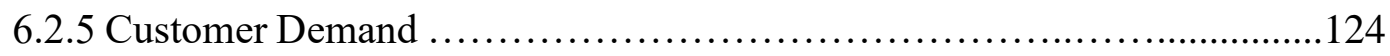

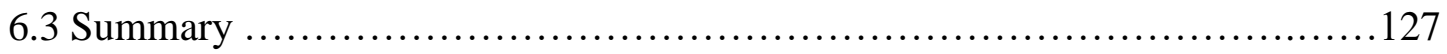

Chapter 7: Summary, Conclusions, and Recommendations for Future Research ........129

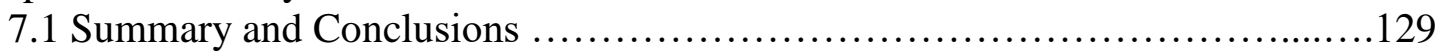

7.2 Recommendations for Future Research ................................. 133

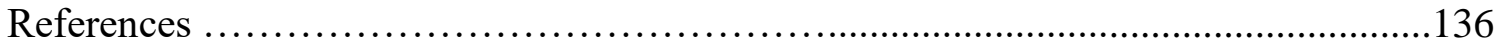




\section{List of Figures}

Figure 3. 1 The Procedure in Estimating ECV Energy Consumption .................. 26

Figure 3. 2 London LEZ Daily Charges .........................................33

Figure 3. 3 Small Size Problem Network Configurations ........................... 47

Figure 3. 4 Case\#1 Optimal Solution ........................................... 48

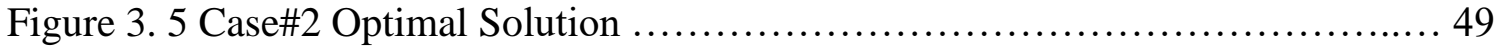

Figure 3. 6 Case\#3 Optimal Solution ....................................... 50

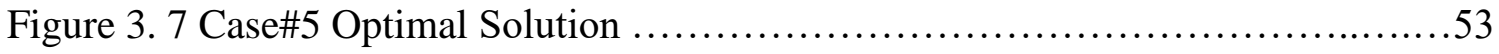

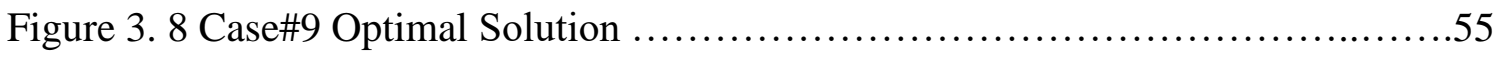

Figure 4. 1 Travel Time Step Function ..........................................59

Figure 4. 2 Travel Time as a Continuous Function of Time of a Day ...................60

Figure 4. 3 Piecewise Linear Functions of Travel Time over the Planning Period .........61

Figure 4. 4 Network of the Numerical case Study - 6 Demand Nodes.....................80

Figure 4. 5 Small Size Network _ GVRP Result .............................. 81

Figure 4. 6 Small Size Network - TDGVRP Result.............................. 82

Figure 5. 1 Constructive Heuristic Work Flow ................................. 85

Figure 5. 2 Improvement Heuristic Work Flow .................................... 86

Figure 5. 3 Sample of identical cones in a network ................................88

Figure 5. 4 Evolution of the Improvement Heuristic Algorithm - Part a .............. 100

Figure 5. 5 Evolution of the Improvement Heuristic Algorithm - Part b ...............100

Figure 5. 6 Evolution of the Improvement Heuristic Algorithm - Part c ...............101

Figure 5. 7 Evolution of the Improvement Heuristic Algorithm - Part d ...............101

Figure 5. 8 Evolution of the Improvement Heuristic Algorithm - Part e ...............102

Figure 5. 9 Sensitivity Analysis of Heuristic Running Time on Solution

Improvement .................................................. 107

Figure 6. 1 Size of the Case Study .............................................110

Figure 6. 2 Randomly Generated Network for Case Study .........................110

Figure 6. 3 Improvement in Heuristic Solution over Running Time .................. 113

Figure 6. 4 Emission Saving and Extra Cost over Different LEZ Coverage .............119

Figure 6. 5 Emission Saving and Extra Cost over Different LEZ Penalty Cost .........123

Figure 6. 6 Emission Saving and Extra Cost over Different Emission Cost - Emission

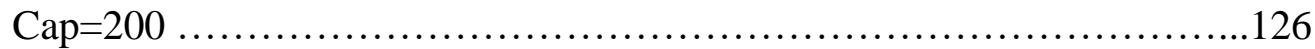

Figure 6. 7 Emission Saving and Extra Cost over Different Emission Cost - Emission

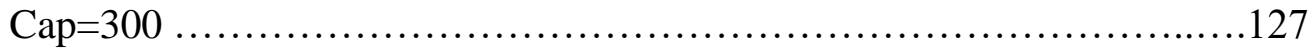




\section{List of Tables}

Table 3. 1 Case Studies Specifications ..........................................47

Table 5. 1 Characteristics of Small Size Problems ..................................... 105

Table 5. 2 Comparison Between Xpress Solver and the Heuristic Method

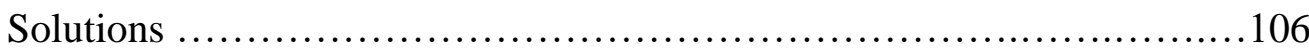

Table 6. 1 Characteristics of the Case Study .................................... 112

Table 6. 2 Characteristics of the Available Vehicles ................................113

Table 6. 3 Solution to the Case Study ...........................................114

Table 6. 4 Characteristics of the Base Scenario ..................................... 116

Table 6. 5 Solution to the Base Scenario ...........................................116

Table 6. 6 Characteristics of the Cases for LEZ Coverage Sensitivity Analysis .........118

Table 6. 7 LEZ Coverage Sensitivity Analysis Result .................................119

Table 6. 8 Characteristics of the Cases for LEZ Penalty Cost Sensitivity Analysis .....121

Table 6. 9 LEZ Penalty Cost Sensitivity Analysis Result ......................... 122

Table 6. 10 Characteristics of the Cases for Emission Price Sensitivity Analysis .......124

Table 6. 11 Emission Cost Sensitivity Analysis Result - Emission Cap $=200 \ldots \ldots \ldots .125$

Table 6. 12 Emission Cost Sensitivity Analysis Result - Emission Cap $=300 \ldots \ldots \ldots .126$

Table 6. 13 Result of Sensitivity Analysis on Demand ............................. 127 


\section{Chapter 1}

\section{INTRODUCTION}

\subsection{Backgrounds and Motivation}

Traditionally, Vehicle Routing Problems (VRPs) aim to determine the low cost routes for a fleet of vehicles to serve a set of customers such that the money and time spent to travel to those customer locations are minimized. However, the operation cost of the routing vehicles are not limited to time and money. There are environmental costs associated to these operations that had almost been neglected before the $21^{\text {st }}$ century until the substantial growth in Greenhouse Gas (GHG) emissions and the world's climate change triggered the government awareness of the urgency to conserve the environment.

The most widely cited prediction of how the world's climate might change in 21 st century was made by the Working Groups of the Intergovernmental Panel on Climate Change (IPCC) in their 2nd Assessment in 1995. This report showed that during the 20th century the average temperature of the world's climate had increased by 0.6 degrees centigrade, and it would increase by between 1.4 and 5.8 degrees centigrade by 2100 if there would be no changes in current human activities. On the other hand, the IPCC Fourth Assessment Report provided considerable evidence for the growing human influence on the climate system. It was shown that more than half of the observed increase in global average surface temperature from 1951 to 2007 was likely caused by the anthropogenic

increase in GHG concentrations. The anthropogenic influences have likely contributed to 
the retreat of glaciers since the 1960s and to the increased surface melting of the Greenland ice sheet since 1993. Transportation is one of the significant sources of anthropogenic GHG emissions. Based on GHG emission reporting guidelines, the transportation sector directly accounted for about 27 percent of total U.S. GHG emissions in 2013, increased by 16 percent since 1990 . Nearly 97 percent of transportation GHG emissions came through direct combustion of fossil fuels with freight trucks playing as the third largest source of transportation GHG emissions. The increase in the transportation related GHGs is largely due to the increased demand for travel and an increase in the number of vehicle miles traveled by passenger cars and light-duty trucks due to population growth, economic growth, urban sprawl, and low fuel prices.

Following the substantial growth in GHG emissions the government's awareness of the urgency to tackle these problems and conserve the environment increased, and green logistics received increased attention from governments and business organizations. The motivation behind green logistics was the unsustainable current production and distribution logistics strategies in the long-term. As part of the green logistics program, carriers started to use Alternative Fuel Vehicles (AFVs), for last-mile deliveries in order to reduce their GHG emissions. In North America, large companies such as FedEX, General Electric, Coca-Cola, UPS, Frito-Lay, Staples, Enterprise, and Hertz (Electrification Coalition, 2013) started introducing battery electric delivery vehicles to their last-mile delivery fleet. While this focus on truck conversion was desirable because medium- and heavy-duty trucks contribute nearly $19.2 \%$ of US transportation-based GHG emissions and the truck traffic has had the greatest growth rate of all vehicle traffic 
between 1990 and 2006 (US DOT, 2010), many companies were still reluctant to adopt ECVs in their delivery fleet due to their high purchase cost and limited autonomy. Davis and Figliozzi (2013) compared the whole life cost of battery electric delivery trucks with that of a conventional ICCV serving less-than-truckload delivery routes and the result of their analysis showed that the ECVs total cost of operation was higher $86 \%$ of the times. It was concluded that a combination of factors such as high utilization rates, low speeds and congestion, financial incentives or technological breakthrough to reduce purchase cost would make ECVs a viable alternative to ICCVs. Taefi et al. (2014) also argued that at this stage incentives are likely to be needed to increase the commercial use of the BEVs. Government incentives could be in different forms from financial incentives such as subsidizing the purchase cost of ECVs and tax exemption incentives to providing access to inner-city areas with noise or pollution limits. Imposing emission caps on industry operations could also be another lever to promote the commercial use of BEVs.

\section{- Financial Incentives}

The objective of financial incentives is to reduce the purchase cost of ECVs and the cost of providing its required charging infrastructure. One form of financial incentives is the purchase subsidies granted upon buying commercial electric vehicles. In the United States, the California Hybrid Truck and Bus Voucher Incentive Project and the New York Truck Voucher Incentive Program respectively provide up to $\$ 50,000$ and $\$ 60,000$ towards electric truck purchases not exceeding the price difference of these vehicles and the corresponding ICCVs. In Europe, Amsterdam subsidizes up to $€ 40,000$ for the purchase of electric trucks (den Boer et al. (2013), and UK covers $\% 20$ of the cost of battery electric 
vans up to $£ 8,000$ (McMorrin et al. (2012)). Financial incentives could also be in the form of exemptions from purchase value added tax (VAT), vehicle registration taxes, and fuel consumption taxes or in the form of subsidies for installation of charging equipment and infrastructure (AustriaTech (2014)).

\section{- Prioritized access incentives}

While financial incentives focus on reducing the fixed cost of electric truck adoptions, the prioritized access incentives are aimed to reduce the daily operation costs such as travel cost and travel time. These incentives could be in the form of exemption from road tolls or access to High Occupancy Lanes (HOV), bus lanes, and Low Emission Zones (LEZ). Low Emission Zones are zones in the city centers with emission limitations to promote the use of cleaner vehicles and are currently in practice in European countries such as UK, Germany, Sweden, Netherlands, Denmark and Italy. There are annual or daily fees for conventional trucks to enter these zones from which electric trucks could be exempted. Moreover, electric trucks can benefit from extended delivery time windows due to their noiseless operations. For example, in some cities only noiseless trucks have the permission to enter the city centers between $10 \mathrm{pm}$ and $7 \mathrm{am}$. Finally, free parking spaces or designated loading and unloading docks could be another incentive to encourage the use of commercial electric vehicles.

\section{- Emission Trading}

Emission trading or Cap-and-Trade is a government-mandated approach to control and reduce industry pollutants by providing financial incentives. Through this program, a cap or limit is imposed on the total amount of emissions of a specific pollutant by a 
government authority. This specified limit is then allocated among different producers of the pollutant in the form of fungible permits representing the right to release a specific quantity of the pollutant. The permits are tradable meaning that emitters who produce less than the limit are allowed to sell their extra permits to other producers. Cap-and-Trade is currently in practice in a number of countries included in the Kyoto Protocol.

Therefore, while electric commercial vehicles offer some challenges due to a limited driving range and high acquisition costs, the government regulation and incentives could justify the benefit in their adoption for distribution operations.

\section{$\underline{1.2 \text { Problem Statement and Research Contributions }}$}

The Time-Dependent Green Vehicle Routing Problem studied in this dissertation is an extension of the classic vehicle routing problem aimed at controlling the environmental externalities associated with routing a mixed fleet of ECVs and ICCVs while addressing the limitations in employment of both types of vehicles. The first study in Green Vehicle Routing Problem with a fleet of Alternative Fuel Vehicles was done by Erdogan and Miller-hooks in 2012. Since then different studies have been conducted to improve the existing models by incorporating more realistic assumptions such as vehicle load capacity limitations, customer service time windows, mixed fleet of electric and combustion engine trucks and different charging policies.

While the models developed in previous studies have been mainly focused on the limitation of the electric trucks, in this dissertation a more comprehensive GVRP is studied 
which considers the tradeoffs between the use of ECVs and ICCVs by accounting for the limitations and advantages associated to the use of both vehicle types. These limitations are in terms of limited range and higher purchase cost for ECVs, and the emission limitations imposed by the government and the Low Emission Zone penalties for ICCVs.

As another contribution and due to the important role of travel time on vehicle energy requirements, a Time-Dependent GVRP is formulated in this dissertation. Accounting for travel time variations enables the model to account for congestion level on each arc in finding optimal routes. This enhances the reliability of the model especially in routing ECVs as electric vehicle routes are very sensitive to energy consumption estimations because of their limited driving range.

Moreover, contrary to previous studies a heterogeneous fleet of ECVs and ICCVs with different battery and load capacities are considered in the problem. The route plans are not determined for a pre-specified number of vehicles. It is assumed that the number of vehicles is not predefined and the optimal number of vehicles of each type is to be found. Soft service time windows are considered in this research, which allow the vehicles to arrive at the demand nodes and start the service before or after the required service times. However, in cases that time windows are violated, the vehicles incur a penalty for late or early arrival. 


\subsection{Research Objectives and Scope}

The major goals of this research are to formulate a Fleet Size Mixed Fleet Time-Dependent Green Vehicle Routing Problem with Soft Time Windows as described in Section 1.2 and to find a proper solution algorithm for it. In order to achieve this goal, the following objectives will be pursued:

- Develop a mathematical model for the problem that will be used to find the optimal solution to the problem. The optimal solution will be the standard for testing the accuracy of the heuristic algorithm result.

- Develop an efficient heuristic algorithm capable of finding a reasonable solution to the problem within a reasonable time, so that it can be used in real life delivery routing and scheduling problems on large networks.

- Apply the proposed heuristic to solve the problem for a daily parcel delivery operation in a real size network.

- Perform sensitivity analysis on model parameters (such as percentage of demand nodes in LEZ, LEZ penalty cost, carbon permit cost, emission cap, and the customer demand at each node).

\subsection{Research Approach}

The first step of this research is to formulate the problem described in Section 1.2. This formulation step is very important, because a good formulation with fewer variables and constraints can reduce the calculation time for the exact solution. 
Next, a proper heuristic algorithm for the TDGVRP is developed. As it is well known, vehicle routing problems are NP-hard, and especially in the case of TimeDependent VRP we cannot expect the exact solution because the size of the problem increases exponentially as the number of demand nodes increases. Moreover, the soft time windows constraint, the penalty from the service time window and low emission zones violations, and carbon emission limits make this problem even more complicated. A heuristic method for the problem formulated in this research is proposed which carefully considers the calculation time as well as the accuracy of results.

The third step involves model testing by comparing the results from the developed heuristic algorithm with the results from solving the mathematical model with commercial solvers. To do so, a set of small network problems is randomly generated and the optimal solutions to these problems are found by Xpress commercial solver. The results of heuristic algorithm are compared to optimal solutions to verify the accuracy the heuristic results.

Once the efficiency of heuristic method in finding sound solutions is verified, the heuristic method is applied to solve the TDGVRP on a large size network to explore its capabilities in finding solutions to real world problems. Then a set of sensitivity analysis is performed. There are several parameters in the formulation of the TDGVRP and we test the heuristic by analyzing the sensitivity of results with respect to changes in various model parameters on a large size network. 


\subsection{Organization of the Dissertation}

The organization of the dissertation is as follows:

- Chapter 1 introduced the background and the motivation for this research. It also presented the problem statement and the research approach.

- In Chapter 2, the literature on Green Vehicle Routing Problem is reviewed. First studies on different variants of VRP are discussed. Then studies on GVRP are reviewed.

- Chapter 3 presents a proposed formulation of Fleet Size Mixed Fleet Green Vehicle Routing Problem with Soft Time Windows where travel times are assumed to be constant over the planning period.

- Chapter 4 presents the extension of the proposed formulation in Chapter 3 for the case of Time-Dependent GVRP defined in this dissertation.

- Chapter 5 presents the proposed heuristic algorithm to solve the Time-Dependent GVRP. We describe the algorithm required to generate an initial feasible solution to the problem, followed by the algorithm for solution improvement.

- Chapter 6 presents the results of the implementation of the developed heuristic method on a large size case study followed by the results of sensitivity analysis for several model parameters on a large size network.

- Finally, Chapter 7 presents summary, conclusions and recommendation for future research. 


\section{Chapter 2}

\section{LITERATURE REVIEW}

The research on the Green Vehicle Routing Problem (GVRP) deals with the optimization of the energy consumption and, as a result, pollution of the logistics transportation activities. It deals with the decisions related to the routing and scheduling of vehicles, and the choice of vehicle type for given deliveries particularly in relation to the potential added cost of $\mathrm{CO} 2$ emissions. Studies on Green Vehicle Routing Problem can be divided into two categories. The first category is the VRP in which fuel consumption and emissions of combustion engine vehicles are minimized as part of their routing costs. This type of GVRP is called Energy Minimizing Vehicle Routing Problem (EMVRP) or Pollution Routing Problem (PRP). The second category of GVRPs deals with energy consumption and also limitations of routing a fleet of alternative fuel vehicles. This type of GVRP is called Alternative Fuel Vehicle Routing Problem (AFVRP), in general, or Electric Vehicle Routing Problem (EVRP) in particular when the fleet of AFVs is of the battery electric type. In this chapter first an overview of the classic Vehicle Routing Problem and its variants is given. Then the studies on the Energy Minimizing VRP are reviewed followed by Pollution Routing Problem in the next section. Finally the literature on Alternative Fuel Vehicle Routing Problem is discussed. 


\section{$\underline{2.1 \text { Vehicle Routing Problem }}$}

The classic vehicle routing problem aims to determine the optimal routes for a fleet of homogeneous vehicles to serve a set of customers such that the time and money spent for the operation is minimized, each vehicle's route starts and ends at the depot and each customer is visited only by one vehicle. Clarke and wright proposed the first heuristic for the approximate solution of VRP in 1964 and since then numerous studies have been devoted to find the exact or approximate solutions to different variants of VRP. The most studied variant of VRP is Capacitated Vehicle Routing Problem (CVRP) in which the loading capacity of vehicles is considered as a constraint to the problem. The CVRP can be traced back to the study done by Dantzig and Ramser in 1959. Another variant is the Time-dependent Vehicle Routing Problem (TDVRP) which, in contrast to traditional VRP, assumes that the travel time between any pair of nodes in the network depends on the distance between the points and the time of day. The first formulation of the TDVRP was proposed by Malandraki and Daskin (1992) as a mixed integer linear programing model with the travel time modeled with a step function within different periods of a day. The TDVRP accounts for the fluctuations in the travel time due to urban congestion which makes it a useful model to reveal traffic congestion problems (Lecluyse, Sörensen, and Peremans (2013)) and to explore how to avoid them (Kok, Hans, and Schutten (2012)). It also helps in finding greener routes with less fuel consumption and emission by avoiding congestion. The TDVRP was further extended by considering service time windows, named as TDVRP with Time Windows (TDVRPTW). Some of the studies on TDVRPTW includes Solomon (1987); Chen, Hsueh, and Chang (2006); Soler, Albiach, and Martínez 
(2009); Kuo (2010); Maden, Eglese, and Black (2010); Figliozzi (2012); and Kritzinger et al. (2012). Pickup and Delivery Problem (PDP) is another popular strand of VRP (Wilson and Weissberg, 1967) which includes VRP with backhauls, VRP with pickup and delivery, VRP with simultaneously pickup and delivery, and dial-a-ride problem. In 1969 Tillman introduced Multi Depot VRP (MDVRP) in which more than one depot was considered in the network and the customers were visited by vehicles assigned to one of these depots. It helped the VRP to model real world delivery operations and it was adopted as an extension to other variants of VRP such as MDVRP with Time Windows (Giosa, Tansini, and Viera (2002); Polacek, et al. (2004); Dondo and Cerdá (2007)), MDVRP with Pickup and Delivery (Nagy and Salhi (2005)), or MDVRP with Mix Fleet (Salhi and Sari (1997); Salhi, Imran, and Wassan (2013)). Mixed Fleet VRP (MFVRP) is a more realistic variant of the VRP in which a heterogeneous fleet of vehicles with different purchase cost, operation cost, or loading capacity are considered for routing. Heterogeneous VRPs are rooted in the seminal paper of Golden, Assad, and Gheysens (1983) and have evolved into an extensive research area. Heterogeneous VRPs itself can be divided to different categories, namely, Fleet Size and Mixed Fleet VRP (FSMVRP), and heterogeneous Fixed Fleet VRP. The objective of FSMVRP is to minimize the fixed and variable vehicle costs by determining the optimal fleet design and assigning vehicle to minimum cost routes, while the other one aims to minimize the variable routing cost of a set of fixed given vehicles.

All the variants of the VRP mentioned above assume that the optimal location of the depots are given in the network and the developed model only looks for the optimal 
routes assigned to the vehicles. However, if the depot location is not given and it should be found through optimization, it has been observed that the design of depot location and vehicle routes separately will result into suboptimal solution with extra cost. To overcome the inefficiency in using separate models, Location Routing Problem (LRP) was introduced by Watson-Gandy and Dohm, (1973). LRP aims to jointly find the optimal location of a single or a set of depots and design a number of routes for each opened depot while the total cost of opening depots and routing is minimized. The application of LRP can be found in different operations such as waste collection, mobile communications access networks, parcel delivery, and grocery distribution (Baldacci, Mingozzi, \& Calvo (2011)).

Dynamic Vehicle Routing Problem (DVRP) is another type of VRP which deals with uncertainties in travel time, customer sets or demand, as these elements, contrary to the classic VRP assumption, are not deterministic in real world and remain inconstant during the execution of the plan. Various classes of DVRP include DVRP with Time Windows (Madsen, Tosti, and Vælds (1995); Gendreau, Guertin, Potvin, and Taillard (1999); Haghani and Jung (2005); Chen and Xu (2006); and Hong (2012)), and DVRP with Pickup and Delivery and Time Windows (Yang, Jaillet, and Mahmassani (2004); Gendreau, Guertin, Potvin, and Séguin (2006); Cheung, Choy, Li, Shi, and Tang (2008)). On the other hand, the randomness in the components of VRP is addressed by Stochastic VRP (SVRP) introduced by Gendreau, Laporte, \& Séguin in 1996. In this type of VRP some elements like customer demand, travel times, and even the set of customers in the routing problem are assumed to be random with known probabilities and the probability 
theory is the main approach to represent the uncertainty in mathematical models in this context. Some of the studies on SVRP include VRP with stochastic demand (Golden and Stewart (1978); Jaillet and Odoni (1988); Dror, Laporte, and Louveaux (1993); Mendoza, Castanier, Guéret, Medaglia, and Velasco (2010)), VRP with Stochastic Customers (Jézéquel (1985); Jaillet (1987); and Bertsimas (1992)), VRP with Stochastic Customers and Demands (Jézéquel (1985); Gendreau, Laporte, and Séguin (1996)), VRP with Stochastic Travel Time (Lambert, Laporte, \& Louveaux (1992)), VRP with Stochastic Demand and Travel Time (Cook \& Russell (1978)), VRP with Stochastic Travel Time and Service Time (Li, Tian, \& Leung (2010)).

\subsection{Energy Minimizing Vehicle Routing Problem}

The Energy Minimizing Vehicle Routing Problem (EMVRP) integrates the cost of vehicle energy consumption into the routing cost of the classic vehicle routing problem. Therefore, not only the energy consumption is minimized, but also the decrease in petroleum-based fuel consumption reduces the greenhouse gas emissions. One of the important components of the EMVRP is the energy consumption model used to estimate the vehicle energy requirements for routing. A more accurate energy consumption model results in a more realistic estimation of the vehicle energy requirements. The EMVRPs studied in the literature vary from each other in terms of the factors they consider in their energy consumption models. According to the report by the US Department of Energy (2008), travel speed, vehicle load and transportation distance are among the significant factors affecting the vehicle fuel consumption. Moreover, the results of the studies by Ardekani, 
Hauer, and Jamei (1996), Bigazzi and Bertini (2009), Demir et al. (2011) and Alwakiel (2011) show that, vehicle characteristics, environment and traffic conditions, and driver behavior are significant contributors to vehicle energy consumption. Most of the used models in the EMVRP studies concentrate on vehicle, traffic, and environmental aspects, and do not capture driver related factors which are relatively difficult to measure.

The EMVRP was first introduced by Kara et al. in 2007. They defined an EMVRP as a Capacitated VRP with the objective of minimizing the routing cost in the form of a weighted load function, defined as the product of total vehicle load and arc length. To examine the efficiency of the developed model in finding the optimal routes with minimum energy consumption, they compared the result of the defined model with a distance minimizing capacitated VRP on two different networks. The result of their study showed that the routes found by the EMVRP require less energy compared to those found in the distance minimizing CVRP. Another study focused on vehicle load in determining energy consumption was done by Xiao et al. in 2012. In this study the authors modeled fuel consumption as a linear function of vehicle load and distance. It was concluded that the shortest distance may not be the optimal solution for the purpose of lowering fuel consumption because distance and vehicle load contribute to the total fuel consumption jointly.

While these studies mainly focus on vehicle load in modeling the vehicle energy consumption, there are a number of other studies that consider speed as another contributing factor to energy consumption besides distance and load. Eglese and Black 
(2010) showed that speed is a more important factor than distance when estimating fuel consumption and emissions. Kuo (2010) solved the Time-Dependent VRP that aimed to minimize the fuel consumption as a function of vehicle speed varying over different time of day. The study findings showed that the proposed method provided a $24.61 \%$ improvement in fuel consumption over the method based on minimizing transportation time and a $22.69 \%$ improvement over the method based on minimizing transportation distances. In another study by Maden (2010) it was concluded that the standard TimeDependent VRP with the objective of minimizing total travel time results in a saving in fuel consumption as the model avoids congested links in order to minimize the travel time. Their study results showed an average of $7 \%$ reduction in the vehicles fuel consumption. Jovicic et al. (2010) investigations showed that in the City of Kragujevac in Serbia, a reduction of up to $20 \%$ can be achieved in energy costs and the associated emissions if the effect of vehicle speed on the vehicle energy consumption is accounted for in the routing of the municipal waste collection.

Contrary to these studies in which the vehicle speed is given, Bektas and Laporte (2011) solved a capacitated VRP with Time Windows with the speed on each arc as a primary decision variable in order to find the optimal speed for vehicle movements along each arc such that the total energy consumption is minimized. The energy consumption in this study was formulated as a function of vehicle load and travel speed. In another research done by Demir, Bektas, and Laporte (2013) the tradeoffs between fuel consumption and driving time were investigated. They showed that trucking companies need not compromise greatly in terms of driving time in order to achieve a significant 
reduction in fuel consumption and $\mathrm{CO} 2$ emissions. They also argued that the converse of this insight holds too and considerable reductions in driving time are achievable if one is willing to increase fuel consumption only slightly.

Tavares et al. (2008) looked at an EMVRP optimizing the routing cost of waste transportation by taking into account the energy consumption as a function of road angle besides vehicle load. Their findings indicated that optimizing fuel consumption can yield savings of up to $52 \%$ in fuel when compared with minimizing distance. In another paper by Tavares et al. (2009) the routing of municipal solid waste collection fleet was optimized by minimizing the fuel consumption using 3D GIS modeling with road gradient playing the main role in energy requirement considerations. The results of this study suggested that the proposed methodology reduced traveled distance and fuel consumption by $29 \%$ and $16 \%$ respectively. The most comprehensive energy model was used in Demir et al. (2011) study where they compared several energy consumption models, and revealed other relevant contributing factors such as driver acceleration behavior, engine type and size, vehicle design, besides road gradient, speed and vehicle load.

\section{$\underline{\text { 2.3 Pollution Routing Problem (PRP) }}$}

While Energy Minimizing VRP yields to less routing emission by minimizing vehicle energy consumption, Pollution Routing Problem (PRP) tries to minimize the emission by incorporating emission cost directly in the objective function of VRP. 
Although classic VRP with the objective of minimizing the total distance traveled will result in less emission (Maden et al. (2010)), there are other factors that affect the vehicle generated pollution besides distance. If these factors are accounted for, the efficiency of the VRP in reducing the emission increases. Literature studies show that travel speed is one of the significant factors affecting the vehicle generated emission. Sibhi and Egles (2007) argued that by avoiding congestion in vehicle routing, although the travel distance might increase, the routing emission will significantly decrease. The result of their Time-Dependent VRP model with the objective of minimizing travel time showed significant reduction of $\mathrm{CO} 2$ emissions after planning routes according to the time-varying speeds. In a paper by Palmer (2007) the effects of speed on CO2 emission was examined through a developed integrated routing and carbon dioxide emissions model calculating the amount of $\mathrm{CO} 2$ emission, travel time and distance on the journey. Different congestion scenarios were considered and the results showed that about $5 \%$ of reduction of $\mathrm{CO} 2$ emissions could be achieved by accounting for speed variations in vehicle routing. Similar results were found in the PhD thesis of Qian (2012) on the vehicle emission minimization in VRP with time-varying speeds. The objective of this dissertation was to formulate a Time-Dependent VRP model that generates routes and schedules for a fleet of heavy goods vehicles such that the emissions in a network with Time-Dependent travel speed is minimized. The developed algorithm was tested on a London case study and the result suggested a 6-7\% savings in fuel. Considering the important role of speed in pollutant emission of vehicles Fagerholt et al. (2010) tried to minimize the pollutant emissions on a set of fixed shipping routes by optimizing speed. 
While in the above mentioned studies the objective of the developed PRP models were to minimize the environmental effects solely, some other studies sought to formulate a VRP model with more comprehensive objective functions minimizing economic and environmental costs jointly such that the cost efficiency objectives and green criteria are met simultaneously. One of these studies was done by Bektas and Laporte (2011) in which they proposed a Pollution Routing Problem with or without time windows and developed a comprehensive objective function that aimed to minimize the cost of carbon emissions and the operational costs of drivers and fuel consumption jointly. The model was solved to find the optimal routes with optimal speed on each arc on the route. Although the computational results showed the model's efficiency in reducing fuel consumption and emission, the model did not represent real world situations as it was designed for a freeflow speed of $40 \mathrm{~km} / \mathrm{h}$. Following up this research, Demir, Bektas, Laporte (2012) proposed an extended Adaptive Large Neighborhood Search (ANLS) for PRP in order to enhance the computational efficiency for medium or large scale PRP. In another study later in 2013, they developed a bi-objective PRP model to find the optimal speed and route for vehicles such that the total emission and travel time are minimized. The logic behind using a bi-objective model was the conflict in trying to minimize fuel consumption by reducing speed while trying to minimize driving time as well. The result of their study showed that reducing the emission can be achieved by increasing the total duration of routes. It was seen that a $9.7 \%$ increase in driving time led to a $27 \%$ saving in energy requirements while reduction in driving time from 23.21 hours to 21.16 hours (about 8.8\%) increased $\mathrm{CO} 2 \mathrm{e}$ emissions by about $37.7 \%$. 


\subsection{Alternative Fuel Vehicle Routing Problem}

The Alternative Fuel VRP (AFVRP) is a type of GVRP which not only aims to minimize the vehicles' energy consumption but also tries to address the limitations in the use of AFVs such as range limitations. The AFVRP is closely related to the classical distanceconstrained VRP (Laporte et al., 1985), however, in AFVRP there is a possibility of extending the vehicle's distance limitation by visiting charging stations. Therefore, existing solutions to distance-constrained VRP could not be applied to AFVRP. Another problem that is closely related to the AFVRP is the multi-depot vehicle routing problem with inter-depot facilities described by Bard et al. (1998). This problem considers intermediate depots at which vehicles can be reloaded to serve customer demands.

In the literature, relatively few studies have been published on alternative fuel vehicle optimization problems. The paper by Erdogan and Miller-hooks (2012) was one of the pioneers in AFVRP studies. In this study an AFVRP model was developed to optimally route a fleet of un-capacitated AFVs with a limited driving range and the possibility of refueling at dedicated stations having unlimited capacity. Two heuristics were proposed to find the tours with minimum total distance, while eliminating the risk of running out of fuel. It was assumed that the vehicles are fully charged upon each visit to charging stations with a constant charging time. In 2013, Barco et al. (2012) expanded Erdogan and Miller-hooks study by considering more realistic assumptions such as vehicle load capacity. They tried to find optimal routes for a set of homogeneous capacitated electric airport shuttles by minimizing total energy consumption, recharging and battery degradation costs. Scheduling of charges was coordinated with routing to guarantee a 
reliable operation serving the demand of customers within their time windows while accounting for the variation of the energy cost during the peak and non-peak hours of the day. In a study done by Schneider et al. in 2014 a different variant of capacitated AFVRP with customer time windows was solved in which the optimal number of required vehicles had to be found as well as the minimum total distance tours using a hierarchical objective function. All available electric vehicles were assumed to be homogeneous and the charging time assumed to vary depending on the state of the battery upon arrival to charging stations. Felipe et al. (2014) extended Erdogan and Miller-Hooks study in an alternative way by including realistic considerations such as the possibility of performing a partial recharge at a station and the availability of different charging technologies, implying different recharging time and cost (Slow, Fast and wireless). The variability in charging policies used in previous studies motivated Desaulniers et al. in 2014 to investigate the effect of these different charging policies on the total cost of a fleet of electric delivery trucks routing to distribute goods to customers with soft service time windows. The result showed that allowing multiple and partial charges along the route for each electric truck helps to reduce the routing cost and the number of employed vehicles in comparison to the variants with single and full charges. The results from Bruglieri et al. study in 2015 showed the same findings. They proposed a Mixed Integer Linear Programming formulation of the EVRP problem with Time Windows to minimize the total travel, waiting and recharging time plus the number of the employed EVs assuming partial charging multiple stops is allowed. Their findings showed that partial charging policy outperforms the full charging one in terms of recharging, waiting and travel time. 
While all these studies were focusing on a homogeneous fleet of AFVs, Hiermann et al. in 2014 expanded the AFVRP with Time Windows by considering a mixed fleet of fixed size electric vehicles with different battery capacities, load capacities and purchase costs. The charging policy considered in this study was the single and full charge in each route. Considering the fact that most companies do not operate pure EV fleets and are gradually introducing ECVs into their existing internal combustion engine vehicle fleet, Sashi et al. (2015) tried to model and solve an EVRP with a mixed fleet of heterogeneous EVs and homogeneous Combustion engine commercial vehicles. The objective of this study was to find the minimum cost routes while accounting for limited range of EVs. It was assumed that the charging cost is dependent on the time of a day and specific working hours were assumed for charging stations. Moreover it was assumed that EVs can only charge at charging stations with compatible charging techniques with partial charging allowed. A greener version of Mixed Fleet EVRP was studied by Goekea and Schneider (2015). The developed model in their study was aimed to minimize vehicle energy consumption cost. The vehicle energy requirements were modeled as a function of travel speed, gradient and cargo load, and contrary to Sashi's study only full charge of the EVs was allowed upon each visit to charging stations. The result of their study showed that consideration of the vehicle load in EV battery consumption estimation model strongly improves the quality of the generated routing solutions. Moreover, it was found that a large number of solutions that are generated without load estimates are actually infeasible due to battery capacity. 


\section{$\underline{2.5 \text { Research Gap }}$}

In this dissertation, a more comprehensive GVRP is defined to address the gaps in literature. First of all, due to the sensitivity of ECV's driving range to the energy consumption rate a Time-Dependent GVRP is introduced. By accounting for time dependency of travel time along the arcs in the network, recurring congestion is taken into account and a more realistic energy requirement is estimated while routing vehicles. Moreover, while all the studies in the literature only focus on the limitations of ECV adoption, in this dissertation a new problem is defined where there are limitations in adoption of both types of the vehicles. These limitations are limited driving range and high acquisition cost for ECVs and LEZ penalty and emission cap for ICCVs. Contrary to previous studies a heterogeneous fleet of ECVs and ICCVs with different battery and load capacities are considered in the problem. The route plans are not determined for a prespecified number of vehicles. It is assumed that the number of vehicles is not predefined and the optimal number of vehicles of each type is to be found. 


\section{Chapter 3}

\section{MODEL FORMULATION - GVRP}

In this Chapter a mathematical model is formulated for the problem explained in section 1.2 where travel time on arcs is not dependent on time of day and is constant. The problem is formulated as a mixed integer linear programming problem on a network. First, the problem properties are described completely. Then, the assumptions and limitations of the model are given. In the third section, the mathematical formulation of the problem is explained which includes detailed explanations of the notations and variables used in the model, the objective function, and the constraints. At last, the developed model is solved on a set of small size network problems. The chapter is summarized in the last section.

\subsection{Characteristics of the Problem}

\subsubsection{Network}

The GVRP is formulated on a complete directed graph $G=\left(V_{0, N+1}^{\prime}, A\right)$. Vertices $O$ and $N+1$ denote instances of depot. $V^{\prime}$ is the union of the set of demand nodes $(V=\{1,2, . ., N\})$ and the set of charging station visit nodes $\left(F^{\prime}\right)$, which represent the set of visits to vertices in the set of charging stations, $F$. All vehicle routes start from node 0 and end at node $N+l$. The set of arcs is given by $A=\left\{(i, j) \mid(i, j) \in V_{0, N+1}^{\prime}, i \neq j\right\}$. Each arc is described by travel time, $t t_{i j}$, travel speed, $V_{i j}$, and average acceleration rate, $a_{i j}$. 


\subsubsection{Demand Nodes and charging nodes}

A nonnegative demand, $q_{i}$, and a nonnegative service time, $s_{i}$ is associated with each demand node in set $V$. There is also a service time window $\left[e_{i}, l_{i}\right]$ for each demand node within which the service to a customer has to start. Each charging station has a nonnegative service time and there is no specific time window for charging stations operating hours.

\subsubsection{Commercial Vehicle Types}

A mixed fleet of heterogeneous electric commercial vehicles, ECVs, with different battery and loading capacity, and heterogeneous Internal Combustion Engine Vehicles, ICCVs, with different loading capacities is considered to be available in this study. The number of vehicles is not predefined and it is one of the objectives of the problem to find the optimal number of vehicles of each type to be used to serve the demand.

\subsubsection{Energy Consumption of Electric Commercial Vehicles}

One of the challenges in using commercial electric vehicles for distribution operations is the limited autonomy of these vehicles. Due to this limitation in driving ranges, GVRPs should provide the possibility of enroute recharging at available charging stations while providing optimal routes for these vehicles. The necessity to visit a charging station depends on the available battery level of the ECVs which itself depends on the battery consumption during the route. As it was described in section 2.2, energy consumption of vehicles depends on travel speed, driver acceleration behavior, vehicular characteristics such as vehicle age, and mass, and the road geometry and environmental conditions. In 
the literature, most of the studies on EVRP have modeled the energy consumption of EVs as a linear function of the traveled distance or speed. While these two factors are among the significant contributors to vehicle energy consumption, accounting for the other factors enhance the accuracy of the energy consumption estimations and leads to more efficient routing plans compatible with real-world situations. In this study, the model developed in Goeke and Schneider's (2015) study is used to calculate the battery energy consumption of ECVs as this model accounts for a more comprehensive contributing factors to vehicle energy requirements. The energy consumption of EVs is estimated in three steps as shown in Figure 3.1.

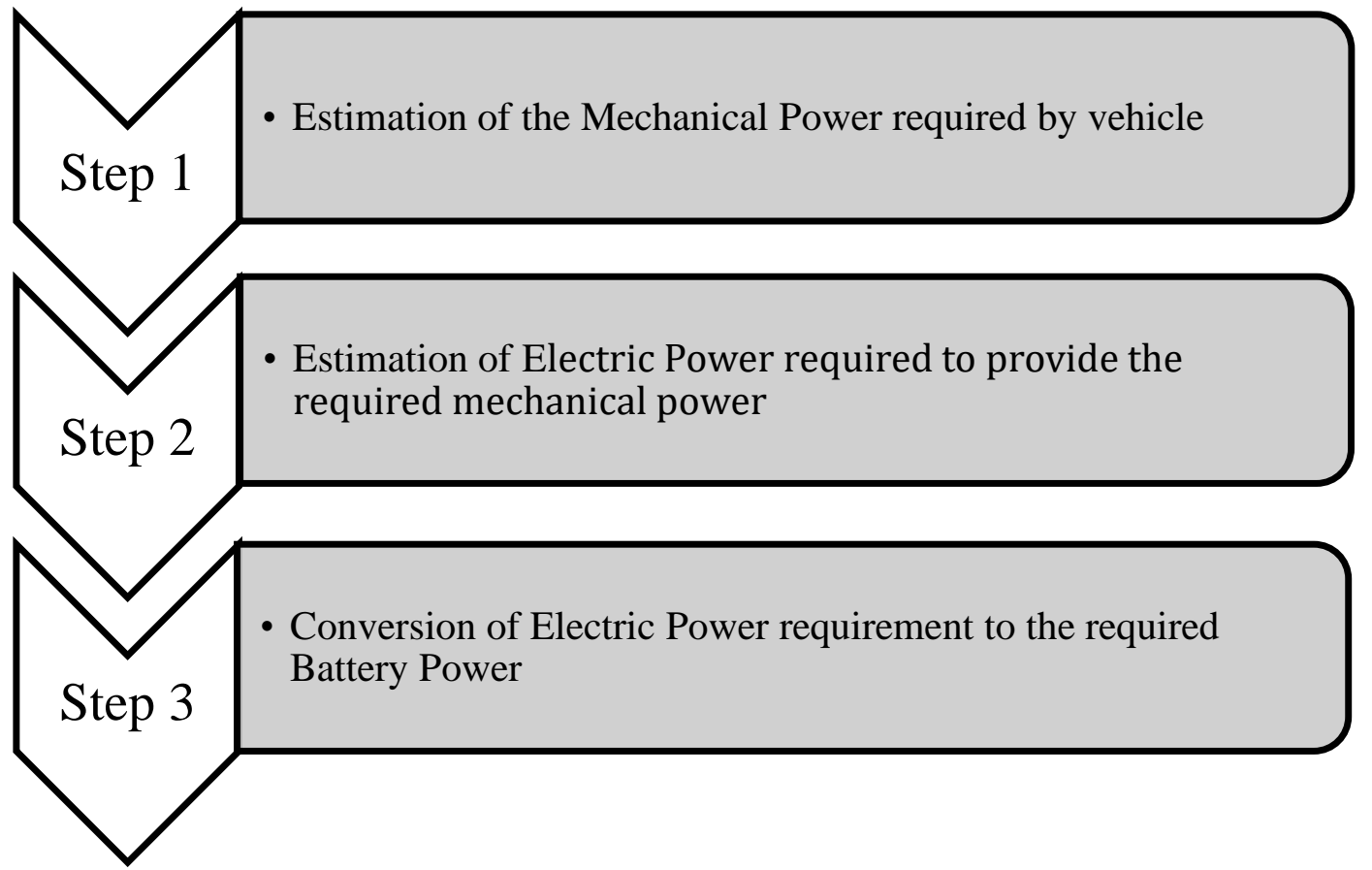

Figure 3.1 The Procedure in Estimating ECV Energy Consumption 
First, the mechanical power, $P_{M}$, required by the vehicle to overcome rolling resistance, aerodynamic resistance and gravitational force is estimated using the model presented in Bektas and Laporte study (2011).

$$
\begin{aligned}
& P_{M}=\left(m \cdot a+\frac{1}{2} \cdot c_{d} \cdot \rho \cdot A \cdot v^{2}+m \cdot g \cdot \sin z+c_{r} \cdot m \cdot g \cdot \cos z\right) \cdot v \\
& m=W+U \\
& P_{M}=(W+U) \cdot a+\frac{1}{2} \cdot c_{d} \cdot \rho \cdot A \cdot v^{3}+(W+U) \cdot v \cdot g \cdot \sin z+c_{r} \cdot(W+U) \cdot v \cdot g \cdot \cos z(3 \cdot 3)
\end{aligned}
$$

Where,

- $\quad m=$ Vehicle mass as the sum of vehicle weight $(W)$ and vehicle load $(U)$

- $\quad a=$ Vehicle acceleration

- $C_{d}=$ Aerodynamic drag coefficient

- $\rho=$ Air Density

- $A=$ Frontal Area of the vehicle

- $C_{r}=$ Rolling friction coefficient

- $\quad V=$ Vehicle Speed

- $\quad z=$ Road gradient

As it can be seen, using this formula enables the energy consumption model to capture the effect of speed, acceleration, vehicle load and road gradient in finding optimal routes with minimum required energy. In the next step the electric power, $P_{E}$, required to provide 
the mechanical power $\left(P_{M}\right)$ is estimated using the relationship found in Goeke and Schneider's study (2015).

$P_{E}=\phi P_{M}$

Where, $\phi$, is the regression coefficient found by fitting a homogeneous linear regression line with a y-axis intercept of zero to a set of observed $P_{M}$ and discharged electric energy data while the engine energy losses is accounted for. In the third and last step, the required electric power $\mathrm{P}_{\mathrm{E}}$ is converted to the amount of power that needed to be taken from the battery, $\mathrm{P}_{\mathrm{B}}$, which significantly depends on the efficiency of the battery (Van Keulen et al.; 2010).

$P_{B}=\varphi P_{\mathrm{E}}$

Where, $\varphi$ is the regression coefficient that describes the battery efficiency. Once the required battery electric power is estimated through these three steps, the battery energy consumption of an EV traveling on $\operatorname{arc}(i, j)$ with the travel time $t_{i j}$ can be estimated as:

$b_{i j}=P_{B} t t_{i j}=\varphi \phi P_{i j} t_{i j}$ 
Where, $P_{i j}$, is the vehicle's required mechanical power to traverse arc $(i, j)$ estimated by equation 3.1, and $b_{i j}$ is the battery electric energy consumption of EV for traveling arc $(i, j)$.

\subsubsection{Energy Consumption of Internal Combustion Commercial Vehicles}

To estimate the energy consumption of combustion engine vehicles, first, the vehicle required mechanical power, $P_{M}$, is estimated using equation 3.3. Then, the estimated $P_{M}$ is converted to fuel consumption rate, $F R$, using the model introduced by Barth (2005).

$F R=\frac{\xi}{K \cdot \psi}\left(K N D+\frac{P_{M}}{\eta \cdot \eta_{t f}}\right)$

Where,

- $\xi=$ Fuel-to air mass ratio

- $\quad k=$ Heating value of typical diesel fuel

- $K=$ Engine friction factor

- $\quad N=$ Engine speed

- $D=$ Engine displacement

- $\psi=$ Factor converting the fuel rate from grams per second to liters per second

- $\eta=$ Efficiency parameter for diesel engines

- $\eta_{t f}=$ Drive train efficiency 
Having calculated the fuel consumption rate, the total fuel consumption of an ICCV traversing an arc $(i, j)$ with travel time $t_{i j}$ can be calculated as:

$f_{i j}=F R_{i j} \cdot t_{i j}$

where, $F R_{i j}$ is the fuel consumption rate of the vehicle on $\operatorname{arc}(i, j)$.

\section{$\underline{\text { 3.1.6 Emission Model }}$}

The instantaneous engine-out Greenhouse Gas emission rate, $E$, in grams per second $(\mathrm{g} / \mathrm{s})$ is directly related to the fuel consumption rate, $F R$ (Bektas and Laporte, 2011). Therefore, the vehicle emission is estimated in gram per second $(\mathrm{g} / \mathrm{s})$ through the equation 3.7.

$E=\delta_{1} F R+\delta_{2}$,

where $\delta_{1}$ and $\delta_{2}$ are GHG-specific emission index parameters.

\section{$\underline{3.2 \text { Assumptions }}$}

\subsubsection{Customer Service Time Window}

The service time windows associated with demand points in the network are assumed to be soft time windows as they are more realistic and more flexible than hard time windows. There is a time penalty associated with early or delayed services meaning that if the demands are not served on time, a delay or waiting penalty is imposed. The time penalty 
coefficients can be adjusted to reflect the tradeoffs between the fixed cost of using an additional vehicle to provide an on-time service to all customers or pay the time penalties while using less number of vehicles.

\subsubsection{Charging Station Type}

The charging stations are assumed to be of battery swapping type meaning that once an ECV reaches a charging station its battery is swapped with a fully charged battery. Therefore, the charging time is assumed to be the same for all types of ECVs at all charging station locations.

Battery swapping stations have been in practice for Tesla passenger vehicles since 2014. Findings of Chang (2010) and Kim (2012) studies suggest that battery swapping stations have advantages for logistics companies. First of all, by using battery swapping stations the whole charging process can take up to 10 minutes which eliminates the existing problem of wasting a considerable amount of time at charging stations and makes the electric vehicles on par with combustion engine vehicles. On the other hand, charging the depleted batteries during the night or any time of the day that demand and price of electricity is lower helps to significantly reduce the operation cost of electric vehicles (United Nations Environment Program (2010)). Moreover, since the logistics company owns the batteries it swaps out, the sticker price of ECVs that can use its network would be cheaper. Therefore, battery swapping stations benefit logistics companies by facilitating the charging process and use of electric commercial vehicles while lowering the purchase and daily operational cost of these vehicles. 


\subsubsection{Vehicle energy consumption}

The electric energy and fuel consumption of ECVs and ICCVs traveling an arc $(i, j)$ are respectively determined by equations explained in section 3.1.4 and 3.1.5. In these equations, the travel speed is assumed to be constant and known for each arc of the network. Also an average acceleration rate is associated with each arc and it is assumed to be constant and the same for all vehicles types. The road gradient is assumed to be 0 due to the negligible altitude variations in DC metropolitan area. However, the effect of altitude variations on energy consumption can be accounted for by the model, when applicable, by inserting the road elevation as an input to both ECV and ICCV energy consumption models described respectively in sections 3.1.4 and 3.1.5. Although the battery of ECVs can regenerate electricity on downhill, the model used in this dissertation can only account for electric energy consumed on uphill and cannot be used to estimate the regenerated electric energy by ECV batteries while driving on downhill.

\subsubsection{Government Incentives and Regulations}

It is assumed that there is a financial incentive by government subsidizing the purchase of ECVs. Moreover, it is assumed that there is a number of Low Emission Zones (LEZ) in the network operating 24 hours a day and 365 days of a year such that combustion engine commercial vehicles are required to pay a daily charge to drive within these zones. The daily charges are assumed to be exclusive to each LEZ and paying the charge for one LEZ does not give the permission to ICCVs to enter all other LEZs in the network. The LEZs are characterized by the set of demand nodes located in these zones. Figure 3.2 shows an 
example of the daily charges required to be paid by different vehicle types to enter London Low Emission Zone.

\begin{tabular}{|c|c|c|}
\hline Vehicle & Weight & Daily charge \\
\hline $\begin{array}{l}\text { Larger vans - Motorised horseboxes } \\
4 \times 4 \text { light utility vehicles } \\
\text { Pick-ups } \\
\text { Other specialist vehicles }\end{array}$ & 1.205 tonnes unladen & $£ 100$ \\
\hline Motor caravans - Ambulances & $2.5-3.5$ tonnes gross vehicle weight & $£ 100$ \\
\hline Minibuses (with more than 8 passenger seats) & 5 tonnes or less gross vehicle weight & $£ 100$ \\
\hline $\begin{array}{l}\text { Lorries - Goods vehicles } \\
\text { Motor caravans } \\
\text { Motorised horseboxes } \\
\text { Breakdown and recovery vehicles } \\
\text { Snow ploughs } \\
\text { Gritters } \\
\text { Refuse collection vehicles } \\
\text { Road sweepers } \\
\text { Concrete mixers } \\
\text { Tippers } \\
\text { Fire engines } \\
\text { Removals lorries } \\
\text { Other specialist vehicles }\end{array}$ & More than 3.5 tonnes gross vehicle weight & $£ 200$ \\
\hline $\begin{array}{l}\text { Buses } \\
\text { Coaches (with more than } 8 \text { passenger seats) }\end{array}$ & More than 5 tonnes gross vehicle weight & $£ 200$ \\
\hline
\end{tabular}

Figure 3.2 London LEZ Daily Charges - Source: https://tfl.gov.uk/modes/driving/lowemission-zone/make-a-payment

Another assumption is the existence of emission cap and trade regulation imposed by the government that limits the amount of pollution that the company can produce in a year. This amount is converted to an average emission per day of operation and is treated as a threshold for routing emission. If the amount of emission produced in a daily routing 
operation is more than this limit, the company is charged for each additional gram of emitted pollutant. On the other hand, it is assumed that any extra emission permission that is not used in a daily operation can be sold to other companies at the end of each operation day.

\subsection{Mathematical Formulation}

The GVRP of interest is formulated as a mixed integer linear programming problem based on the above assumptions. In the following subsections, the notations, coefficients, and variables used in the model are introduced and the objective function and constraints are explained.

\subsubsection{Notation and Variables}

\section{$\underline{\text { Data Sets }}$}

$V \quad$ Set of demand nodes

$F^{\prime} \quad$ Set of Charging Station visit nodes, dummy vertices of the set of charging stations $F$

$V^{\prime} \quad V \cup F^{\prime}$

$V_{0}, V_{N+1}$ Instances of depot

$V_{0, N+1} \quad V \cup V_{0} \cup V_{N+1}$

$V_{0, N+1}^{\prime} \quad V^{\prime} \cup V_{0} \cup V_{N+1}$

$L E Z_{l} \quad$ Set of demand nodes in the low emission zone $l \in\{1,2, \ldots, L\}$

$C_{C V} \quad$ Set of ICCV types 
$C_{E V} \quad$ Set of ECV types

\section{$\underline{\text { Constants }}$}

$q_{i} \quad$ Nonnegative demand of node $i \in V$

$e_{i} \quad$ Earliest service time of node $i \in V$

$l_{i} \quad$ Latest service time of node $i \in V$

$S_{i} \quad$ Service time of node $i \in V^{\prime}$

$F C_{c}^{E V} \quad$ Fixed cost of EV type $c \in C_{E V}\left(\frac{\$}{d a y}\right)$

$F C_{c}^{C V} \quad$ Fixed cost of ICCV type $c \in C_{C V}\left(\frac{\$}{d a y}\right)$

$Q_{c}^{E V} \quad$ Loading capacity of EV type $c \in C_{E V}$

$Q_{C}^{C V} \quad$ Loading capacity of ICCV type $c \in C_{C V}$

$W_{c}^{E V} \quad$ Weight of empty EV of type $c \in C_{E V}$

$W_{c}^{C V} \quad$ Weight of empty ICCV of type $c \in C_{C V}$

$B C_{c}^{E V} \quad$ Battery Capacity of EV type $c \in C_{E V}$

$C^{E} \quad$ Cost of Electricity $\left(\frac{\$}{k w}\right)$

$C^{F} \quad$ Cost of Fuel $\left(\frac{\$}{\text { liter }}\right)$

$C^{L} \quad$ Cost of Labor

$m_{c}^{E V} \quad$ Maximum number of available EV of type $c \in C_{E V}$

$m_{c}^{C V} \quad$ Maximum number of available ICCV of type $c \in C_{E V}$

$v_{i j} \quad$ Travel speed on $\operatorname{arc}(i, j) \mid(i, j) \in V_{0, N+1}^{\prime}, i \neq j$ 


$\begin{array}{ll}a_{i j} & \text { Average acceleration rate on } \operatorname{arc}(i, j) \mid(i, j) \in V_{0, N+1}^{\prime}, i \neq j \\ t t_{i j} & \text { Travel time on arc }(i, j) \mid(i, j) \in V_{0, N+1}^{\prime}, i \neq j \\ W P & \text { Waiting time penalty } \\ D P & \text { Delay time penalty } \\ P & \text { LEZ daily penalty } \\ e^{C A P} & \text { Emission Cap } \\ P_{C a r b} & \text { Price of carbon }\left(\frac{\$}{\text { gram }}\right) \\ P B & \text { Battery Cycle Cost } \\ g h g & \text { Greenhouse Gas emissions per liter of fuel }\left(\frac{\mathrm{Kg}}{\text { liter }}\right) \\ T_{0}, T & \text { Start and End time of delivery operations }\end{array}$

\section{Decision Variables}


$U_{i j c k}^{C V} \quad$ Load carried by combustion engine vehicle $k$ of type $c$ from node $i$ to node $j$

$R_{\text {ick }} \quad$ Remaining battery of electric vehicle $k$ of type $c$ upon arrival at node $i$

$w_{i c k}^{E V} \quad$ Waiting time of electric vehicle $k$ of type $c$ at node $i$

$w_{i c k}^{C V} \quad$ Waiting time of combustion engine vehicle $k$ of type $c$ at node $i$

$d_{i c k}^{E V} \quad$ Delayed time of electric vehicle $k$ of type $c$ at node $i$

$d_{i c k}^{C V} \quad$ Delayed time of combustion engine vehicle $k$ of type $c$ at node $i$

\subsubsection{Objective Function}

The objective of this problem is to minimize the total vehicle purchase cost, routing fuel and electric energy consumption cost, labor cost and the total LEZ, service time, and carbon penalty cost.

Minimize:

$$
\begin{aligned}
& \sum_{c \in C}^{E V} \sum_{k \in S_{C}^{E V}} \sum_{j \in V} F_{c}^{E V} \cdot x_{0 j c k}^{E V}+\sum_{c \in C} C V \sum_{k \in S_{C}^{C V}} \sum_{j \in V} F_{C}^{C V} \cdot x_{0 j c k}^{C V}+ \\
& \sum_{c \in C} C V \sum_{k \in S_{C}^{C V}} \sum_{i \in V_{0}} \sum_{j \in V_{n+1}} C^{F}\left(\alpha_{i j} \cdot w_{c}^{C V}+\beta_{i j}\right) \cdot t_{i j} \cdot x_{i j c k}^{C V}+ \\
& \sum_{c \in C} C V \sum_{k \in S_{C}^{C V}} \sum_{i \in V_{0}} \sum_{j \in V_{n+1}} C^{F}\left(\propto_{i j} \cdot U_{i j c k}^{C V}\right) . t_{i j}+ \\
& \sum_{c \in C^{E V}} \sum_{k \in S_{C}^{E V}} \sum_{i \in V^{\prime} 0} \sum_{j \in V^{\prime} n+1} C^{E}\left(\alpha^{\prime}{ }_{i j} \cdot w_{c}^{E V}+\beta_{i j}\right) \cdot t_{i j} \cdot x_{i j c k}^{E V}+ \\
& \sum_{c \in C}^{E V} \sum_{k \in S_{c}^{E V}} \sum_{i \in V^{\prime} 0} \sum_{j \in V^{\prime} n+1} C^{E}\left(\alpha^{\prime}{ }_{i j} \cdot U_{i j c k}^{E V}\right) \cdot t_{i j}+ \\
& P \sum_{c \in C} C V \sum_{k \in S_{c}^{C V}} \sum_{l \in L} y_{k l}^{C V}+W P\left(\sum_{i \epsilon V} \sum_{c \epsilon C}^{E V} \sum_{k \in S_{c}^{E V}} w_{i c k}^{E V}+\right.
\end{aligned}
$$




$$
\begin{aligned}
& \left.\sum_{i \epsilon V} \sum_{c \epsilon C^{C V}} \sum_{k \epsilon S_{c}^{C V}} w_{i c k}^{C V}\right)+D P\left(\sum_{i \epsilon V} \sum_{c \epsilon C^{E V}} \sum_{k \epsilon S_{c}^{E V}} d_{i c k}^{E V}+\right. \\
& \left.\sum_{i \epsilon V} \sum_{c \epsilon C^{C V}} \sum_{k \epsilon S_{c}^{C V}} d_{i c k}^{C V}\right)-P_{c a r b}\left(e^{C A P}-g h g\left[\left(\propto_{i j} \cdot w_{c}^{C V}+\right.\right.\right. \\
& \left.\left.\left.\beta_{i j}\right) \cdot t_{i j} \cdot x_{i j c k}^{C V}+\left(\propto_{i j} \cdot U_{i j c k}^{C V}\right) \cdot t_{i j}\right]\right)+ \\
& C^{L}\left[\sum_{i \epsilon V^{\prime}{ }_{0, N+1}} \sum_{j \epsilon V^{\prime}, N+1} \sum_{c \epsilon C^{E V}} \sum_{k \epsilon S_{c}^{E V}} x_{i j c k}^{E V} \cdot\left(S_{i}+t_{i j}\right)+\right. \\
& \left.\sum_{i \epsilon V_{0, N+1}} \sum_{j \epsilon V_{0, N+1}} \sum_{c \epsilon C} C V \sum_{k \epsilon S_{c}^{C V}} x_{i j c k}^{C V} \cdot\left(S_{i}+t_{i j}\right)\right]+ \\
& P B\left(\sum_{i \epsilon V_{0}} \sum_{j \epsilon F^{\prime}} \sum_{c \epsilon C^{E V}} \sum_{k \epsilon S_{c}^{E V}} x_{i j c k}^{E V}\right)
\end{aligned}
$$

The first row of the objective function is the vehicle purchase cost. As it was mentioned before, in this problem the number of vehicles is not fixed. In fact, the objective function tries to minimize the number of required vehicles to serve the customer demands with the available vehicle types. Assuming that the battery life cycle of the electric vehicles is 5 years and the vehicles' residual value is at $20 \%$ of their purchase cost, the equations below are used to convert the vehicle purchase cost of ICCV and ECV to dollar per day for a planning horizon of 5 years. In this equation the future residual value of vehicle is discounted back at $2 \%$ continuously compounded annual rate that is in line with inflation. A continuously compounded rate has been used to reflect daily compounded interest associated with the opportunity cost of capital at $2 \%$.

$\frac{F C}{D a y}=V P C-G P S-V R V \times e^{-r T}$ 
Where,

- $\quad V P C=$ Vehicle Purchase Cost

- $\quad$ GPS = Government Purchase Subsidy

- $\quad V R V=$ Vehicle Residual Value

- $\quad T=$ Planning horizon in years

- $\quad r=$ Annual interest rate

The fuel energy consumed by ICCVs is minimized in the second and third row of the objective function followed by the electric energy consumption of ECVs minimized in the fourth and fifth rows. The natural integer programming formulation of the GVRP developed in this study is nonlinear as the energy function contains the decision variable $U_{i j}$ which multiplied with $x_{i j}$, makes the objective function nonlinear. The procedure below is taken to linearize this nonlinear term. The vehicle energy requirement function shown in equation 3.3 is decomposed into two parts. Therefore, if an arc $(i, j)$ is traversed by any of the available vehicles, the first part is multiplied by $x_{i j}$ to estimate the energy requirements of the vehicle due to its body weight, and the second part estimates the vehicle energy requirements due to its load. $U_{i j}$ for each vehicle is forced to zero if the arc $(i, j)$ is not used by the vehicle $\left(x_{i j}=0\right)$ through a set of constraints explained in the next section.

$P_{M}^{C V}=\propto_{i j} \cdot\left(W+U_{i j}\right)+\beta_{i j}=\left(\propto_{i j} \cdot W+\beta_{i j}\right)+\propto_{i j} \cdot U_{i j}$ 
$P_{M}^{E V}=\propto^{\prime}{ }_{i j} \cdot\left(W+U_{i j}\right)+\beta_{i j}^{\prime}=\left(\propto^{\prime}{ }_{i j} \cdot W+\beta^{\prime}{ }_{i j}\right)+\propto^{\prime}{ }_{i j} \cdot U_{i j}$

Where,

$\propto_{i j}=v_{i j} \cdot a_{i j}+v_{i j} \cdot g \cdot \sin z+c_{r} \cdot v_{i j} \cdot g \cdot \cos z$

$\beta_{i j}=\frac{1}{2} \cdot c_{d} \cdot \rho \cdot A \cdot v_{i j}^{3}$

$\propto^{\prime}{ }_{i j}=\varphi \phi \propto_{i j}$

$\beta^{\prime}{ }_{i j}=\varphi \phi \beta_{i j}$

- $\quad W=$ Vehicle body weight

- $U_{i j}=$ Vehicle load ove arc $(i, j)$

- $a_{i j}=$ average acceleration rate over $\operatorname{arc}(i, j)$

- $v_{i j}=$ Average travel speed over arc $(i, j)$

- $\quad C_{d}=$ aerodynamic drag coefficient

- $\rho=$ Air Density

- $A=$ Frontal Area of the vehicle

- $C_{r}=$ Rolling friction coefficient

- $\quad z=$ Road gradient

The sixth row of objective function minimizes the LEZ penalty cost imposed if any ICCV enters any LEZ zone in the network. It accounts for the tradeoffs between the low 
emission zone penalty cost imposed on ICCVs and the fixed cost of using an additional electric vehicle to serve demands in LEZ zones.

The user inconvenience cost is minimized in the seventh and eights rows of the objective function in the form of waiting or delayed service cost penalties. All demand nodes have desired service time windows. Whenever the service is delayed or is started earlier than the customer's desired time, a service time penalty is incurred.

The ninth row tries to minimize the emission cost by decreasing the cost of emission produced more than the limit or by increasing the amount of extra emission credit to be sold to other companies.

The labor cost is minimized in the tenth and eleventh rows. The final row minimizes the battery degradation cost which is calculated as the multiplication of the battery cycle cost by the number of times the battery of ECV is replaced at a charging station. The battery cycle cost is estimated by dividing the cost of battery by the number of times it can be fully charged in its life cycle.

\section{$\underline{\text { 3.3.3 Constraints }}$}

In this subsection, the problem constraints are provided, with a brief explanation for each. Constraint 3.18 ensures that each demand node has exactly one successor.

$$
\sum_{c \in C_{E V}} \sum_{k \in K_{E V}} \sum_{j \in V^{\prime} N+1} x_{i j c k}^{E V}+\sum_{c \in C_{C V}} \sum_{k \in K_{C V}} \sum_{j \in V_{N+1}} x_{i j c k}^{C V}=1 \quad \forall i \in V, i \neq j
$$


Constraints 3.19 and 3.20 guarantee that for each node in the network the number of incoming arcs is equal to the number of outgoing arcs for each vehicle type.

$\sum_{j \in V^{\prime} N+1} x_{i j c k}^{E V}-\sum_{j \in V^{\prime}{ }_{0}} x_{j i c k}^{E V}=0 \quad \forall i \in V^{\prime}, c \in C_{E V}, k \in K_{E V}, i \neq j$

$$
\sum_{j \in V_{N+1}} x_{i j c k}^{C V}-\sum_{j \in V_{0}} x_{j i c k}^{C V}=0 \quad \forall i \in V, c \in C_{C V}, k \in K_{C V}, i \neq j
$$

Constraints 3.21 and 3.22 force each vehicle to be assigned to a maximum of one route.

$$
\begin{aligned}
& \sum_{j \in V^{\prime}} x_{0 j c k}^{E V} \leq 1 \quad \forall c \in C_{E V}, k \in K_{E V} \\
& \sum_{j \in V} x_{0 j c k}^{C V} \leq 1 \quad \forall c \in C_{C V}, k \in K_{C V}
\end{aligned}
$$

$F^{\prime}$ is a set of dummy nodes representing visits to each vertex in the set of charging stations, $F$. Constraint 3.23 ensures that each node in the set $F^{\prime}$ is visited at most once by each vehicle. This makes it possible for each charging station to be visited once, multiple times or not at all by the ECVs on the road.

$$
\sum_{j \in V_{n+1}} x_{i j c k}^{E V} \leq 1 \quad \forall c \in C_{E V}, k \in K_{E V}, \forall i \in F^{\prime}, i \neq j
$$

The travel times on arcs are linked through constraints 3.24 and 3.25, which ensure the connectivity of travel times on the traveled arcs by each vehicle. For ECVs, the time spent for charging at each charging station visit node is accounted for by treating the charging time as a service time for that node. 


$$
\begin{gathered}
a t_{j c k}^{E V} \geq a t_{i c k}^{E V}+\left(t_{i j}+S_{i}\right) \cdot x_{i j c k}^{E V}-T \cdot\left(1-x_{i j c k}^{E V}\right) \\
\in V^{\prime}{ }_{n+1}, \quad i \neq j \\
a t_{j c k}^{C V} \geq a t_{i c k}^{C V}
\end{gathered}
$$

Constraints 3.26 and 3.27 guarantee that the arrival time of vehicles at each node is within the planning period $\left[T_{0} \mathrm{~T}\right]$.

$$
\begin{aligned}
& T_{0} \leq a t_{j c k}^{E V} \leq T \quad \forall c \in C_{E V}, k \in K_{E V}, \forall j \in V^{\prime}{ }_{n+1} \\
& T_{0} \leq a t_{j c k}^{C V} \leq T \quad \forall c \in C_{C V}, k \in K_{C V}, \forall j \in V_{n+1}
\end{aligned}
$$

If the arrival of a vehicle at a demand node is not within the desired delivery time window, constraints 3.28 to 3.31 calculate the early or delayed service time at the demand node.

$$
\begin{array}{ll}
w_{j c k}^{E V} \geq e_{j}-a t_{j c k}^{E V} & \forall c \in C_{E V}, k \in K_{E V}, \forall j \in V \\
d_{j c k}^{E V} \geq a t_{j c k}^{E V}-l_{j} & \forall c \in C_{E V}, k \in K_{E V}, \forall j \in V \\
w_{j c k}^{C V} \geq e_{j}-a t_{j c k}^{C V} & \forall c \in C_{C V}, k \in K_{C V}, \forall j \in V \\
d_{j c k}^{C V} \geq a t_{j c k}^{C V}-l_{j} & \forall c \in C_{C V}, k \in K_{C V}, \forall j \in V
\end{array}
$$

Constraints 3.32 and 3.33 force the load of a vehicle over arc $(i, j)$ to be zero if the arc is not traversed by that vehicle which help linearize the objective function as explained is the previous section. The total load a vehicle carries is limited by its capacity through constraints 3.34 and 3.35 . 


$$
\begin{aligned}
& q_{j} \cdot x_{i j c k}^{E V} \leq U_{i j c k}^{E V} \leq\left(Q_{c}^{E V}-q_{i}\right) \cdot x_{i j c k}^{E V} \quad \forall c \in C_{E V}, k \in K_{E V}, i \in V_{0}, j \in V_{n+1} \\
& q_{j} \cdot x_{i j c k}^{C V} \leq U_{i j c k}^{C V} \leq\left(Q_{c}^{C V}-q_{i}\right) \cdot x_{i j c k}^{C V} \quad \forall c \in C_{C V}, k \in K_{C V}, i \in V_{0}, j \in V_{n+1} \\
& \sum_{i \in V} q_{i} \sum_{j \in V_{N+1}} x_{i j c k}^{E V} \leq Q_{c}^{E V} \quad \forall c \in C_{E V}, k \in K_{E V} \\
& \sum_{i \in V} q_{i} \sum_{j \in V_{N+1}} x_{i j c k}^{C V} \leq Q_{c}^{C V} \quad \forall c \in C_{C V}, k \in K_{C V}
\end{aligned}
$$

Balance of load flow at each node is defined through constraints 3.36 and 3.37. These constraints model the vehicle load flow as increasing by the amount of cargo demand of each visited demand node.

$$
\begin{array}{ll}
\sum_{j \in V^{\prime} 0} U_{j i c k}^{E V}-\sum_{j \in V^{\prime} N+1} U_{i j c k}^{E V}=q_{i} \sum_{j \in V^{\prime} N+1} x_{i j c k}^{E V} & \forall c \in C_{E V}, k \in K_{E V}, \forall i \in V \\
\sum_{j \in V_{0}} U_{j i c k}^{C V}-\sum_{j \in V_{N+1}} U_{i j c k}^{C V}=q_{i} \sum_{j \in V_{N+1}} x_{i j c k}^{C V} & \forall c \in C_{C V}, k \in K_{C V}, \forall i \in V
\end{array}
$$

In constraints 3.38 , the remaining battery capacity of all electric vehicles are set to their full battery capacity before starting their route. This means that all the vehicles leave the depot with fully charged batteries.

$$
R_{o c k=B C_{c}^{E V}} \quad \forall c \in C_{E V}, k \in K_{E V}
$$

Constraint 3.39 set the battery level of a vehicle arriving at a node succeeding a demand node in accordance with the energy consumption on the arc joining these two nodes. Constraints 3.40 define the same relation for the nodes succeeding a charging station. 


$$
\begin{gathered}
R_{j c k} \leq R_{i c k}-\left(\left({\propto^{\prime}}_{i j} \cdot t_{i j} \cdot w_{k}^{E V} \cdot x_{i j k}^{E V}+{\propto^{\prime}}_{i j} \cdot t_{i j} \cdot U_{i j k}^{E V}\right)+\beta_{i j}^{\prime} \cdot t_{i j}\right)+E C_{k}\left(1-x_{i j k}^{E V}\right) \\
\forall c \in C_{E V}, k \in K_{E V}, \forall i \in V, j \in V^{\prime}{ }_{n+1}, i \neq j \\
R_{j c k} \leq E C_{k}-\left(\left(\left(^{\prime}{ }_{i j} \cdot t_{i j} \cdot w_{k}^{E V} \cdot x_{i j k}^{E V}+\propto^{\prime}{ }_{i j} \cdot t_{i j} \cdot U_{i j k}^{E V}\right)+\beta_{i j}^{\prime} \cdot t_{i j}\right)\right. \\
\forall k \in K^{E V}, \forall i \in F^{\prime} \cup\{0\}, j \in V^{\prime}{ }_{n+1}, i \neq j
\end{gathered}
$$

Constraint 3.41 ensure that if an internal combustion engine vehicle visits any demand node in a Low Emission Zone, the decision variable, $y_{c k l}^{C V}$, is set to one for the vehicle and the corresponding zone. Finally, binary decision variables are defined in constraints 3.42 .

$$
\begin{aligned}
& \sum_{i \in l} \sum_{j \in V_{n+1}} x_{i j c k}^{C V} \leq M y_{c k l}^{C V} \quad \forall c \in C_{C V}, k \in K_{C V}, l \in L E Z_{l} \\
& x_{i j c k}^{E V}, x_{i j c k}^{C V}, y_{c k l}^{C V} \in\{0,1\}
\end{aligned}
$$

\section{$\underline{3.4 \text { Numerical Study }}$}

In this section a small size problem is presented and a number of scenarios will be examined to evaluate the features of the proposed mixed integer linear programming model. Xpress 7.9 software is used to solve this problem to optimality.

The network of small size problem is illustrated in Figure 3.3. The network consists of 10 demand points (shown as blue filled circles), 1 charging station, and 1 Depot (shown as a blue star). The available vehicles are 2 types of electric trucks with different loading and battery capacities (shown as green colored trucks) and 2 types of internal combustion engine trucks with different loading capacities. Travel speed, and average acceleration rate of each link in the network are randomly generated from uniform 
distributions between $[11,40]$ and $[0.1,1.2]$ respectively. The link travel times are estimated based on the randomly generated speed and distance for each link. The required customer service time at demand nodes are randomly generated from a uniform distribution between $\left[T_{0}, \frac{T}{5}\right]$, where $T_{0}$ is the starting time of the delivery operation and $T$ is the ending time of the operation. The customer demands are randomly generated from a uniform distribution between [500, 2500] lbs.

The formulated problem is solved using Xpress 7.9 for a number of case studies with different specifications on the small network presented in Figure 3.3. The detailed list of the cases is shown in Table 3.1. In all of the cases, it is assumed that all operated vehicles leave the depot at 8 am and all the ECVs are fully charged overnight. In the first case study the formulated problem is solved for a scenario where there is no limitation on emission and there is no LEZ in the network. The optimal solution to this problem shows the optimal fleet and routes required to serve the demand while there is no limitation on the employment of ICCVs. In case\#2 to case\#4, the effect of limiting emission for daily operation is studied for different prices of carbon. In these cases, it is assumed that the only limitation for using ICCVs is the carbon emission and there is no LEZ in the network. In case\#5 to case\#7, the limitation on the emission is replaced with LEZ. Different configurations of LEZs are studied and their effects on the fleet design and routes are explored. Having studied the effect of emission cap and LEZs separately on daily delivery operations, in case\#8 the impact of having both limitations is explored. At last, in case\#9, the problem formulation is solved for a new network configuration with two LEZs with different penalty costs. 


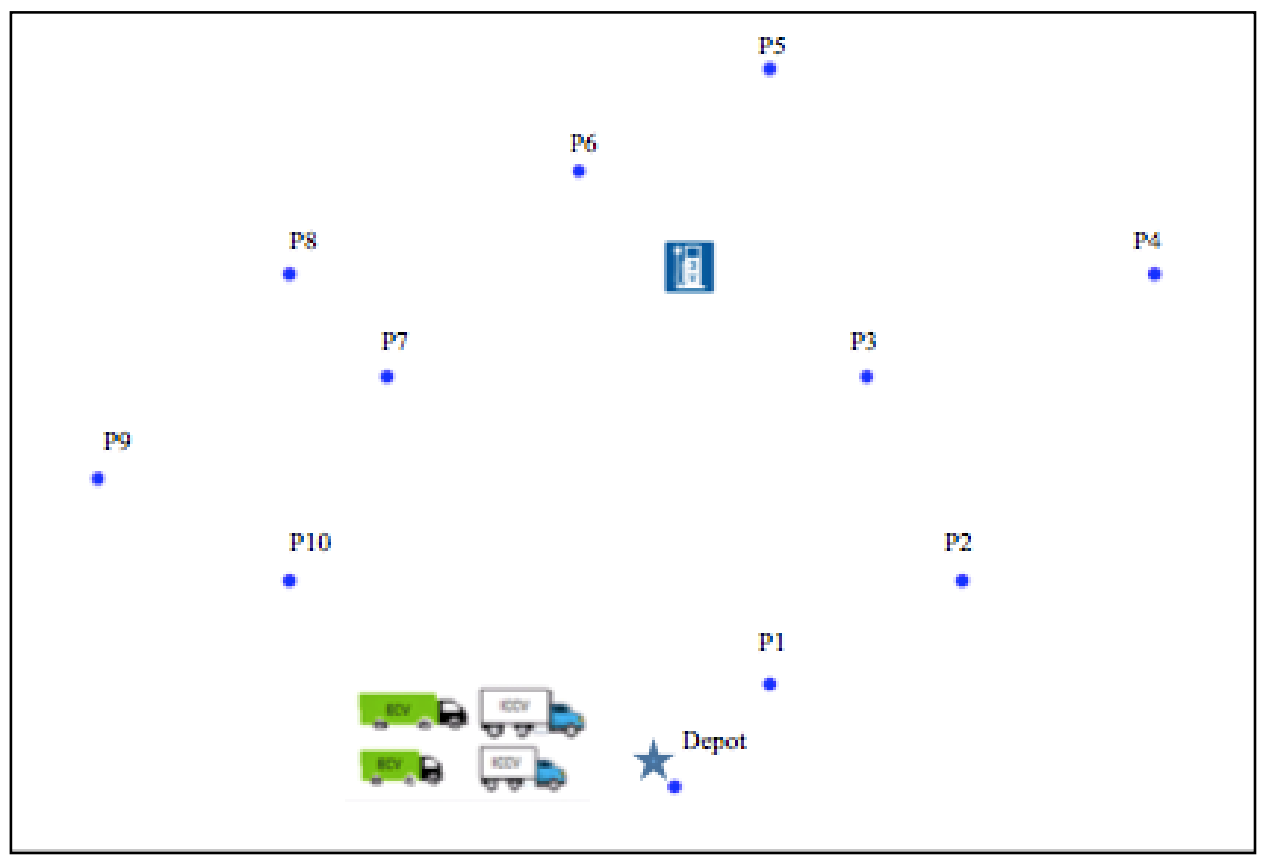

Figure 3.3. Small Size Problem Network Configurations

Table 3.1 Case Studies Specifications

\begin{tabular}{|c|c|c|c|c|c|}
\hline & $\begin{array}{c}\text { No. of } \\
\text { LEZs in } \\
\text { Network }\end{array}$ & Nodes in each LEZ & $\begin{array}{c}\text { LEZ } \\
\text { Penalty } \\
\text { Cost }\left(\frac{\$}{\text { day }}\right)\end{array}$ & $\begin{array}{c}\text { Emission } \\
\text { Cap }\left(\frac{\text { gram }}{\text { day }}\right)\end{array}$ & $\begin{array}{c}\text { Emission } \\
\text { Cost } \\
\left(\frac{\$}{\text { gram }}\right) \\
\end{array}$ \\
\hline Case \#1 & - & - & - & - & - \\
\hline Case \#2 & - & - & - & 50 grams & 0.50 \\
\hline Case \#3 & - & - & - & 50 grams & 0.25 \\
\hline Case \#4 & - & - & - & 50 grams & 0.10 \\
\hline Case \#5 & 1 & $\{\mathrm{P} 3, \mathrm{P} 4, \mathrm{P} 5\}$ & 100 & - & - \\
\hline Case \#6 & 1 & $\{\mathrm{P} 3, \mathrm{P} 4, \mathrm{P} 5, \mathrm{P} 6, \mathrm{P} 7, \mathrm{P} 8\}$ & 100 & - & - \\
\hline Case \#7 & 1 & All & 100 & - & - \\
\hline Case \#8 & 1 & $\{\mathrm{P} 2, \mathrm{P} 3, \mathrm{P} 4\}$ & 40 & 50 grams & 0.25 \\
\hline Case \#9 & 2 & $\begin{aligned} \text { LEZ1 } & =\{\mathrm{P} 3, \mathrm{P} 4, \mathrm{P} 5\} \\
\mathrm{LEZ2} & =\{\mathrm{P} 7, \mathrm{P} 8, \mathrm{P} 9\}\end{aligned}$ & $\begin{array}{l}\mathrm{P}_{\text {LEZ1 }}=\$ 40 \\
\mathrm{P}_{\text {LEZ2 }}=\$ 10\end{array}$ & 50 grams & 0.25 \\
\hline
\end{tabular}




\subsubsection{Case \#1}

In the first case study, the problem is solved for a network with no low emission zones (LEZ) and no limitation on the carbon emission. The only limitation accounted for is the ECV's range limitation due to its limited battery capacity. Based on these assumptions, the given network and characteristics of the problem, Xpress 7.9 is used to find the solution of the problem in terms of the optimal fleet design and the optimal routes for each used vehicle. Figure 3.4 shows the optimal solution to the problem. As it can be seen in the figure, when there is no limitation on the employment of ICCVs, the optimal fleet is composed of two ICCVs, one with lower capacity and the other one with higher capacity. The total routing polluted emission by the delivery operation is calculated as 159.74 grams.

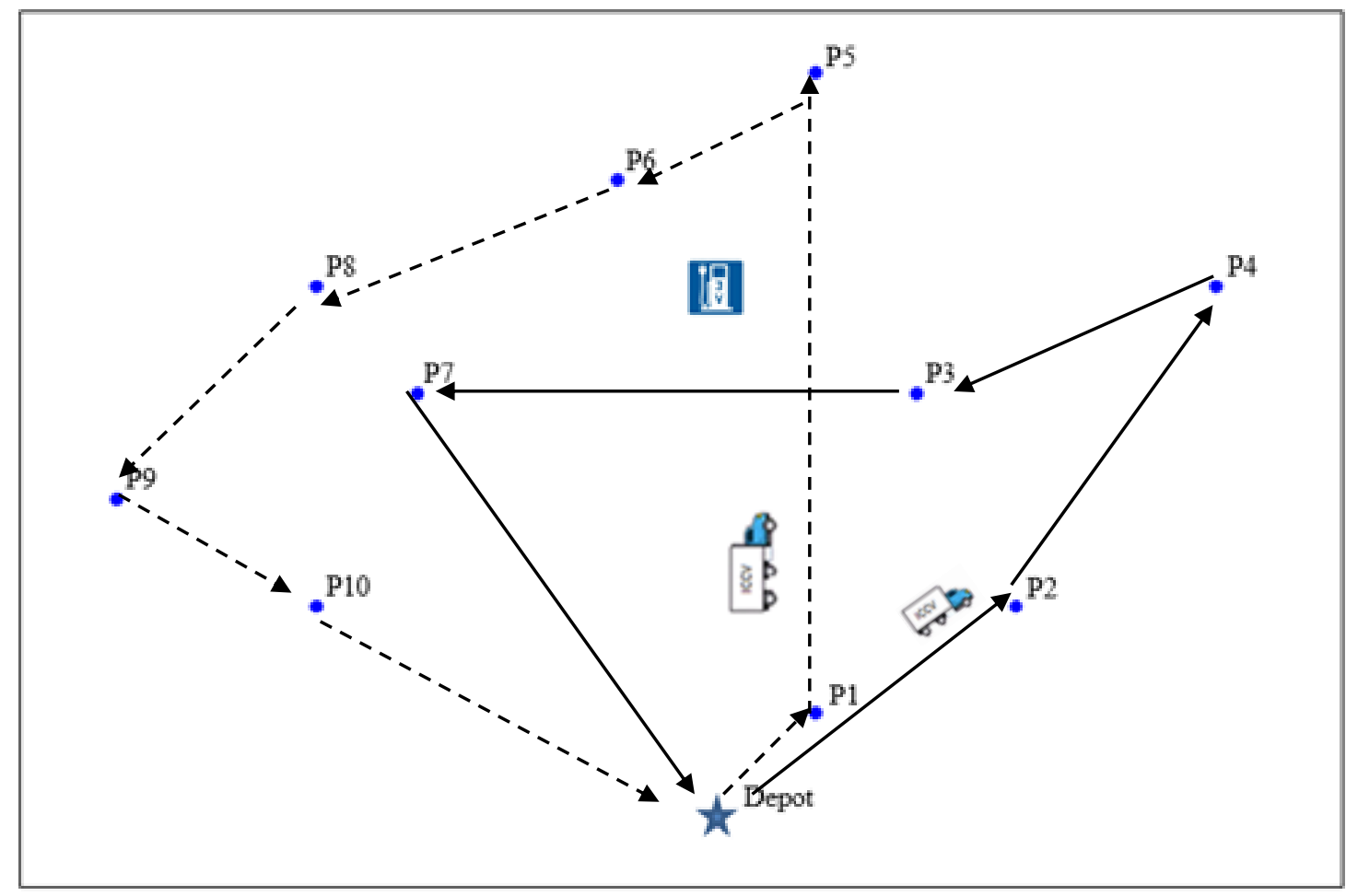

Figure 3.4. Case \#1 Optimal Solution 


\subsubsection{Case \#2}

In this case study, a limit has been imposed on the emission. It is assumed that there is a limit of 50 grams on emission of the company daily operations. It means that the permission to produce any amount of emission over this limit should be bought with the price of $\frac{\$ 0.50}{\text { gram }}$. On the other hand, if the total daily-emitted pollution is less than the limit, the extra carbon points can be sold with the same price to other companies. Figure 3.5 shows the optimal solution to the problem. As it can be seen in the figure, by adding a limit on emission, the optimal fleet is changed to two ECVs of different sizes to serve the demand. The ECVs routes consist of one visit to the charging station for recovering their full battery capacity. As a result of the change in fleet the total routing polluted emission by the delivery operation is reduced to zero.

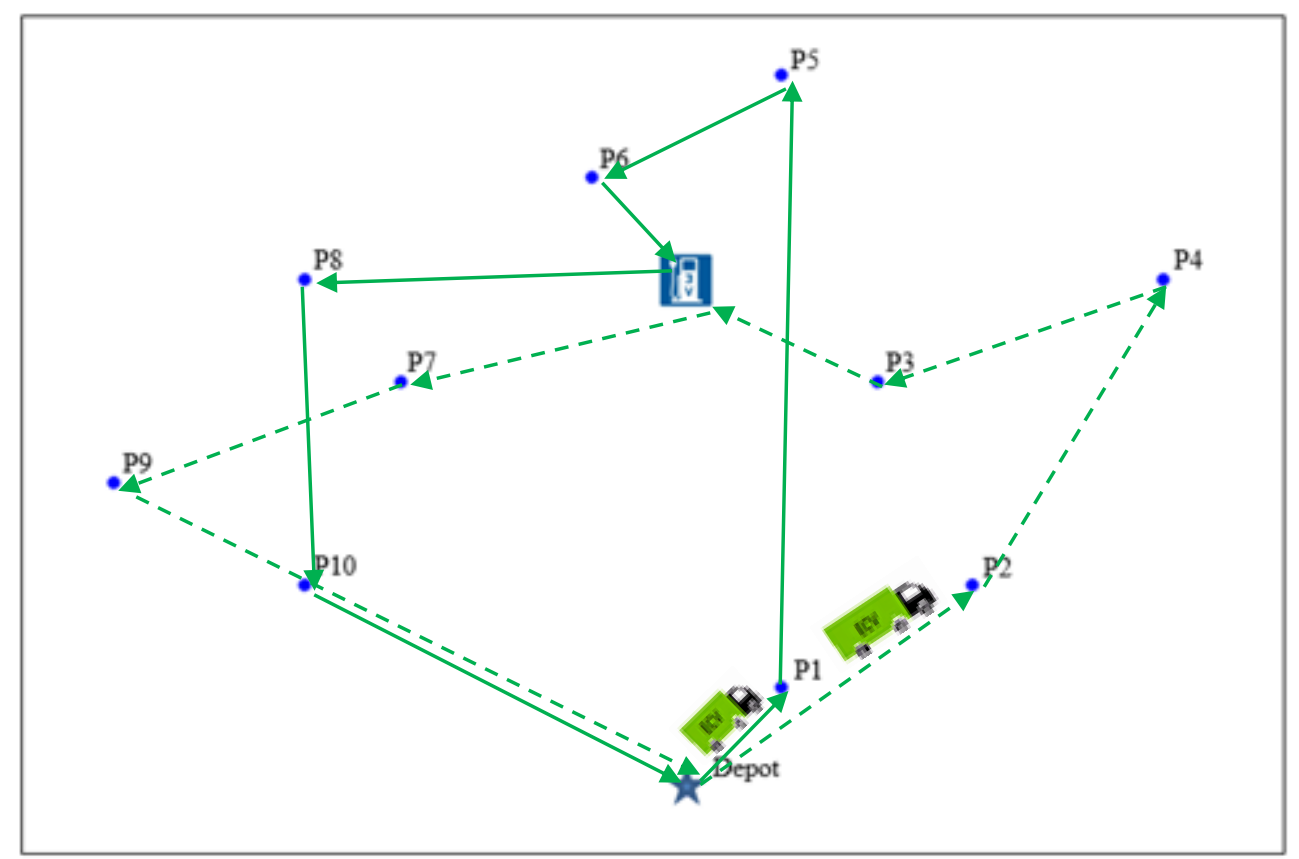

Figure 3.5 Case\#2 Optimal Solution 


\subsubsection{Case \#3}

In the third example, all the assumptions and characteristics of the previous example is held except for the price of carbon. In this example, it is assumed that the carbon price is decreased to $\frac{\$ 0.25}{\text { gram }}$. Figure 3.6 shows the optimal solution to this problem. As it can be seen in this figure, by reducing the price of the carbon by 50\%, the optimal fleet and routes are changed. In fact, the ECV with smaller capacity is replaced with an ICCV with the same loading capacity. The majority of demand is still served with ECV due to its lower operation cost and zero emissions, and, as a result of this change in fleet and routing, the delivery operation related emissions is increased from zero to 62.61 grams.

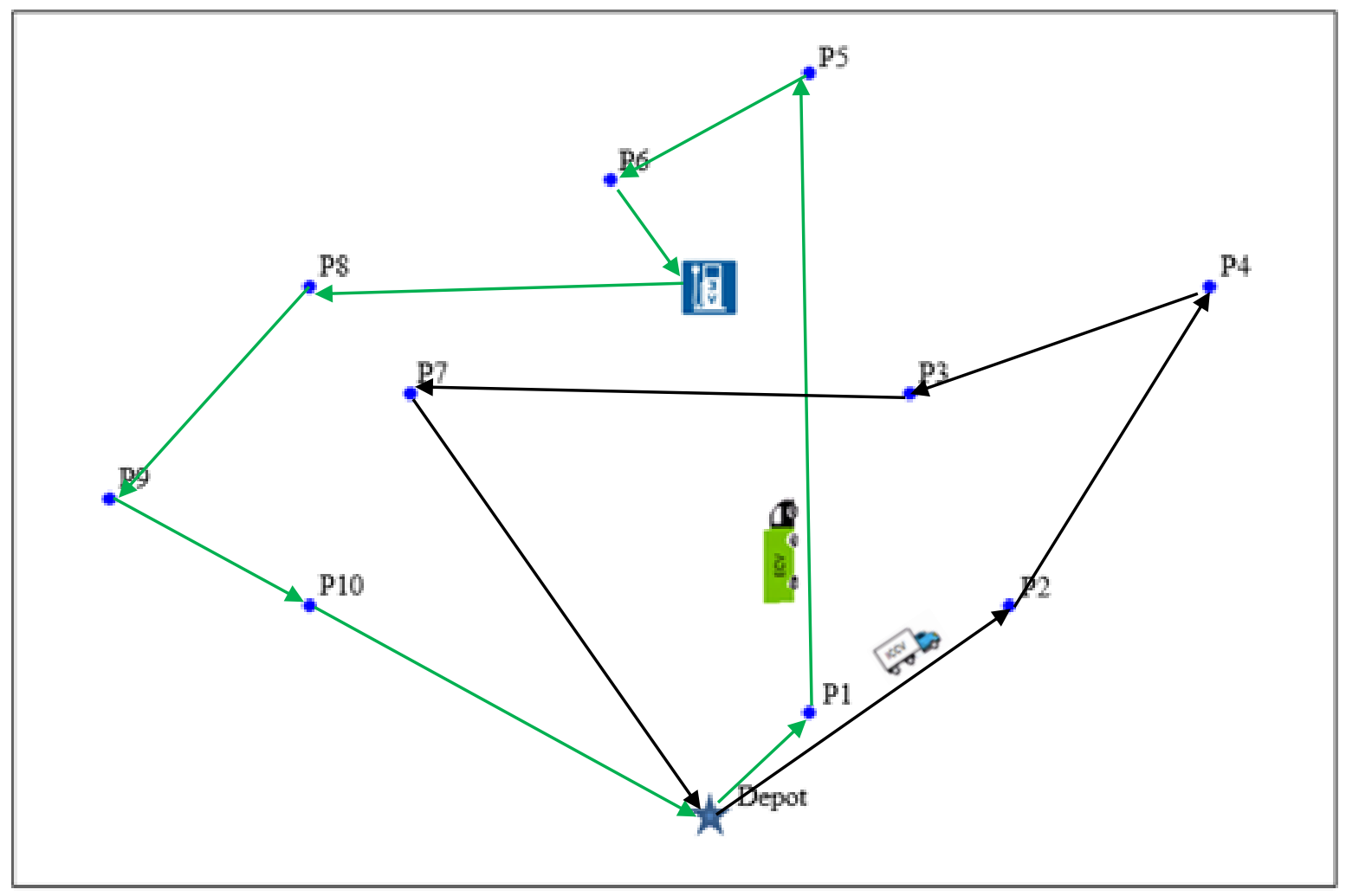

Figure 3.6. Case \#3 Optimal Solution 


\subsubsection{Case \#4}

In order to further investigate the effect of carbon price on fleet design and routing, in this

example, the price of carbon is further decreased to $\frac{\$ 0.10}{\text { gram }}$ but still there is no LEZ in the network. The optimal solution to this problem shows that, in the case that a limit exists on daily emission but the carbon price is low, there will be no change in the fleet design in comparison to the case that there is no limitation on emission. The fleet will be composed of two ICCVs with the same optimal routes found in case \#1, shown in Figure 3.4, and the total routing related emission will increase from 62.61 grams (when the price of carbon is $\frac{\$ 0.25}{\text { gram }}$ ) to 159.74 grams.

\subsubsection{Case \#5}

While previous cases were focused on the effect of carbon limits on fleet design and vehicle routing, in this example, the effect of having LEZ in the network is investigated. It is assumed that there is no carbon limit on daily operations but there exists a low emission zone in the network which encompasses $\% 30$ of demand nodes (nodes P3, P4, and P5). A one-time daily penalty of $\$ 100$ is considered for internal combustion engine trucks entering the LEZ in the network. Figure 3.7 shows the optimal solution to the problem. As it can be seen in the figure, the optimal fleet is composed of one ECV and one ICCV. Besides the demand nodes in the LEZ, the demand nodes of P1, P2, and P6 are served by the ECV too such that its maximum loading capacity is used as the operation cost of ECV is less that of the ICCV. The ECV's route consists of one visit to the charging 
station for recovering its full battery capacity. The total routing polluted emission by the delivery operation is calculated as 54.81 grams.

\section{$\underline{3.4 .6 \text { Case \#6 }}$}

In this example, the LEZ is expanded such that it contains $60 \%$ of demand nodes (P3, P4, P5, P6, P7, and P8) with the same penalty of $\$ 100$ for ICCVs to travel within the zone. The optimal solution to this example shows that the higher number of nodes in the LEZ result in the more number of employed ECVs. Since, the sum of the demands in the LEZ is more than the capacity limits of each type of available ECVs, both of them are used to avoid paying the high LEZ penalty cost and the total emission is reduced to zero. The optimal routes assigned to each ECV are the same as the ones calculated in case \#2 (Figure 3.5) where there was no LEZ in the network but a limit was imposed on the emission with carbon price of $\frac{\$ 0.50}{g r a m}$. 


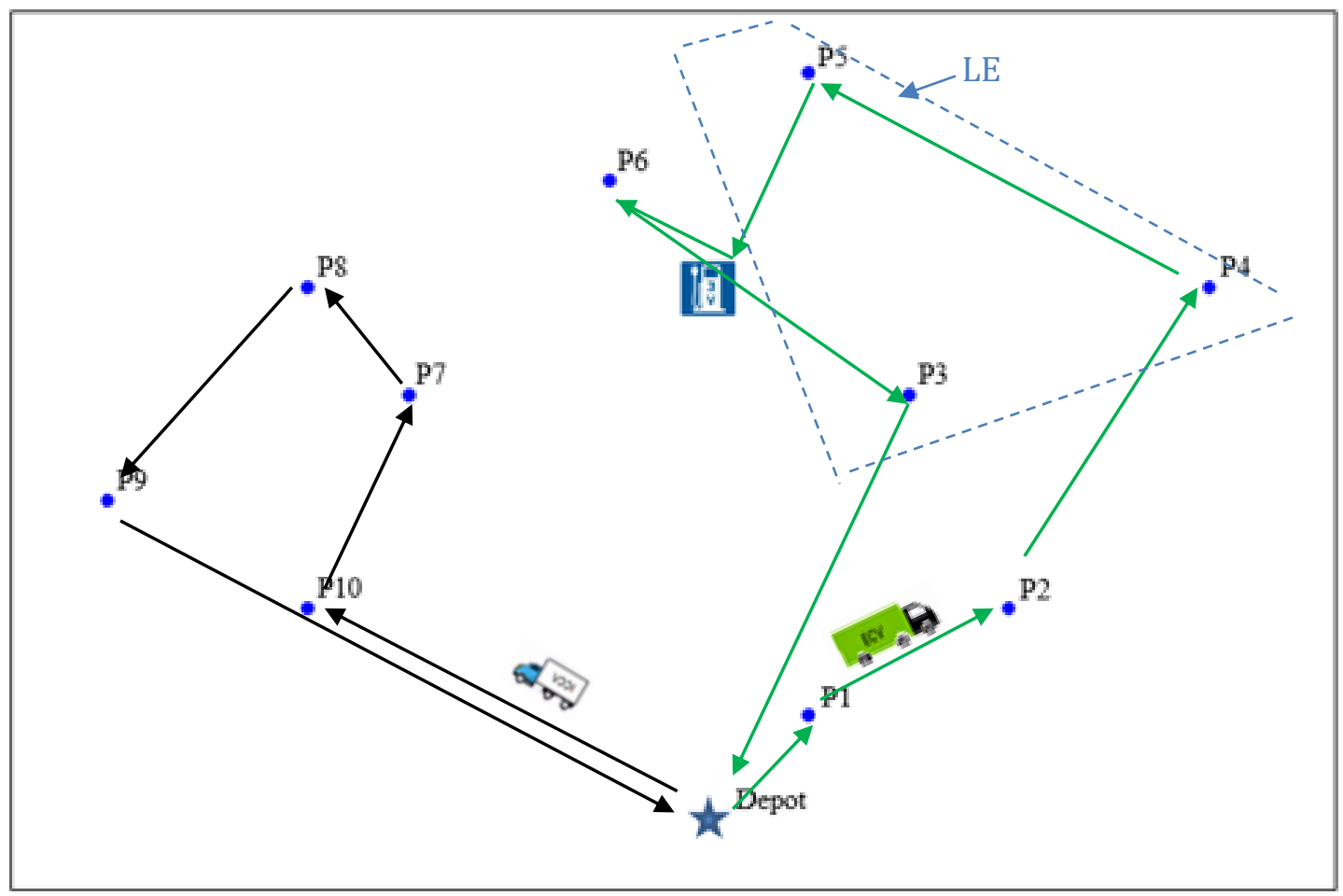

Figure 3.7. Case \# 5 Optimal Solution

\section{$\underline{\text { 3.4.7 Case \#7 }}$}

In the cases 5 and 6 , it was found that expansion of LEZ result in an increase in the number of employed ECVs. In this example, it is assumed that all demand nodes reside in LEZ. As it is expected, the optimal solution shows that having all demand points in the LEZ result in a pure fleet of ECV with optimal routes similar to case\#2 and case\#6 (Figure 3.5) where we have either emission cap with high carbon price or demands in LEZ exceeding the loading capacity of each available ECV. 


\section{$\underline{3.4 .8 \text { Case \#8 }}$}

Having studied the effects of emission cap and low emission zone separately on the fleet design and routing, in this example the impact of having a combination of both limitations is studied. It is assumed that there is a daily pollution limit of 50 grams on the delivery operation of the company. The price of carbon is set to $\frac{\$ 0.25}{\mathrm{gram}}$. Also, it is assumed that there is an LEZ in the network containing demand nodes P3, P4, and P5, with the penalty of $\$ 40$ for ICCVs. The optimal solution to this example is found to be same as case\#5 (Figure 3.7), where there is no emission cap on the operations but the penalty of driving within LEZ for ICCVs is $\$ 100$. The optimal fleet is composed of one ECV and one ICCV. The demand nodes in the LEZ are served by the ECV and the ECV's route consists of one visit to the charging station for recovering its full battery capacity. The vehicle with higher capacity is chosen to be of the electric type so that the majority of customers are served by ECV due to its lower operation cost and the savings that can be made by spending less on carbon permits. The total routing emission in this case is estimated to be 54.81 grams.

\section{$\underline{3.4 .9 \text { Case \#9 }}$}

In this case study, it is assumed that there are two LEZs in the network with different penalty costs for ICCVs. One of them which is closer to the charging station has higher penalty of $\$ 40$ and the other one has a lower penalty of $\$ 20$. There is also an emission cap of 50 grams imposed on the emitted pollutants with the carbon price of $\frac{\$ 0.25}{\text { gram }}$. The optimal solution to the problem is shown in Figure 3.8. The demand points in the LEZ with higher fine and the demand points in their vicinity are served with a big ECV such that its capacity 
is used to its maximum limit. The demand points in the other LEZ, with lower fine, are served with a small ECV as the penalty cost is less than the price difference of ECV and ICCV plus the cost of the emitted carbon to serve the nodes in the area.

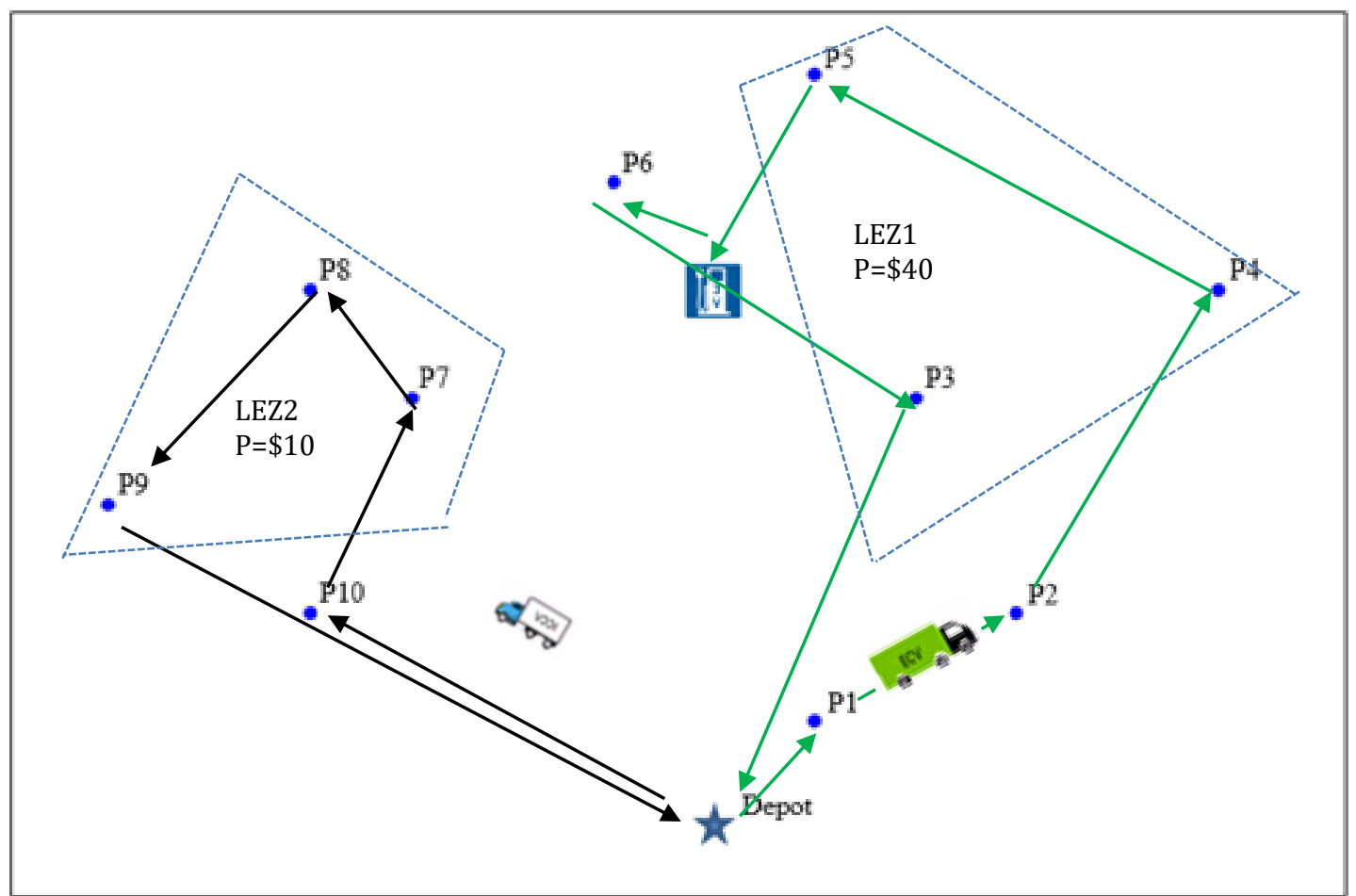

Figure 3.8. Case \#9 Optimal Solution

\section{$\underline{3.5 \text { Summary }}$}

In this chapter the GVRP with constant travel times was formulated. Problem characteristics were explained. The assumptions used in this research were introduced and the developed mathematical model was presented with a brief explanation of the objective function and constraints. A very small size problem was presented and solved for nine different scenarios to show the capabilities of the optimization model. These scenarios were solved using Xpress 7.9 and optimal solutions were shown and discussed. The 
results of the analysis on these three different problem instances showed the capability of the model to account for the limitations of the ECVs and ICCVs in finding the optimal fleet design and routes. It was shown that based on the different tradeoffs between the penalty costs of ICCV employment, and purchase cost and range limitation of the ECV different fleet composition and routings are found to be optimal. 


\section{Chapter 4}

\section{MODEL FORMULATION - TIME-DEPENDENT GVRP}

In this Chapter a mathematical model is formulated for the Time-Dependent GVRP explained problem in section 1.2 where travel time on arcs is dependent on the time of day. The problem is formulated as a mixed integer linear programming problem on a network. First, the problem properties are described completely. Then, the assumptions and limitations of the model are given. In the third section, the mathematical formulation of the problem is explained which includes detailed explanations of the notations and variables used in the model, the objective function, and the constraints. At last, the developed model is solved on a small size network problem. The problem is solved for both cases of static and Time-Dependent travel times and the results are compared. The chapter is summarized in the last section.

\subsection{Characteristics of the Problem}

\subsubsection{Network}

The Time-Dependent GVRP is formulated on a complete directed graph $G=\left(V_{0, N+1}, A\right)$.

Vertices $O$ and $N+1$ denote instances of depot. $V^{\prime}$ is the union of the set of demand nodes $(V=\{1,2, \ldots, N\})$ and the set of charging station visit nodes $\left(F^{\prime}\right)$, which represent the set of visits to vertices in the set of charging stations, $F$. All vehicle routes start from node 0 
and end at node $N+1$. The set of arcs is given by $A=\left\{(i, j) \mid(i, j) \in V_{0, N+1}^{\prime}, i \neq j\right\}$. Each arc is described by its travel time as a function of departure time, average travel speed $\left(V_{i j}^{t}\right)$, and average acceleration rate $\left(a_{i j}^{t}\right)$ for different time periods of a day. The travel time function, average travel speed and average acceleration rate on an arc are not only different for different time periods, but also they vary on different directions of an arc to represent the real world situation.

\subsubsection{Time-Dependent Travel Times}

In literature different approaches have been used to take into account the time dependency of travel times while finding solutions to variants of VRP. In 1992 Malandraki and Daskin used step functions to represent the variation of travel time along different time periods of a day. An example of a step function for travel time is given in Figure 4.1. This figure shows that while using a step function helps to account for variations in travel time, it might fail to take into account the First In First Out (FIFO) concept. Based on the FIFO concept if two vehicles leave from the same location for the same destination traveling on the same path, the one that leaves first will always arrive first, no matter how speed changes along the arcs during the travel. Therefore, in Figure 4.1, the vehicles departing at 11:01 are expected to arrive after the vehicles departing at 10:55. However, the use of step function with the sudden drop in travel time violates the FIFO concept.

The FIFO principle is very important as it prevents inconsistencies caused by vehicles waiting at some locations for the time when speeds are higher and then arrive at 
destination before than the vehicles which had left before the time when the speeds were lower. Moreover, due to the large effect of travel speed on vehicle energy consumption, a model that satisfies the "non-passing" property not only calculates total transportation time more accurately, but also estimates the vehicle energy requirement with more accuracy which is of high priority in this dissertation due to the sensitivity of the ECV's driving range to the battery consumption rate.

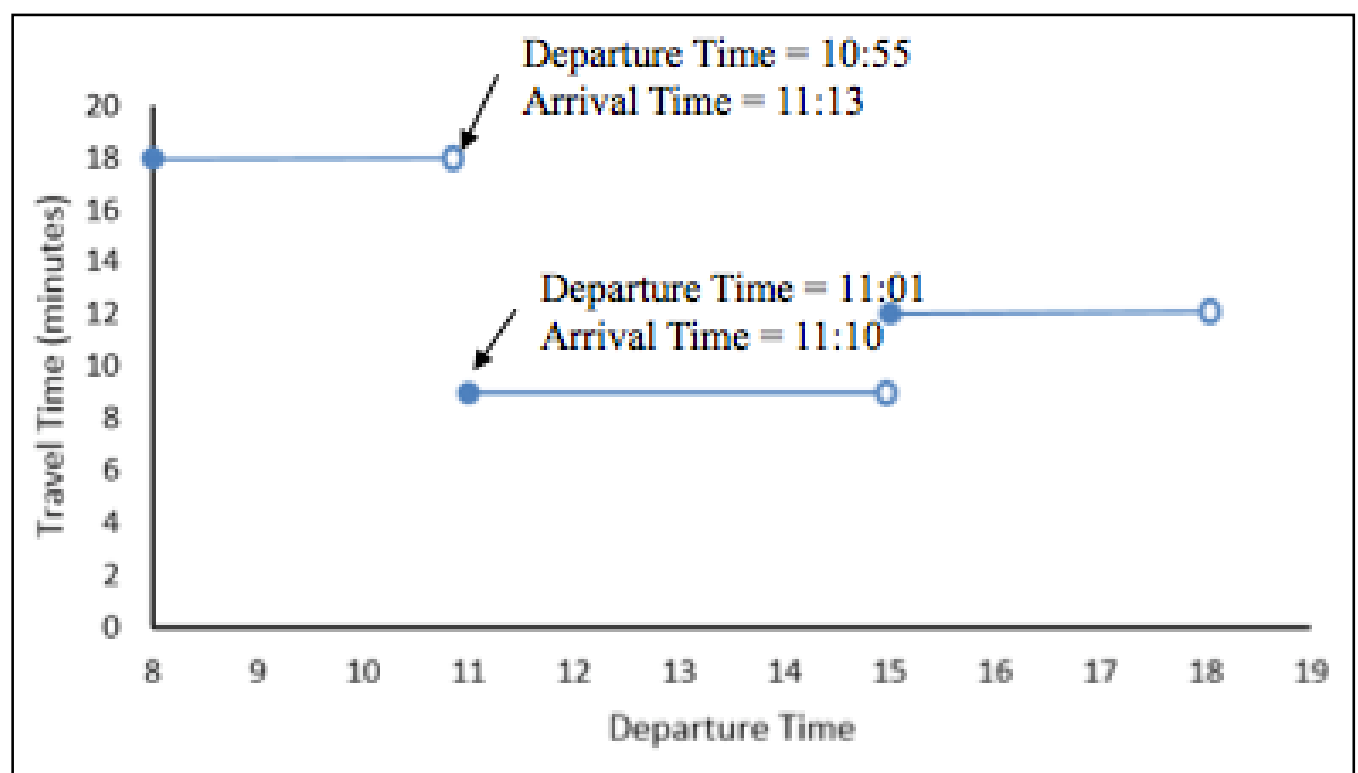

Figure 4. 1 Travel Time Step Function

In this study, in order to preserve the FIFO concept, it is assumed that travel time along each arc of the network is represented by a continuous function of time of a day as shown in Figure 4.2. Therefore, any type of variations in travel time can be accepted and taken into account by the model developed in this study. As it can be seen in this figure, 
travel time is a nonlinear function of time of a day. Piecewise linear functions can be used to estimate travel time as a linear function of time of a day during different time windows.


Figure 4. 2 Travel Time as a Continuous Function of Time of a Day

In this study it is assumed that all trucks leave the depot at $8 \mathrm{AM}$ and return back to depot at 6 PM. Therefore, travel time function was estimated for three time periods of morning rush hour, midday off-peak, and afternoon rush hour as shown in Figure 4.3. Each time window $t$, is represented by its range as $\left[L T^{t}, U T^{t}\right]$ and its travel time function in the form of: 
$t t^{t}=$ intercept $^{t}+$ slope $^{t} \times\left(\right.$ DepartureTime $\left.-L T^{t-1}\right) \quad t \in\{1,2,3\}$

where, intercept $t^{t}$ and slope $e^{t}$ are the intercept and slope of the fitted line at time window $t, L T^{t}$ is the lower bound of time window $t$. It should be noted that in this case, the FIFO concept is guaranteed only if the absolute value of the slope of this linear function is always less than 1 (Balseiro et al., 2011).

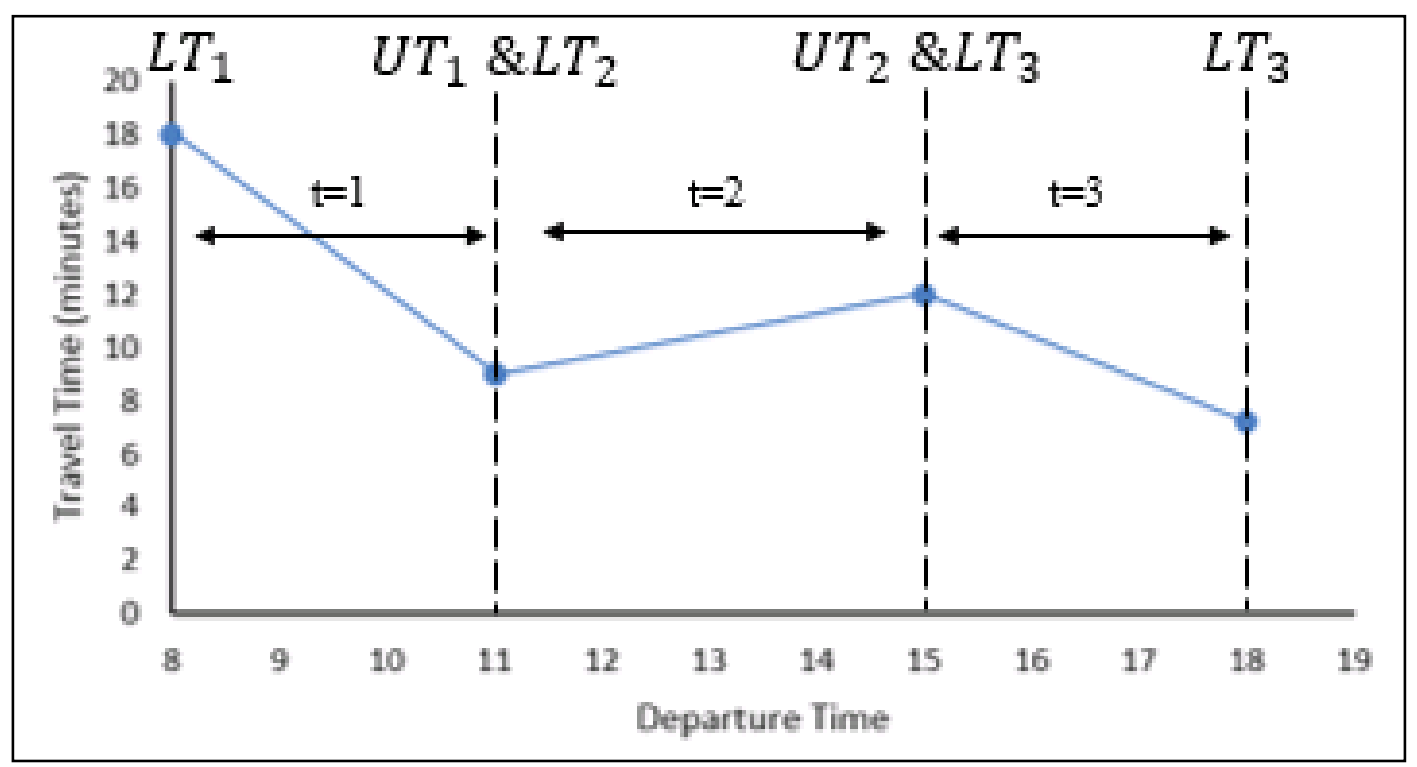

Figure 4. 3 Piecewise Linear Functions of Travel Time over the Planning Period

\subsubsection{Demand Nodes and charging nodes}

A nonnegative demand, $q_{i}$, and a nonnegative service time, $s_{i}$ is associated with each demand node in set $V$. There is also a service time window $\left[e_{i}, l_{i}\right]$ for each demand node within which the service to a customer has to start. Each charging station has a nonnegative service time and there is no specific time window for charging stations operating hours. 


\subsubsection{Commercial Vehicle Types}

A mixed fleet of heterogeneous electric commercial vehicles, ECVs, with different battery and loading capacity, and heterogeneous Internal Combustion Engine Vehicles, ICCVs, with different loading capacities is considered to be available in this study. The number of vehicles is not predefined and it is one of the objectives of the problem to find the optimal number of vehicles of each type to be used to serve the demand.

\subsubsection{Energy Consumption of Electric Commercial Vehicles}

The energy requirement of an electric commercial truck is estimated using the equations explained in section 3.1.4. In these equations, average speed and average acceleration rate during different time windows are used to estimate the required mechanical power. Also, travel times are estimated using the departure time and travel time function as explained in section 4.1.2.

\subsubsection{Energy Consumption of Internal Combustion Commercial Vehicles}

The energy requirement of a conventional commercial truck is estimated using the equations explained in section 3.1.5. Again, the average speed and the average acceleration rate during different time windows are used to estimate the required mechanical power. Travel times are estimated using the travel time functions. 


\subsubsection{Emission Model}

The emission produced by a conventional commercial truck is estimated using the equations explained in section 3.1.6.

\subsection{Assumptions}

\subsubsection{Customer Service Time Window}

The service time windows associated with demand points in the network are assumed to be soft time windows. There is a time penalty associated with early or delayed services meaning that if the demands are not served on time, a delay or waiting penalty is imposed.

\subsubsection{Charging Station Type}

As explained before, the charging stations are assumed to be of battery swapping type meaning that once an ECV reaches a charging station its battery is swapped with a fully charged battery. Therefore, the charging time is assumed to be the same for all types of ECVs at all charging station locations.

\subsubsection{Government Incentives and Regulations}

It is assumed that a) there is a financial incentive by government subsidizing the purchase of ECVs, b) there is a number of Low Emission Zones (LEZ) in the network operating 24 hours a day and 365 days of a year such that combustion engine commercial vehicles are required to pay a daily charge to drive within these zones, c) there is an emission cap imposed by the government on the amount of pollution that a company can produce in a year. 


\subsection{Mathematical Formulation}

The Time-Dependent GVRP of interest is formulated as a mixed integer linear programming problem based on the above assumptions. In the following subsections, the notations, coefficients, and variables used in the model are introduced and the objective function and constraints are explained.

\subsubsection{Notation and Variables}

\section{$\underline{\text { Data Sets }}$}

$V \quad$ Set of demand nodes

$F^{\prime} \quad$ Set of Charging Station visit nodes, dummy vertices of the set of charging stations $F$

$V^{\prime} \quad V \cup F^{\prime}$

$V_{0}, V_{N+1}$ Instances of depot

$V_{0, N+1} \quad V \cup V_{0} \cup V_{N+1}$

$V_{0, N+1}^{\prime} \quad V^{\prime} \cup V_{0} \cup V_{N+1}$

$L E Z_{l} \quad$ Set of demand nodes in the low emission zone $l \in\{1,2, \ldots, L\}$

$C_{C V} \quad$ Set of ICCV types

$C_{E V} \quad$ Set of ECV types

\section{$\underline{\text { Constants }}$}

$q_{i} \quad$ Nonnegative demand of node $i \in V$

$e_{i} \quad$ Earliest service time of node $i \in V$ 
$l_{i} \quad$ Latest service time of node $i \in V$

$S_{i} \quad$ Service time of node $i \in V^{\prime}$

$F C_{c}^{E V} \quad$ Fixed cost of EV type $c \in C_{E V}\left(\frac{\$}{d a y}\right)$

$F C_{c}^{C V} \quad$ Fixed cost of ICCV type $c \in C_{C V}\left(\frac{\$}{d a y}\right)$

$Q_{c}^{E V} \quad$ Loading capacity of EV type $c \in C_{E V}$

$Q_{c}^{C V} \quad$ Loading capacity of ICCV type $c \in C_{C V}$

$W_{c}^{E V} \quad$ Weight of empty EV of type $c \in C_{E V}$

$W_{c}^{C V} \quad$ Weight of empty ICCV of type $c \in C_{C V}$

$B C_{c}^{E V} \quad$ Battery Capacity of EV type $c \in C_{E V}$

$C^{E} \quad$ Cost of Electricity $\left(\frac{\$}{k w}\right)$

$C^{F} \quad$ Cost of Fuel $\left(\frac{\$}{\text { liter }}\right)$

$C^{L} \quad$ Cost of Labor

$v_{i j}^{t} \quad$ Average travel speed on $\operatorname{arc}(i, j) \mid(i, j) \in V_{0, N+1}^{\prime}, i \neq j$, at time interval $t \in T$

$a_{i j}^{t} \quad$ Average acceleration rate on $\operatorname{arc}(i, j) \mid(i, j) \in V_{0, N+1}^{\prime}, i \neq j$, at time interval $t \epsilon$

WP Waiting time penalty

DP Delay time penalty

$P \quad$ LEZ daily penalty

$e^{C A P} \quad$ Emission Cap

$P_{\text {Carb }} \quad$ Price of carbon $\left(\frac{\$}{\text { gram }}\right)$ 
$P B \quad$ Battery Cycle Cost

ghg Greenhouse Gas emissions per liter of fuel $\left(\frac{\text { gram }}{\text { liter }}\right)$

$L T_{t} \quad$ Lower boundary of time period $t \in T$

$U T_{t} \quad$ Upper boundary of time period $t \in T$

$L U_{m} \quad$ Lower boundary of load interval $m \in M$

$U U_{m} \quad$ Upper boundary of load interval $m \in M$

$\bar{U}_{m} \quad$ Average of load in load interval $m \in M$

$T_{0} T_{E n d}$ Start and End time of delivery operations

\section{Decision Variables}

$=1$, if ECV $k$ of type $c$ travels from node $i$ to node $j$ in time interval $t$ carrying

$x_{i j c k t m}^{E V} \quad$ load in interval $m$

$=0$, otherwise

$=1$, if ICCV $k$ of type $c$ travels from node $i$ to node $j$ in time interval $t$ carrying

$x_{i j c k t m}^{C V} \quad$ load in interval $m$

$=0$, otherwise

$=1$, if combustion engine vehicle $k$ of type $c$ enters low emission zone $l$

$y_{c k l}^{C V}$

$=0$, otherwise

$a t_{i c k}^{E V} \quad$ Arrival time of electric vehicle $k$ of type $c$ at node $i$

$a t_{i c k}^{C V} \quad$ Arrival time of combustion engine vehicle $k$ of type $c$ at node $i$

$d t_{i c k}^{E V} \quad$ Departure time of electric vehicle $k$ of type $c$ from node $i$ 
$D T_{i j c k t m}^{E V}$ Departure time of ECV $k$ of type $c$ from node $i$ to node $j$ in time interval $t$ carrying load in interval $m$

$D T_{i j c k t m}^{C V}$ Departure time of ICCV $k$ of type $c$ from node $i$ to node $j$ in time interval $t$ carrying load in interval $m$

$U_{i j c k}^{E V} \quad$ Load carried by electric vehicle $k$ of type $c$ from node $i$ to node $j$

$U_{i j c k}^{C V} \quad$ Load carried by combustion engine vehicle $k$ of type $c$ from node $i$ to node $j$

$R_{i c k} \quad$ Remaining battery of electric vehicle $k$ of type $c$ upon arrival at node $i$

$w_{i c k}^{E V} \quad$ Waiting time of electric vehicle $k$ of type $c$ at node $i$

$w_{i c k}^{C V} \quad$ Waiting time of combustion engine vehicle $k$ of type $c$ at node $i$

$d_{i c k}^{E V} \quad$ Delayed time of electric vehicle $k$ of type $c$ at node $i$

$d_{i c k}^{C V} \quad$ Delayed time of combustion engine vehicle $k$ of type $c$ at node $i$

\subsubsection{Objective Function}

The objective is to minimize the total vehicle purchase cost, fuel and electric energy consumption cost, labor cost and the total LEZ, service time, and carbon penalty cost.

Minimize:

$\sum_{c \in C^{E V}} \sum_{k \in S_{c}^{E V}} \sum_{j \in V^{\prime}} \sum_{t \in T} \sum_{m \in M} F_{c}^{E V} \cdot x_{0 j c k t m}^{E V}+$ $\sum_{c \in C} \sum_{k \in S_{C}^{C V}} \sum_{j \in V} \sum_{t \in T} \sum_{m \in M} F_{C}^{C V} \cdot x_{0 j c k t m}^{C V}+$ 


$$
\begin{aligned}
& C^{F} \times \sum_{c \in C} \sum_{k \in S_{c}^{C V}} \sum_{j \in V} \sum_{t \in T}\left(\left(\propto_{i j} \times w_{c}^{C V}+\beta_{i j}\right) \times\left(\text { intercept }_{i j}^{t} \times \sum_{m} x_{i j c k t m}^{C V}\right.\right. \\
& \left.+\operatorname{slope}_{i j}^{t} \times \sum_{m}\left(D T_{i j c k t m}^{C V}-L T_{t} \times x_{i j c k t m}^{C V}\right)\right) \\
& +\propto_{i j} \times\left(\text { intercept }_{i j}^{t} \times \sum_{m} M_{a v g} \times x_{i j c k t m}^{C V}\right. \\
& \left.\left.+\operatorname{slope}_{i j}^{t} \times \sum_{m} \bar{U}_{m} \times\left(D T_{i j c k t m}^{C V}-L T_{t} \times x_{i j c k t m}^{C V}\right)\right)\right)+ \\
& C^{E} \times \sum_{c \in C^{E V}} \sum_{k \in S_{c}^{E V}} \sum_{j \in V^{\prime}} \sum_{t \in T}\left(\left(\propto^{\prime}{ }_{i j} \times w_{c}^{E V}\right.\right. \\
& \left.+\beta_{i j}^{\prime}\right) \times\left(\text { intercept }_{i j}^{t} \times \sum_{m} x_{i j c k t m}^{E V}+\operatorname{slope}_{i j}^{t} \times \sum_{m}\left(D T_{i j c k t m}^{E V}\right.\right. \\
& \left.\left.-L T_{t} \times x_{i j c k t m}^{E V}\right)\right)+\alpha^{\prime}{ }_{i j} \times\left(\text { intercept }_{i j}^{t} \times \sum_{m} M_{a v g} \times x_{i j c k t m}^{E V}\right. \\
& \left.\left.+\operatorname{slope}_{i j}^{t} \times \sum_{m} \bar{U}_{m} \times\left(D T_{i j c k t m}^{E V}-L T_{t} \times x_{i j c k t m}^{E V}\right)\right)\right)+
\end{aligned}
$$$$
P \sum_{c \in C^{C V}} \sum_{k \in S_{C}^{C V}} \sum_{l \in L} y_{k l}^{C V}+
$$$$
W P\left(\sum_{i \in V} \sum_{c \in C^{E V}} \sum_{k \in S_{c}^{E V}} w_{i c k}^{E V}+\sum_{i \in V} \sum_{c \in C^{C V}} \sum_{k \in S_{c}^{C V}} w_{i c k}^{C V}\right)+
$$$$
D P\left(\sum_{i \in V} \sum_{c \in C^{E V}} \sum_{k \in S_{c}^{E V}} d_{i c k}^{E V}+\sum_{i \in V} \sum_{c \in C^{C V}} \sum_{k \in S_{c}^{C V}} d_{i c k}^{C V}\right)-
$$ 


$$
\begin{aligned}
& P_{\text {carb }}\left(e^{C A P}-g h g\left[\sum _ { c \in C } \sum _ { k \in S _ { C } ^ { C V } } \sum _ { j \in V } \sum _ { t \in T } \left(\left(\propto_{i j} \times w_{c}^{C V}\right.\right.\right.\right. \\
& \left.+\beta_{i j}\right) \times\left(\text { intercept }_{i j}^{t} \times \sum_{m} x_{i j c k t m}^{C V}+\operatorname{slope}_{i j}^{t} \times \sum_{m}\left(D T_{i j c k t m}^{C V}\right.\right. \\
& \left.\left.-L T_{t} \times x_{i j c k t m}^{C V}\right)\right)+\propto_{i j} \times\left(\text { intercept }_{i j}^{t} \times \sum_{m} M_{a v g} \times x_{i j c k t m}^{C V}\right. \\
& \left.\left.\left.\left.+\operatorname{slope}_{i j}^{t} \times \sum_{m} \bar{U}_{m} \times\left(D T_{i j c k t m}^{C V}-L T_{t} \times x_{i j c k t m}^{C V}\right)\right)\right)\right]\right)+ \\
& C^{L}\left(\sum_{c \in C^{E V}} \sum_{k \in S_{c}^{E V}} a t_{N+1 c k}^{E V}+\sum_{c \in C} \sum_{k \in S_{c}^{C V}} a t_{N+1 c k}^{C V}\right)+ \\
& P B\left(\sum_{i \in V_{0}} \sum_{j \in F^{\prime}} \sum_{c \in C^{E V}} \sum_{k \in S_{c}^{E V}} \sum_{t \in T} \sum_{m \in M} x_{i j c k t m}^{E V}\right)
\end{aligned}
$$

The first and second row of the objective function are the vehicle purchase costs. $F_{C}^{E V}$ and $F_{C}^{C V}$ are the fixed cost of ECV and ICCV per day of operation and their value is estimated using equation 3.9.

The fuel energy consumed by ICCVs is minimized in rows 3 to 6 followed by the electric energy consumption of ECVs in rows 7 to 10. As it was explained before, when the travel times are constant the energy consumption of a vehicle can be estimated as $\left[\left(\propto_{i j} \cdot W+\beta_{i j}\right) \times t t_{i j} \times x_{i j}+\left(\propto_{i j} \cdot U_{i j}\right) \times t t_{i j}\right]$, where $U_{i j}$ was forced to be zero when $x_{i j}$ is zero. 
Having the travel time on arc $(i, j), t_{i j}$, estimated as $\left[\right.$ intercept $_{i j}^{t}+$ slope $\left.t_{i j}^{t} \times\left(D T_{t}-L T_{t}\right)\right]$ and substituting it with $t_{i j}$ in the vehicle energy consumption equation, the energy consumption function becomes non-linear due to multiplying two decision variables shown in red:

$$
\begin{aligned}
\left(\propto_{i j} . W+\beta_{i j}\right) \times\left(\text { intercept }_{i j}^{t}+\text { slope }_{i j}^{t} \times\left(D T_{i}-L T_{t}\right)\right) \times x_{i j t} \\
+\left(\propto_{i j} . U_{i j}\right) \times\left(\text { intercept }_{i j}^{t}+\text { slope }_{i j}^{t} \times\left(D T_{i}-L T_{t}\right)\right)
\end{aligned}
$$

In order to make the above function linear, the variable $D T_{i j t m}$ is defined. This variable shows the departure time from node $i$ to node $j$ at time interval $t$ and load interval $m$. Using this variable the energy consumption function becomes linear as shown below and in the objective function.

$$
\begin{aligned}
& \left(\propto_{i j} \cdot W+\beta_{i j}\right) \times\left(\text { intercept }_{i j}^{t} \times x_{i j t m}+\text { slope }_{i j}^{t} \times\left(D T_{i j t m}-L T_{t} \times x_{i j t m}\right)\right)+ \\
& \left(\propto_{i j} \cdot \bar{U}_{m}\right) \times\left(\text { intercept }_{i j}^{t} \times x_{i j t m}+\text { slope }_{i j}^{t} \times\left(D T_{i j t m}-L T_{t} \times x_{i j t m}\right)\right)
\end{aligned}
$$

The eleventh row of objective function minimizes the LEZ penalty cost imposed if any ICCV enters any LEZ zone in the network.

The user inconvenience cost is minimized in the twelfth and thirteenth rows of the objective function in the form of waiting or delayed service cost penalties. All demand 
nodes have desired service time windows. Whenever the service is delayed or is started earlier than the customer's desired time, a penalty is incurred.

Row fourteen tries to minimize the emission cost by decreasing the cost of emission produced more than the limit or by increasing the amount of extra emission credit to be sold to other companies.

The labor cost is minimized in the fifteenth row. The final row minimizes the battery degradation cost which is calculated as the multiplication of the battery cycle cost by the number of times the battery of ECV is replaced at a charging station. The battery cycle cost is estimated by dividing the cost of battery by the number of times it can be fully charged in its life cycle.

\subsubsection{Constraints}

In this subsection the problem constraints are provided and explained. Constraint 4.3 ensures that each demand node has exactly one successor.

$$
\begin{gathered}
\sum_{c \in C_{E V}} \sum_{k \in K_{E V}} \sum_{j \in V_{N+1}^{\prime}} \sum_{t \in T} \sum_{m \in M} x_{i j c k t m}^{E V}+\sum_{c \in C_{C V}} \sum_{k \in K_{C V}} \sum_{j \in V_{N+1}} \sum_{t \in T} \sum_{m \in M} x_{i j c k t m}^{C V}=1 \\
\in V, i \neq j
\end{gathered}
$$

Constraints 4.4 and 4.5 guarantee that for each node in the network the number of incoming arcs is equal to the number of outgoing arcs for each vehicle type. 
$\sum_{j \in V^{\prime}} \sum_{t \in T} \sum_{m \in M} x_{i j c k t m}^{E V}$

$-\sum_{j \in V^{\prime}} \sum_{t \in T} \sum_{m \in M} x_{j i c k t m}^{E V}=0 \quad \forall i \in V^{\prime}, c \in C_{E V}, k \in K_{E V}, i \neq j$

$\sum_{j \in V_{N+1}} \sum_{t \in T} \sum_{m \in M} x_{i j c k t m}^{C V}$

$-\sum_{j \in V_{0}} \sum_{t \in T} \sum_{m \in M} x_{j i c k t m}^{C V}=0 \quad \forall i \in V, c \in C_{C V}, k \in K_{C V}, i \neq j$

Constraints 4.6 and 4.7 force each vehicle to be assigned to a maximum of one route.

$\sum_{j \in V^{\prime}} \sum_{t \in T} \sum_{m \in M} x_{0 j c k t m}^{E V} \leq 1 \quad \forall c \in C_{E V}, k \in K_{E V}$

$\sum_{j \in V} \sum_{t \in T} \sum_{m \in M} x_{0 j c k t m}^{C V} \leq 1 \quad \forall c \in C_{C V}, k \in K_{C V}$

$F^{\prime}$ is a set of dummy nodes representing visits to each vertex in the set of charging stations, $F$. Constraint 4.8 ensures that each node in the set $F^{\prime}$ is visited at most once by each vehicle. This makes it possible for each charging station to be visited once, multiple times or not at all by the ECVs on the road.

$\sum_{j \in V_{n+1}} \sum_{t \in T} \sum_{m \in M} x_{i j c k t m}^{E V} \leq 1 \quad \forall c \in C_{E V}, k \in K_{E V}, \forall i \in F^{\prime}, i \neq j$ 
Travel times on arcs are linked through constraints 4.9 and 4.10, which ensure the connectivity of travel times on the traveled arcs by each vehicle.

$$
\begin{aligned}
& a t_{j c k}^{E V} \geq a t_{i c k}^{E V}+S_{i} \sum_{t \in T} \sum_{m \in M} x_{i j c k t m}^{E V}+\sum_{t \in T} \sum_{m \in M} i n t e r c e p t_{i j}^{t} \times x_{i j c k t m}^{E V} \\
& +\sum_{t \in T} \sum_{m \in M} S l o p e_{i j}^{t} \times\left(D T_{i j c k t m}^{E V}-L T_{t-1} \times x_{i j c k t m}^{E V}\right) \\
& -T \cdot\left(1-\sum_{t} \sum_{m} x_{i j c k t m}^{E V}\right) \quad \forall c \in C_{E V}, k \in K_{E V}, i \in V^{\prime}{ }_{0}, j \in V^{\prime}{ }_{n+1}, i \\
& \neq j \\
& a t_{j c k}^{C V} \geq a t_{i c k}^{C V}+S_{i} \sum_{t \in T} \sum_{m \in M} x_{i j c k t m}^{C V}+\sum_{t \in T} \sum_{m \in M} \text { intercept }_{i j}^{t} \times x_{i j c k t m}^{C V} \\
& +\sum_{t \in T} \sum_{m \in M} S l o p e_{i j}^{t} \times\left(D T_{i j c k t m}^{C V}-L T_{t-1} \times x_{i j c k t m}^{C V}\right) \\
& -T .\left(1-\sum_{t} \sum_{m} x_{i j c k t m}^{C V}\right) \quad \forall c \in C_{C V}, k \in K_{C V}, i \in V_{0}, j \in V_{n+1}, i \\
& \neq j
\end{aligned}
$$

These equations are extension of the equations 3.17 and 3.18 for the case of TimeDependent GVRP. Basically arrival time at node $\mathrm{j}$ for an ECV can be formulated as:

$$
\begin{aligned}
& a t_{j c k}^{E V} \geq a t_{i c k}^{E V}+\left(t t_{i j}^{t}+S_{i}\right) \cdot \sum_{t} \sum_{m} x_{i j c k t m}^{E V} T .\left(1-\sum_{t} \sum_{m} x_{i j c k t m}^{E V}\right) \\
& \forall c \in C_{E V}, k \in K_{E V}, i \in V^{\prime}{ }_{0}, j \in V^{\prime}{ }_{n+1}, i \neq j
\end{aligned}
$$

where, $t t_{i j}^{t}$ is the travel time on arc $(i, j)$ and is calculated as: 
$t t_{i j}^{t}=$ intercept $_{i j}^{t}+\operatorname{Slope}_{i j}^{t} \times\left(d t_{i c k}^{E V}-L T_{t}\right)$

Since $d t_{i c k}^{E V}$ is a decision variable, multiplying $t t_{i j}^{t}$ by $x_{i j c k t m}^{E V}$ makes the problem nonlinear. In order to deal with nonlinearity and make the problem linear, the part in the box in equation 4.11 is substituted with the below function resulting in constraints 4.9 and 4.10.

$$
\begin{aligned}
S_{i} \sum_{t \in T} \sum_{m \in M} x_{i j c k t m}^{E V}+\sum_{t \in T} \sum_{m \in M} \text { intercept }_{i j}^{t} \times x_{i j c k t m}^{E V} & \\
& +\sum_{t \in T} \sum_{m \in M} S_{\text {lope }}^{t} \times\left(D T_{i j c k t m}^{E V}-L T_{t-1} \times x_{i j c k t m}^{E V}\right)
\end{aligned}
$$

Departure time from each node in the network is calculated through constraints 4.13 and 4.14 The estimated departure time is then used to estimate the decision variables $D T_{i j c k t m}^{C V}$ and $D T_{i j c k t m}^{E V}$ as shown in constraints 4.15 to 4.18 .

$$
\begin{aligned}
& d t_{i c k}^{E V} \geq a t_{i c k}^{E V}+S_{i} \cdot \sum_{j \in V^{\prime}{ }_{N+1}} \sum_{t} \sum_{m} x_{i j c k t m}^{E V} \quad \forall c \in C_{E V}, k \in K_{E V}, i \in V^{\prime} \\
& d t_{i c k}^{C V} \geq a t_{i c k}^{C V}+S_{i} \cdot \sum_{j \in V_{N+1}} \sum_{t} \sum_{m} x_{i j c k t m}^{C V} \quad \forall c \in C_{C V}, k \in K_{C V}, i \in V \\
& D T_{i j c k t m}^{E V V} \leq M x_{i j c k t m}^{E V} \quad \forall c \in C_{E V}, k \in K_{E V}, i \in V^{\prime}, j \in V_{N+1}^{\prime}, t \in T, m \in M \\
& \sum_{j \in V_{N+1}} \sum_{t} \sum_{m} D T_{i j c k t m}^{E V}=d t_{i c k}^{E V} \quad \forall c \in C_{E V}, k \in K_{E V}, i \in V^{\prime} \\
& D T_{i j c k t m}^{C V} \leq M x_{i j c k t m}^{C V} \quad \forall c \in C_{C V}, k \in K_{C V}, i \in V, j \in V_{n+1}, t \in T, m \in M
\end{aligned}
$$




$$
\sum_{j \in V_{N+1}} \sum_{t} \sum_{m} D T_{i j c k t m}^{C V}=d t_{i c k}^{C V} \quad \forall c \in C_{C V}, k \in K_{C V}, i \in V
$$

Constraints 4.19 to 4.22 guarantee that the arrival time and departure time of vehicles at each node are within the daily delivery operations working hour $\left[T_{0} \mathrm{~T}\right]$.

$$
\begin{array}{ll}
T_{0} \leq a t_{j c k}^{E V} \leq T & \forall c \in C_{E V}, k \in K_{E V}, \forall j \in V^{\prime}{ }_{n+1} \\
T_{0} \leq a t_{j c k}^{C V} \leq T & \forall c \in C_{C V}, k \in K_{C V}, \forall j \in V_{n+1} \\
T_{0} \leq d t_{j c k}^{E V} \leq T & \forall c \in C_{E V}, k \in K_{E V}, \forall j \in V^{\prime}{ }_{n+1} \\
T_{0} \leq d t_{j c k}^{C V} \leq T & \forall c \in C_{C V}, k \in K_{C V}, \forall j \in V_{n+1}
\end{array}
$$

Constraints 4.23 to 4.26 link the departure time with the corresponding time window $t$ so that the proper travel time function is used to estimate the travel time.

$$
\begin{gathered}
d t_{i c k}^{C V}-U T_{t} \leq 100\left(1-\sum_{m} x_{i j c k t m}^{C V}\right) \\
\forall c \in C_{C V}, k \in K_{C V}, i \in V_{0}, j \in V_{n+1}, t \in T \\
d t_{i c k}^{C V} \geq L T_{t} \sum_{m} x_{i j c k t m}^{C V} \quad \forall c \in C_{C V}, k \in K_{C V}, i \in V_{0}, j \in V_{n+1}, t \in T \\
d t_{i c k}^{E V}-U T_{t} \leq 100\left(1-\sum_{m} x_{i j c k t m}^{E V}\right)
\end{gathered}
$$

$$
\forall c \in C_{E V}, k \in K_{E V}, i \in V^{\prime}{ }_{0}, j \in V^{\prime}{ }_{n+1}, t \in T
$$


$d t_{i c k}^{E V} \geq L T_{t} \sum_{m} x_{i j c k t m}^{E V} \quad \forall c \in C_{E V}, k \in K_{E V}, i \in V^{\prime}{ }_{0}, j \in V^{\prime}{ }_{n+1}, t \in T$

If the arrival of a vehicle at a demand node is not within the desired delivery time window, constraints 4.27 to 4.30 calculate the early or delayed service time at the demand node.

$$
\begin{array}{ll}
w_{j c k}^{E V} \geq e_{j}-a t_{j c k}^{E V} & \forall c \in C_{E V}, k \in K_{E V}, \forall j \in V \\
d_{j c k}^{E V} \geq a t_{j c k}^{E V}-l_{j} & \forall c \in C_{E V}, k \in K_{E V}, \forall j \in V \\
w_{j c k}^{C V} \geq e_{j}-a t_{j c k}^{C V} & \forall c \in C_{C V}, k \in K_{C V}, \forall j \in V \\
d_{j c k}^{C V} \geq a t_{j c k}^{C V}-l_{j} & \forall c \in C_{C V}, k \in K_{C V}, \forall j \in V
\end{array}
$$

The total load a vehicle carries is limited by its capacity through constraints 4.31 to 4.32 . Constraints 4.33 and 4.34 force the load of a vehicle over arc $(i, j)$ to be zero if that vehicle does not traverse the arc.

$$
\begin{gathered}
\sum_{i \in V} q_{i} \sum_{j \in V_{N+1}} \sum_{t} \sum_{m} x_{i j c k t m}^{E V} \leq Q_{c}^{E V} \quad \forall c \in C_{E V}, k \in K_{E V} \\
\sum_{i \in V} q_{i} \sum_{j \in V_{N+1}} \sum_{t} \sum_{m} x_{i j c k t m}^{C V} \leq Q_{c}^{C V} \quad \forall c \in C_{C V}, k \in K_{C V} \\
q_{j} \cdot \sum_{t} \sum_{m} x_{i j c k t m}^{E V} \leq U_{i j c k}^{E V} \leq\left(Q_{c}^{E V}-q_{i}\right) \cdot \sum_{t} \sum_{m} x_{i j c k t m}^{E V} \\
\forall c \in C_{E V}, k \in K_{E V}, i \in V_{0}, j \in V_{n+1} \\
q_{j} \cdot \sum_{t} \sum_{m} x_{i j c k t m}^{C V} \leq U_{i j c k}^{C V} \leq\left(Q_{c}^{C V}-q_{i}\right) . \sum_{t} \sum_{m} x_{i j c k t m}^{C V} \\
\forall c \in C_{C V}, k \in K_{C V}, i \in V_{0}, j \in V_{n+1}
\end{gathered}
$$


Balance of load flow at each node is defined through constraints 4.35 and 4.36. These constraints model the vehicle load flow as increasing by the amount of cargo demand of each visited demand node.

$$
\begin{gathered}
\sum_{j \in V^{\prime}} U_{j i c k}^{E V}-\sum_{j \in V^{\prime}{ }_{N+1}} U_{i j c k}^{E V}=q_{i} \sum_{j \in V^{\prime}} \sum_{N+1} \sum_{m} x_{i j c k t m}^{E V} \\
\forall c \in C_{E V}, k \in K_{E V}, \forall i \in V \\
\sum_{j \in V_{0}} U_{j i c k}^{C V}-\sum_{j \in V_{N+1}} U_{i j c k}^{C V}=q_{i} \sum_{j \in V_{N+1}} \sum_{t} \sum_{m} x_{i j c k t m}^{E V} \\
\forall c \in C_{E V}, k \in K_{E V}, \forall i \in V
\end{gathered}
$$

Constraints 4.37 to 4.40 link the vehicle load on each arc with the corresponding load interval $m$ so that the proper load interval is used while estimating the vehicle energy requirements along each arc.

$$
\begin{gathered}
U_{j i c k}^{C V} \geq L U_{m} \sum_{t} x_{i j c k t m}^{C V} \quad \forall c \in C_{C V}, k \in K_{C V}, i \in V_{0}, j \in V_{n+1}, m \in M \\
U_{j i c k}^{C V}-U U_{m} \leq M\left(1-\sum_{t} x_{i j c k t m}^{C V}\right) \\
\forall c \in C_{C V}, k \in K_{C V}, i \in V_{0}, j \in V_{n+1}, m \in M \\
U_{j i c k}^{E V} \geq L U_{m} \sum_{t} x_{i j c k t m}^{E V} \quad \forall c \in C_{E V}, k \in K_{E V}, i \in V_{0}^{\prime}, j \in V^{\prime}{ }_{n+1}^{\prime}
\end{gathered}
$$




$$
\begin{aligned}
& U_{j i c k}^{E V}-U U_{m} \leq M\left(1-\sum_{t} x_{i j c k t m}^{E V}\right) \\
& \forall c \in C_{E V}, k \in K_{E V}, i \in V_{0}^{\prime}, j \in V^{\prime}{ }_{n+1}, m \in M
\end{aligned}
$$

In constraint 4.41 , the remaining battery capacity of all electric vehicles are set to their full battery capacity before starting their route. This means that all the vehicles leave the depot with fully charged batteries.

$$
R_{o c k=B C_{c}^{E V}} \quad \forall c \in C_{E V}, k \in K_{E V}
$$

Constraints 4.42 set the battery level of a vehicle arriving at a node succeeding a demand node in accordance with the energy consumption on the arc joining these two nodes. Constraints 4.43 define the same relation for the nodes succeeding a charging station.

$$
\begin{aligned}
R_{j c k} \leq R_{i c k}- & \left(\propto^{\prime}{ }_{i j} \times w_{c}^{E V}+\beta^{\prime}{ }_{i j}\right) \times\left(\text { intercept }_{i j}^{t} \times \sum_{m} x_{i j c k t m}^{E V}\right. \\
& \left.+\operatorname{slope}_{i j}^{t} \times \sum_{m}\left(D T_{i j c k t m}^{E V}-L T_{t} \times x_{i j c k t m}^{E V}\right)\right) \\
& +\propto^{\prime}{ }_{i j} \times\left(\text { intercept } t_{i j}^{t} \times \sum_{m} M_{a v g} \times x_{i j c k t m}^{E V}\right. \\
& \left.+\operatorname{slope}_{i j}^{t} \times \sum_{m} \bar{U}_{m} \times\left(D T_{i j c k t m}^{E V}-L T_{t} \times x_{i j c k t m}^{E V}\right)\right)+E C_{k}(1 \\
& \left.-\sum_{m} x_{i j c k t m}^{E V}\right)
\end{aligned}
$$

$$
\forall c \in C_{E V}, k \in K_{E V}, \forall i \in V, j \in V^{\prime}{ }_{n+1}, i \neq j, \forall t \in T
$$




$$
\begin{aligned}
R_{j c k} \leq E C_{k}- & \left(\propto^{\prime}{ }_{i j} \times w_{c}^{E V}+\beta^{\prime}{ }_{i j}\right) \times\left(\text { intercept }_{i j}^{t} \times \sum_{m} x_{i j c k t m}^{E V}\right. \\
& \left.+\operatorname{slope}_{i j}^{t} \times \sum_{m}\left(D T_{i j c k t m}^{E V}-L T_{t} \times x_{i j c k t m}^{E V}\right)\right) \\
& +\propto^{\prime}{ }_{i j} \times\left(\text { intercept } i_{i j}^{t} \times \sum_{m} M_{\text {avg }} \times x_{i j c k t m}^{E V}\right. \\
& \left.+ \text { slope }_{i j}^{t} \times \sum_{m} \bar{U}_{m} \times\left(D T_{i j c k t m}^{E V}-L T_{t} \times x_{i j c k t m}^{E V}\right)\right)
\end{aligned}
$$

$$
\forall c \in C_{E V}, k \in K_{E V}, \forall i \in F^{\prime} \cup\{0\}, j \in V^{\prime}{ }_{n+1}, i \neq j, \forall t \in T
$$

Constraint 4.44 ensure that if an internal combustion engine vehicle visits any demand node in a Low Emission Zone, the decision variable, $y_{c k l}^{C V}$, is set to one for the vehicle and the corresponding zone. Finally, binary decision variables are defined in constraints 4.45 .

$$
\begin{aligned}
& \sum_{i \in l} \sum_{j \in V_{N+1}} \sum_{t} \sum_{m} x_{i j c k t m}^{C V} \leq 100 y_{c k l}^{C V} \quad \forall c \in C_{C V}, k \in K_{C V}, \in L E Z_{l} \\
& x_{i j c k t m}^{E V}, x_{i j c k t m}^{C V}, y_{c k l}^{C V} \in\{0,1\}
\end{aligned}
$$

\subsection{Numerical Study}

In this section the developed mathematical formulation for the defined Time-Dependent GVRP is solved for a small size problem. A small network of 6 demand nodes with one LEZ, which includes demand nodes $\mathrm{P}_{3}, \mathrm{P}_{5}, \mathrm{P}_{6}$ and one charging station, is defined as shown in Figure 4.4. The LEZ penalty, carbon permit, and emission cap is assumed to be \$100/day, \$0.50/gram, and 20 grams respectively. 
First it is assumed that travel time on all arcs in the network is constant over the planning period and the formulation developed for the GVRP is used to solve the problem to optimality. Then, the planning period is divided into three time windows and different travel time functions are defined for each direction of arcs in the network to account for different levels of congestion. The developed mathematical model for the defined TDGVRP is used to solve the problem to optimality. Figure 4.5 and Figure 4.6 show the solution to GVRP and Time-Dependent GVRP respectively.

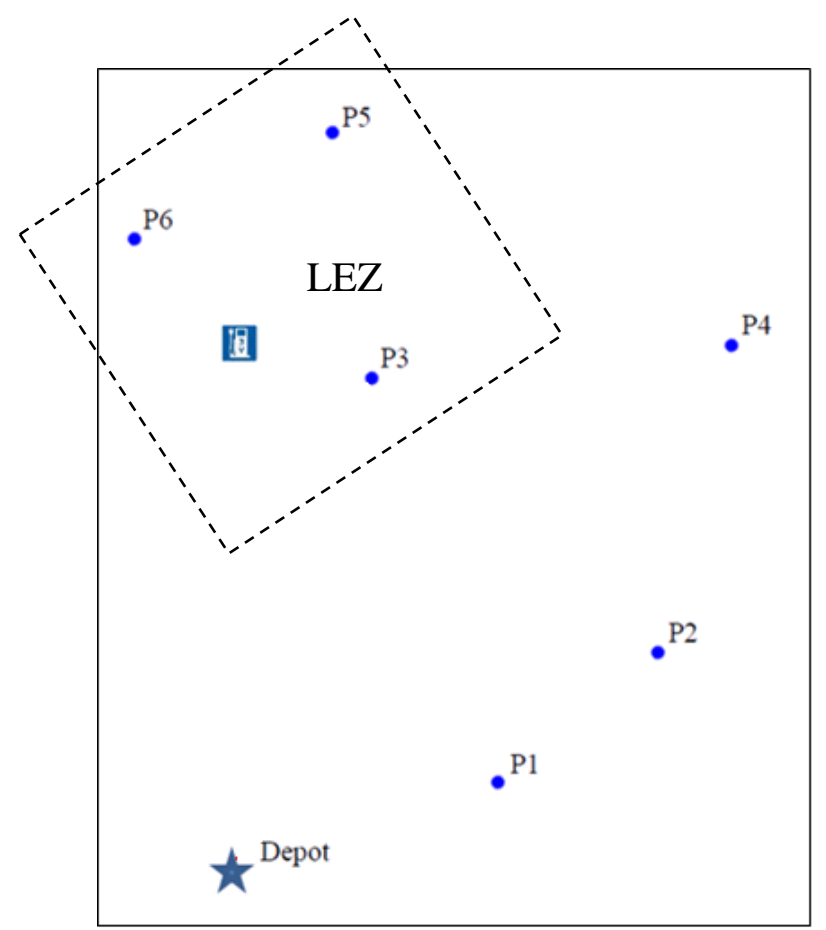

Figure 4. 4 Network of the Numerical case Study - 6 Demand Nodes

In both figures the green and black lines indicate that the route is served by ECV and ICCV respectively. The result show that in the both cases of static and TimeDependent travel times the optimal fleet size is the same, however, there are some changes 
in the routing of the vehicles in order to avoid congestion. The optimal route for the ECV when the travel time on arcs is constant is: Depot $\rightarrow P_{3} \rightarrow P_{5} \rightarrow C S \rightarrow P_{6}$. When the travel time on arcs changes over the planning period due to congestion, the optimal route for ECV is changed to: Depot $\rightarrow P_{3} \rightarrow C S \rightarrow P_{6} \rightarrow P_{5}$. In fact when the ECV arrives to $P_{3}$, its battery capacity is not enough to visit $P_{5}$, or $P_{6}$ due to the high congestion on the arcs. Therefore, it first visits the CS to recharge its battery, and then it goes to $P_{6}$ to avoid congestion on $\left(\mathrm{CS}, P_{5}\right)$. From $P_{6}$ it goes to $P_{5}$ and then returns to Depot. In the case of the ICCV route, the sequence of the demand points is reversed when the time dependency of travel times is accounted for. First, the vehicle visits demand point $P_{4}$ to avoid congestion on the routes to $P_{1}$ and $P_{2}$ in the morning, and then goes to $P_{2}$ followed by $P_{1}$ and then returns to Depot.

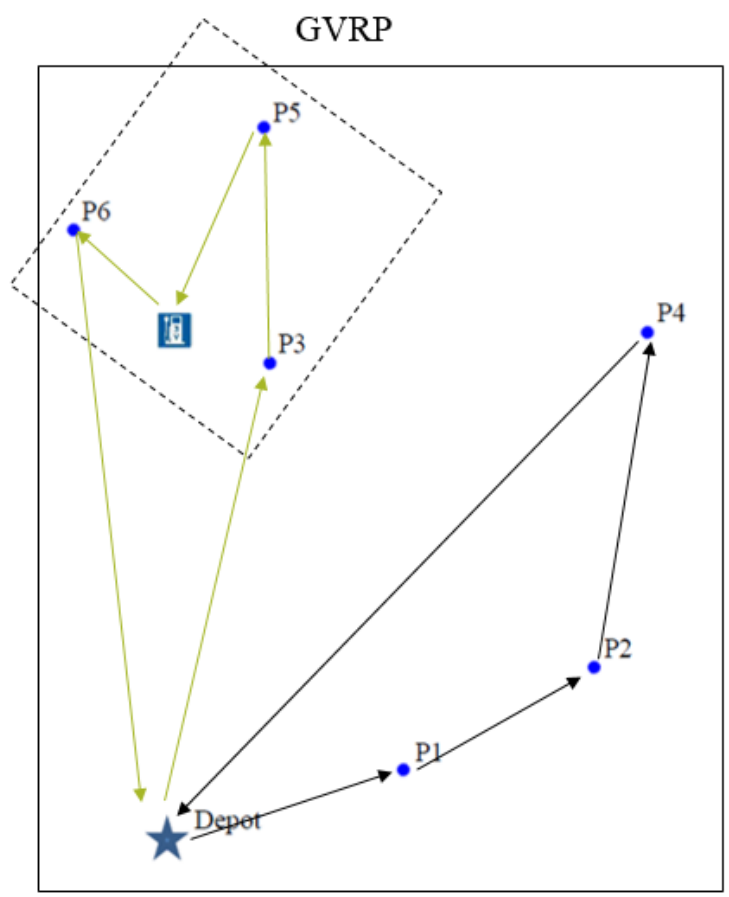

Figure 4. 5 Small Size Network__ GVRP Result 


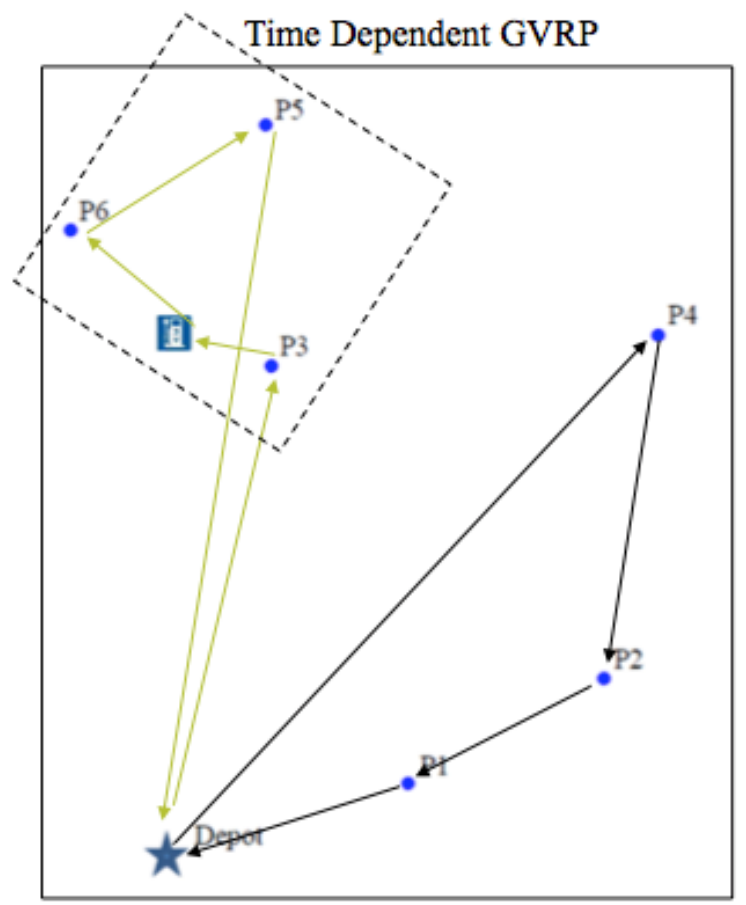

Figure 4. 6 Small Size Network - TDGVRP Result

\section{$\underline{4.5 \text { Summary }}$}

In this chapter the problem characteristics were explained. The assumptions used in the research were introduced and the developed mathematical model was presented with a brief explanation of the objective function and constraints. The developed mathematical model for the TDGVRP was solved for a small size problem using the Xpress commercial solver. The result of the model was compared with the results of GVRP for the case of static travel times and the differences were highlighted. 


\section{Chapter 5}

\section{HEURISTIC METHOD}

It is well known that VRP is an NP-hard problem and as the size of the problem grows commercial solvers like Xpress become unable to find optimal solutions to the problem. Therefore, it is essential to develop an efficient heuristic algorithm that can find sound solutions in a reasonable amount of time. In this chapter, the heuristic algorithm developed to solve the Time-Dependent GVRP defined in this dissertation is introduced and explained in detail. Then the quality of heuristic solutions is verified by comparing the heuristic solutions with the exact solutions to the problem found using Xpress solver on small size problems.

\subsection{Overall Explanation of the Heuristic Algorithm}

The heuristic developed in this study is based on the Ruin and Recreate (RR) approach, a new class of algorithms introduced by Schrimpf et al. (2000) used to solve VRPTW instances. The basic idea behind the developed algorithm is to obtain new solutions by deconstructing an existing feasible solution, and then rebuilding it by following a set of procedures to obtain a new complete solution. Overall, the proposed heuristic algorithm is comprised of four main components:

- A constructive heuristic used to: $a$ ) build initial feasible solutions to the problem and $b$ ) to build complete solutions from deconstructed partial solutions. 
- A ruin strategy for selecting a part of the current solution and remove it to form partial solutions.

- A decision rule to be used as a criterion for solution acceptance. If the new solution is accepted it becomes the current solution and the ruin strategy is applied on the new current solution in the next iteration. On the other hand, if the new solution is not accepted, the ruin strategy will be applied to the previous solution until a new solution is accepted.

- Stopping criteria used to stop the algorithm and accept the best solution as a final solution to the problem.

According to the description above, the heuristic algorithm proposed in this dissertation, builds an initial feasible solution and then performs a set of iterations on an existing current solution until some stopping criteria is met. At each iteration the current best solution is deconstructed and rebuilt a number of times until a better solution is found and used as a new current solution. Indeed, many partial solutions are obtained from the same current solution by removing a proper set of customers and completing the resulting solution according to a recreate strategy.

The proposed algorithm shares some similarities with the classic local search (LS) approach, but it presents some advantages that lead to feasible solutions of better quality. While both of the methods make use of a systematic perturbation of the current solution that leads to a new solution, they are based on different concepts of neighborhood. In the LS approach, a new solution is achieved by a small modification to the current solution 
such as movement of a customer to a different route. In fact, LS performs a deep evaluation of solutions close to the current one and chooses a better neighboring solution. However, the algorithm proposed in this dissertation generates new solution by deconstructing a larger part of the current solution. Therefore, our algorithm not only relies on exploring solutions close to the current one, but also evaluates solutions that might be far from the current one in the feasible solution space. Looking into solutions far from the current one, which is called diversification strategy, is rarely applied during evolution of search in the LS algorithms.

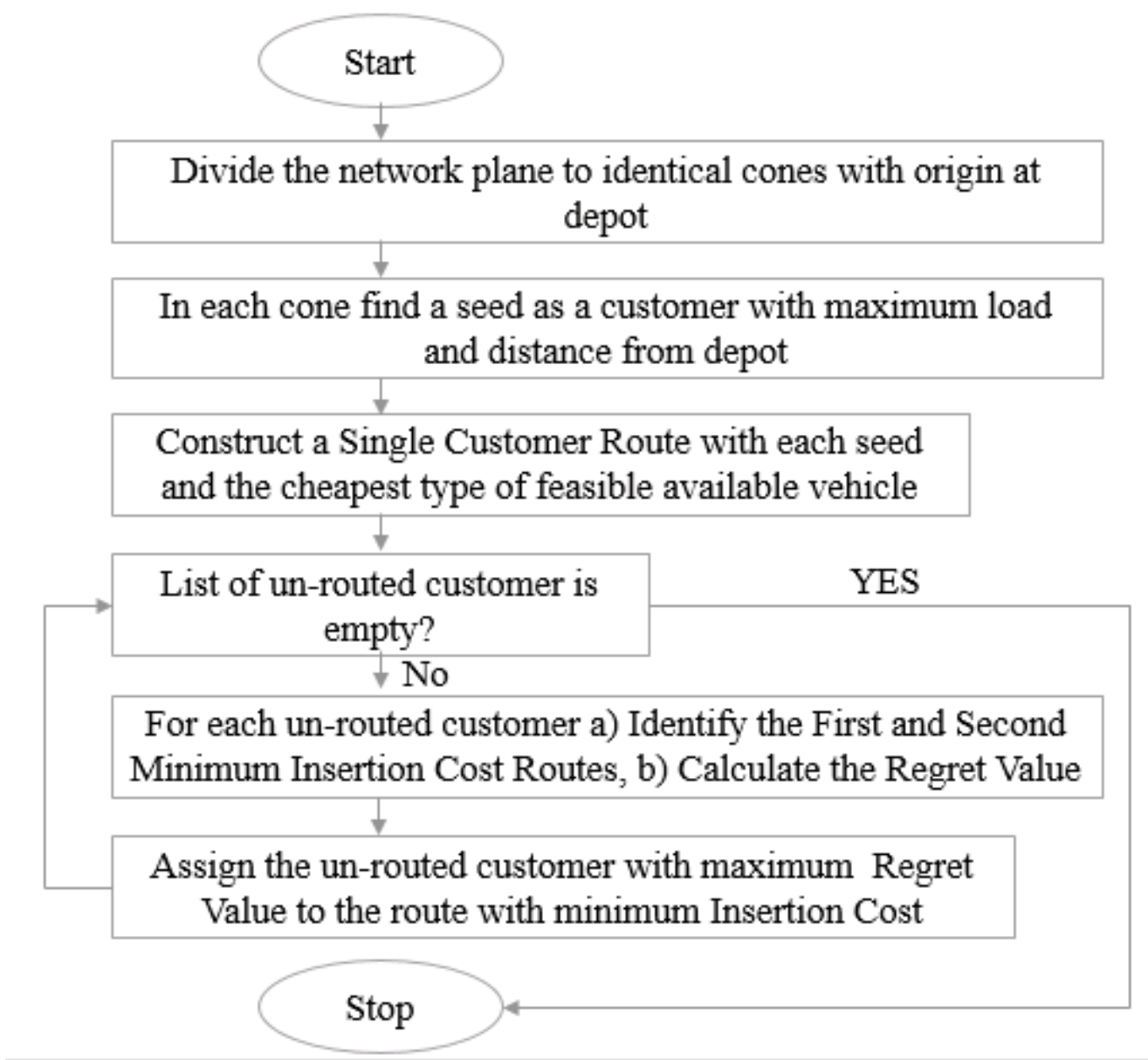

Figure 5. 1 Constructive Heuristic Work Flow 
Figure 5.1 and Figure 5.2 show the flow chart of the heuristic algorithm proposed in this dissertation and coded in Python language. As seen in these figures, two sets of procedures are followed. First an initial feasible solution is generated using a constructive heuristic approach shown in Figure 5.1. Then, as shown in Figure 5.2, the generated solution is improved by deconstructing and rebuilding new solutions from the current solution until some stopping criteria are met. The steps in both procedures are explained in details in the following sections of this chapter.

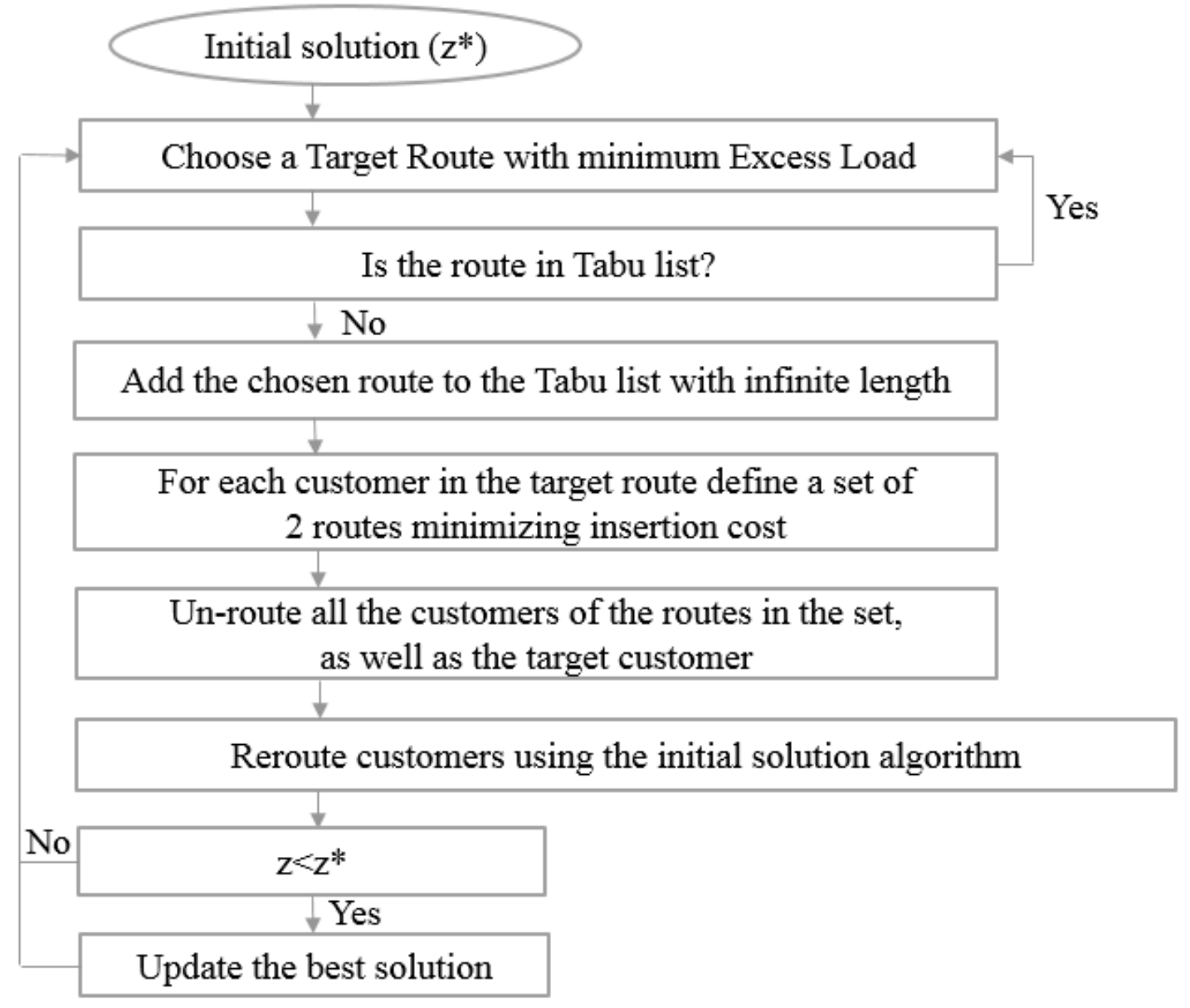

Figure 5. 2 Improvement Heuristic Work Flow 


\subsection{Constructive Heuristic Algorithm}

The constructive heuristic algorithm proposed in this study is based on the regret procedure presented in Christofides, Mingozzi, and Toth (1979) and Potvin and Rousseau (1993).

The algorithm starts with generating a partial solution comprised of a set of feasible single-customer routes. To generate the partial solution, first the network plane is divided into a number of identical cones. Each cone has an origin at depot. Figure 5.3 shows the network of 10 customer demand nodes divided into 4 identical cones with an angle of $45^{\circ}$. The number of cones is calculated as the sum of customer demands multiplied by 1.5 and divided by the maximum capacity of available vehicles. Then, in each cone one demand point is selected as a seed. To select the seeds, one cone at a time is considered and for each demand node in the cone a weight value is estimated using the equation 5.1. As it can be seen in this equation, the weights are calculated as a function of demand and distance from depot. Once the weights are calculated for each demand node, the node with the maximum weight value is chosen as the cone's seed. In fact seeds are chosen as the demand nodes with the combination of farthest distance from the depot and the highest demand. Having found a seed in each cone, single-customer routes are generated for each seed. Also a feasible minimum cost vehicle is assigned to each of the defined routes.

$W D_{i}=q_{i}+d_{o i}$ 
where,

- $W D_{i}=$ Weight of the customer $i$,

- $q_{i}=$ Demand of the customer $i$,

- $\quad$ and $d_{o i}=$ Distance between the depot and customer $i$.

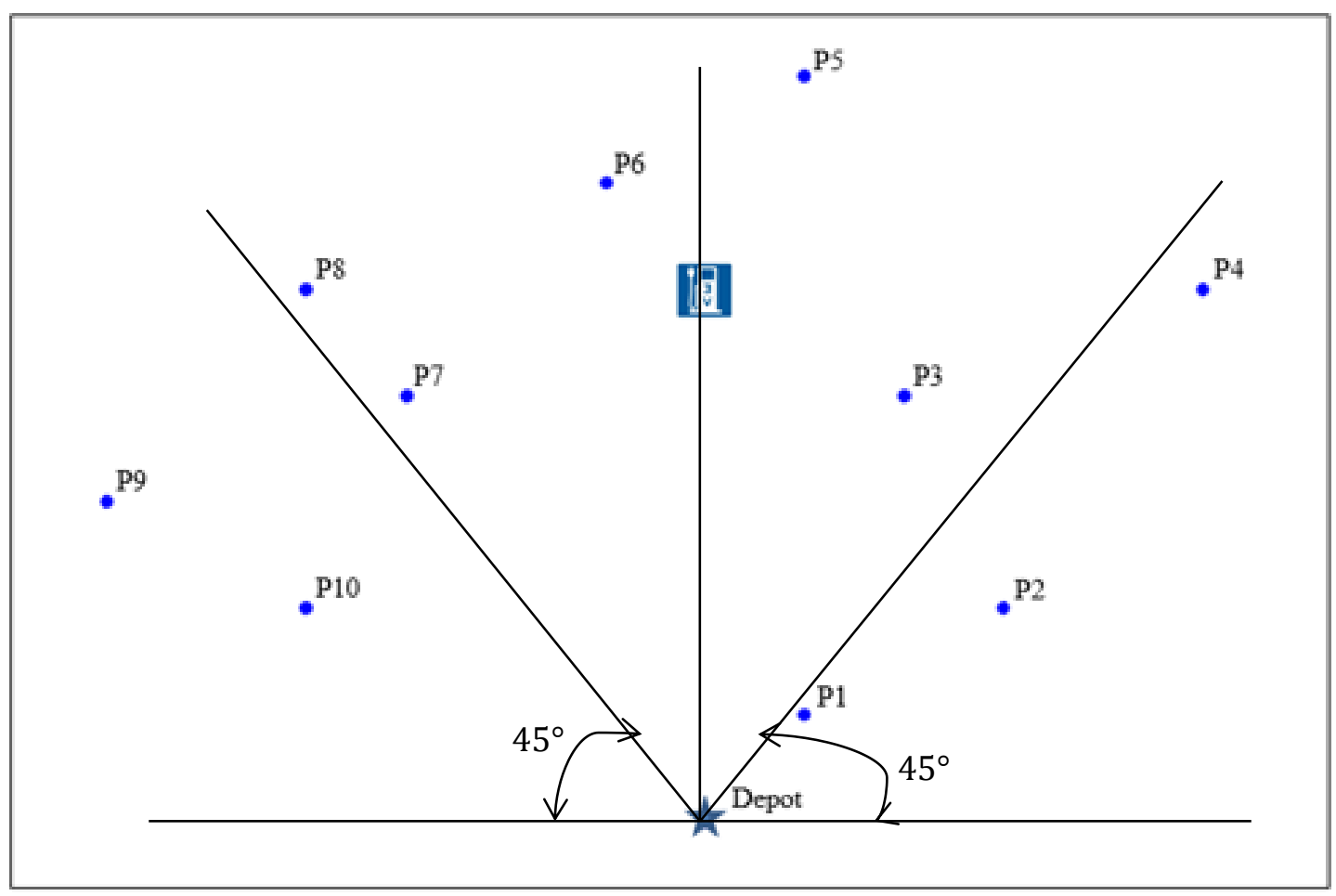

Figure 5. 3 Sample of identical cones in a network

The set of single customer routes define the partial solution for the constructive phase. The partial solution is completed by iteratively assigning each un-routed demand point either to the existing routes or to a new one. The un-routed customers are chosen and assigned to the routes based on a regret score, $\delta(i)$, which accounts for the urgency of assigning customer $i$ to a route. The computation of $\delta(i)$ is based on a penalty, $\rho_{r}(i)$, 
associated with the cost of assigning customer $i$ to route $r$. This penalty is comprised of six components and is calculated using equation 5.2. In this equation, $\operatorname{Set}_{r}(i)$ denote the set of all possible insertion points for customer $i$ in route $r$. In fact, the penalty of inserting customer $i$ in route $r$ is calculated for a) all possible insertion points in the $\operatorname{Set}_{r}(i)$ and b) for the two types of vehicles, ICCV and ECV, then, the minimum penalty is assigned to $\rho_{r}(i)$

$$
\begin{aligned}
& \rho_{r}(i)=\min _{k \in\{I C C V, E C V\}}\left\{\min _{p \in \operatorname{Set}_{r}(i)}\{\text { extra_energy_req } r(i, p, k)\right. \\
& +v e \text { _ch }_{-} \operatorname{cost}{ }_{r}(i, k)+\text { time_window_pen }_{r}(i, p, k) \\
& +L E Z_{\text {pen }_{r}}(i, p, k)+\text { bat_deg }_{r}(i, p, k)+\text { carbon_permit }(i, p, k) \\
& + \text { extra_Labor_Cost }(i, p, k)\}\}
\end{aligned}
$$

The first component of equation 5.2 is the extra energy required by the vehicle to travel to and from the node after its insertion in the route. The second component is the vehicle change cost if the vehicle serving route $r$ must be changed to visit customer $i$. The third component is the time window penalty if the insertion of customer $i$ to the route $r$ results in service time window violations at any demand nodes. The fourth component is the LEZ penalty cost that incurs when customer $i$ is in LEZ and route $r$ is served by an ICCV. The fourth component is the battery degradation cost, which is applicable only if customer $i$ is inserted in a route served by an electric truck. If the extra energy requirements of the electric truck to serve customer $i$ results in the battery capacity violations, there is a 
need for the battery to be exchanged with a full one and battery degradation cost should be accounted for. The fifth component is the carbon emission cost which is only applicable in the case of ICCVs. And the last one is the extra labor cost due to the increase in travel time after the insertion of the new customer to the route.

\section{Extra Energy Requirement}

The estimation of the variable extra_energy_req $r(i, p, k)$, is slightly different for the two types of vehicle, ICCV and ECV. If $k$ is assumed to be ICCV, then the variable extra_energy_req $r(i, p, k)$ is calculated as the extra energy required by the vehicle to detour to the newly added customer $i$ at insertion point $p$.

For the case of ECVs, if the battery capacity of the vehicle is sufficient to serve the newly added customer $i$, the extra energy required by the vehicle, is estimated as the extra energy consumed to serve the newly added customer $i$ at insertion point $p$. However, if the insertion of customer $i$ to the route $r$ at point $p$ lead to the ECV battery capacity violation, the extra energy required to visit a charging station should be considered as well. In fact, when vehicle energy requirement exceeds the vehicle battery capacity, a charging station should be visited to extend the vehicle driving range by swapping its battery with a fully charged one. The visit to a charging station adds to the vehicle energy requirements.

In order to find the best insertion point for a CS in route $r$, a battery violation penalty is estimated for each possible insertion point as shown in equation 5.3. Then, the best insertion point is identified as the one minimizing the battery violation penalty. There 
might be cases where charging stations are required to be visited more than once. In fact charging station visit nodes are inserted to the route until the value of $\overrightarrow{\gamma_{l}}-B$ cap becomes less than zero for all the nodes in the route.

pen $_{\text {batt }}=\sum_{i \in V(r)} \overrightarrow{\gamma_{l}}-$ Bcap

where,

- $V(r)=$ set of customer demand nodes in route $r$

- $\vec{\gamma}_{l}=$ battery charge that is needed to travel either from the previous visit to a charging station or from the depot to vertex $i$

- Bcap of the vehicle assigned to route $r$

Therefore, if the vehicle's available battery is not sufficient to serve a newly added customer $i$, the variable extra_energy_req ${ }_{r}(i, p, k)$ is estimated as the sum of the extra energy required to serve the newly added customer and the extra energy required to visit charging stations if applicable.

\section{Vehicle Change Cost}

The second component in estimation of $\rho_{r}(i)$ is the vehicle change cost (equation 5.2). Vehicle change cost is experienced when the loading capacity of the current vehicle assigned to the route $r$ is not sufficient to serve the newly added customer. Therefore, the 
vehicle should be changed to the cheapest vehicle of higher capacity. The vehicle change cost is estimated as:

veh_chg_cost ${ }_{r}(i, k)=F C^{\text {curent }}-F C^{\text {new }}$

where,

- $F C^{\text {current }}=$ Fixed cost of current vehicle assigned to route $r$.

- $F C^{\text {new }}=$ Fixed cost of the cheapest available vehicle of type $k$ with higher capacity.

If there is no vehicle of higher capacity available to be assigned to the route, adding customer $i$ to the existing route $r$ make the solution infeasible by violating the vehicle load capacity. Therefore the customer should be assigned to a new route with the cheapest feasible type of vehicle available.

\section{Time Window Penalty}

The time_window_pen ${ }_{r}(i, p, k)$ is the summation of early or delayed service penalties at customer node $i$, and the customers visited after it along the route. The time window penalty is formulated as:

$$
\begin{aligned}
& \text { time_window_pen }_{r}(u)=\sum_{j \epsilon\left\{p^{+}{ }_{i}\right\}} \text { pen }_{\text {early_service }} \times \\
& \max \left(0, \text { earliest_des_serv_time }_{j}-\text { act }_{\text {serv }_{\text {time }} j}\right)+\text { pen }_{\text {delayed }_{\text {service }}} \times \\
& \max \left(0, \text { act }_{\text {serv }_{\text {time }} j}-\text { latest }_{\text {des } \left._{\text {serv }_{\text {time }}}\right)}\right)
\end{aligned}
$$


where,

- $p^{+}=$Set of customer nodes after insertion point $p$ along route $r$

- $\quad$ earliest_des_serv_time $e_{j}=$ Earliest desired service time at customer node $j$

- $\quad$ latest_des_serv_time ${ }_{j}=$ Latest desired service time at customer node $j$

- $a c t \_s e r v_{-}$time $_{j}=$ Actual service time at customer node $j$

\section{$\underline{\text { LEZ Penalty }}$}

The LEZ penalty, $L E Z_{p e n_{r}}(i, p, k)$, is applicable if the customer $i$ is located in a low emission zone and the vehicle type $k$ is ICCV. If the current vehicle assigned to route $r$ is of the type ECV and it passes through LEZ, $\operatorname{LEZ}_{\text {pen }_{r}}(i, p, k=I C C V)$ would have a positive value. On the other hand, if the current vehicle assigned to route $r$ is of the type ICCV and it passes through LEZ, $L E Z_{\text {pen }}(i, p, k=E C V)$ would have a negative value, as the change in the type of the vehicle reduces the routing cost by the amount of LEZ penalty cost.

\section{Battery Degradation Cost}

The battery degradation cost, $b a t_{-} \operatorname{deg}_{r}(i, p, k)$, is applicable if the vehicle type $k$ is ECV and is calculated as:

bat_deg $_{r}(i, p, k=E C V)=\sigma . C_{\text {bat_deg }}$

where, 
- $\sigma=$ Number of Charging Station visits needed to provide the additional energy required to serve customer $i$ by route $r$ at insertion point $p$.

- $C_{\text {bat_deg }}=$ Battery degradation cost

\section{Carbon Permit Cost}

Carbon permit cost is the extra cost of emissions as a result of adding the new customer to the route. If the current vehicle assigned to route $r$ is of the type ICCV then carbon permit cost is estimated as:

carbon_permit $(i, p, k)= \begin{cases}+g h g \times \text { extra_energy_req }_{r}(i, p, k) & \text { if } k=I C C V \\ -g h g \times \text { Tot_energy }_{r} & \text { if } k=E C V\end{cases}$

where,

- $\quad g h g=$ amount of green house gas emission per liter of fuel

- Tot_energy $y_{r}=$ Total energy (fuel) required to traverse route $r$ before the insertion of customer $i$

If the current vehicle assigned to route $r$ is of the type ECV, then carbon permit cost is estimated as:

carbon_permit $(i, p, k)=\left\{\begin{array}{lr}+g h g \times \text { Tot }_{\text {energy }_{r}}(i, p) & \text { if } k=I C C V \\ 0 & \text { if } k=E C V\end{array}\right.$

where, 
- $\operatorname{Tot}_{\text {energy }}(i, p)=$ Total energy (fuel) required to traverse route $r$ after the insertion of customer $i$ at point $p$

\section{Extra Labor Cost}

Extra labor cost accounts for the increase in total operation time after the insertion of customer $i$ at insertion point $p$.

\section{$\underline{\text { Regret Score }}$}

Having estimated all the components of the penalty associated with the insertion of each un-routed customer $i$ to the existing routes in the partial solution, the regret score $\delta(i)$ is estimated as:

$\delta(i)=\operatorname{smin}_{r}\left(\rho_{r}(i)\right)-\min _{r}\left(\rho_{r}(i)\right)$

Where, $\operatorname{smin}_{r}$ denotes the second minimum value. In fact, the score $\delta(i)$ is the difference between the penalties of the second minimum cost insertion route and the first minimum cost insertion route. Having estimated the value of score $\delta(i)$ for all un-routed customers the one with the largest $\delta$ is selected and assigned to the minimum insertion cost route. Once a customer is assigned to a route $\mathrm{r}$, the $\delta$ scores are updated for the set of un-routed customers and it continues until all customers are routed. The pseudocode for the proposed constructive heuristic is given below.

input: Partial Solution

output: Initial Feasible Solution

for each un-routed customer $i$ do 
for each route $\mathrm{r}$ do compute the score $\rho_{r}(i)$;

Compute the score $\delta(i)$;

endfor

while the set of un-routed customers is not empty do

Identify the customer with the maximum value of $\delta(i)$;

Insert the customer $i$ in the best possible insertion point of the route $r$ with minimum $\rho_{r}(i)$

for each un-routed customer $i$ do

update the scores $\rho_{r}(i)$ and $\delta(i)$;

endfor

end while

\subsection{Improvement Heuristic Algorithm}

As mentioned before, the heuristic algorithm proposed in this dissertation is based on deconstruction of a large part of current solution and rebuilding it to generate better new solutions. Therefore, once a feasible initial solution is generated using the constructive heuristic, it should be destroyed based on a strategy and rebuilt using the same procedure explained in the constructive heuristic.

In order to destroy the current solution first a target route is identified. The target route is a route that can be served by a cheaper vehicle if a small set of customers is removed from it. In other words, if the total load served by route $r$ is $T L_{r}$ and it is served by vehicle type $h$, then the target route is a route minimizing excess load. Excess load is the nonnegative quantity of goods that cannot be delivered to the customers served by route $r$ using a cheaper vehicle of type $\mathrm{h}-1$ and is calculated using equation 5.9 
$E L_{r}=T L_{r}-Q_{h-1}$

where,

$$
\begin{aligned}
& E L_{r}=\text { Excess load of route } r \\
& T L_{r}=\text { Total load carried by vehicle serving route } r \\
& Q_{h-1}=\text { Loading Capacity of the cheaper vehicle of type } h-1
\end{aligned}
$$

Once a route is selected as a target route, it is stored in a Tabu list with infinite length such that it cannot be selected in any other iteration during the entire execution of the algorithm. Therefore, there might be a situation where we fail to find a target route. In this case, we prematurely terminate the algorithm and accept the current solution as the best solution found by the algorithm. Therefore, one of the stopping criteria in the improvement phase is failure to find a target route.

Given the target route $r$, each of its customers is considered as a target customer. The target customer is used to initialize the set of customers to be removed from the current solution. For each target customer in the target route, we find two routes with minimum insertion cost for the target customer and un-route all the customers in those two routes as well as the target customer. Then, the partial solution is transformed into a complete solution by using the regret algorithm described in the constructive heuristic part of this chapter. In fact, the un-routed customers are added to existing or new routes iteratively by using regret scores until no customer is left and the solution is complete. If the new solution is better than the previous solution, it is accepted as the new current solution and it is used in the next iteration of the improvement phase. On the other hand, 
if the new solution is not better than the previous solution, the previous solution remains as the best current solution for the next iteration of the improvement phase. This procedure continues until a time limit is reached or the algorithm fails to find a target route. The pseudocode for the improvement heuristic is given below.

Input: initial feasible solution to TDGVRP instance $\left[\ell_{0}, z_{0}\right]$

Output: $\left[\ell^{*}, \mathrm{z}^{*}\right]$

Initialization: $\ell^{*}=\ell_{0}, \mathrm{z}^{*}=z_{0}$

While not time limit do

$\ell=\ell^{*}$

Determine the non-tabu route $\bar{r}$ minimizing the excess load;

If no such route exists then stop;

Store $\bar{r}$ in tabu list with infinite length;

For each target customer $i$ in target route $\bar{r}$ do

Remove customer $i$ from route $\bar{r}$ of $\ell$;

Find the set of 2 routes with minimum insertion cost of $i$;

Remove all customers of the 2 routes from $\ell$;

Use the constructive heuristic to generate a new feasible solution $\mathrm{z}$

If $\mathrm{z}<\mathrm{z}^{*}$ then $\mathrm{z}^{*}=\mathrm{z}, \ell^{*}=\ell$

endfor

end while

Figure 5.4 to Figure 5.8 show the evolution of steps in the improvement phase for a network of 10 customers. In this network, there is one LEZ that includes three demand nodes and one charging station. The numbers in the boxes show the total load carried by 
the vehicles assigned to the routes and the optimal fleet size is listed in the table next to graphs.

Figure 5.4 is the initial solution found by the constructive heuristic. Based on this solution the optimal fleet size required to serve the customer demands in the network is one ICCV with the capacity of $4000 \mathrm{lbs}$, two ICCVs with the capacity of $6000 \mathrm{lbs}$, and one ECV with the capacity of $8000 \mathrm{lbs}$. The target route minimizing the excess load is shown in the red color in Figure 5.5. As mentioned before, once the target route is identified, each of its customers is considered as a target customer and for each target customer two routes with the minimum insertion cost are identified. In Figure 5.6, demand node $\mathrm{P}_{5}$ is chosen as a target customer and the two routes with minimum insertion cost for this node are shown in dotted lines. In Figure 5.7 all the customers in the two identified routes as well as the target customer are un-routed resulting in a partial solution. The partial solution is rebuilt by the constructive heuristic in Figure 5.8 and new routes are generated. As it can be seen in this figure the fleet size has changed to one ICCV with the capacity of $4000 \mathrm{lbs}$, and two ICCVs and one ECV with the battery capacity of $6000 \mathrm{lbs}$. In fact, the total fleet cost in the improvement phase is decreased by changing the fleet composition in a more efficient way. 




\begin{tabular}{|c|c|}
\hline $\begin{array}{c}\text { \# of } \\
\text { Required } \\
\text { Vehicles }\end{array}$ & $\begin{array}{c}\text { Load } \\
\text { Capacity }\end{array}$ \\
\hline $\mathbf{0}$ & 2000 \\
\hline $\mathbf{1}$ & 4000 \\
\hline $\mathbf{2}$ & 6000 \\
\hline $\mathbf{1}$ & 8000 \\
\hline $\mathbf{0}$ & 12000 \\
\hline
\end{tabular}

Figure 5. 4 Evolution of the Improvement Heuristic Algorithm - Part a

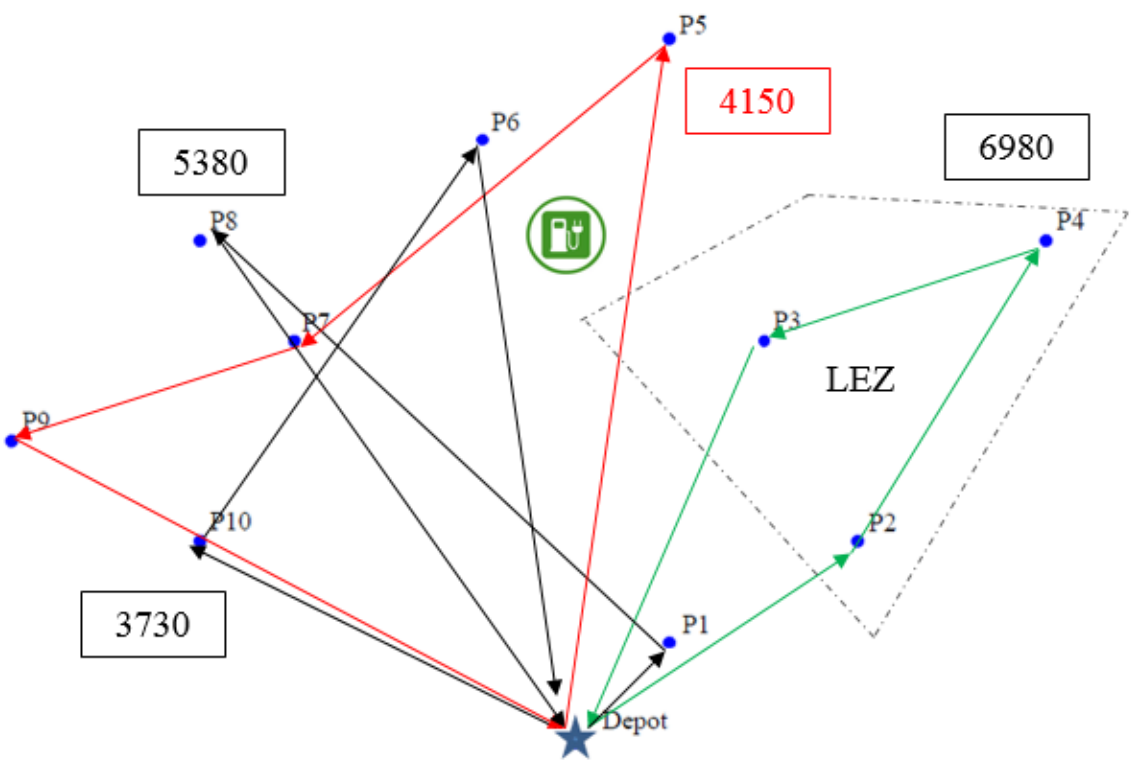

Figure 5. 5 Evolution of the Improvement Heuristic Algorithm - Part b 


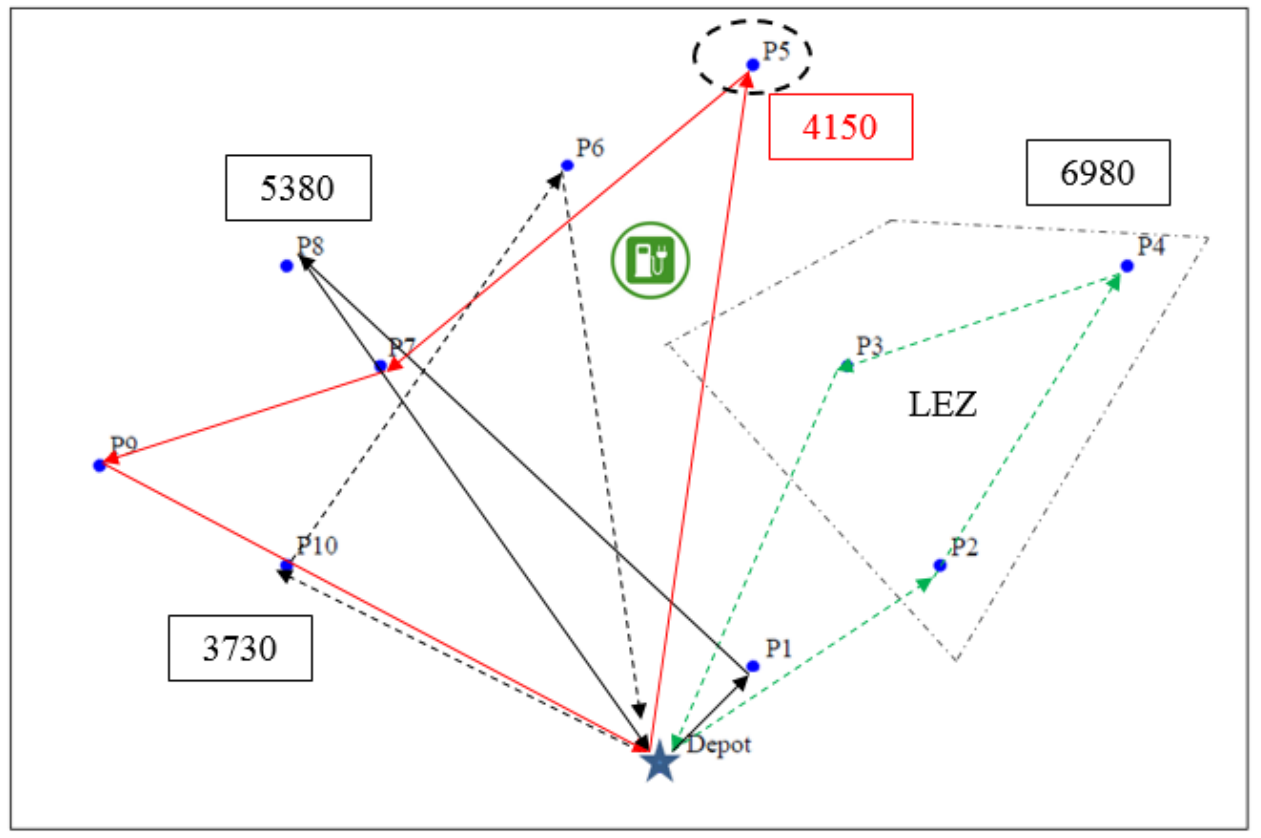

Figure 5. 6 Evolution of the Improvement Heuristic Algorithm - Part c

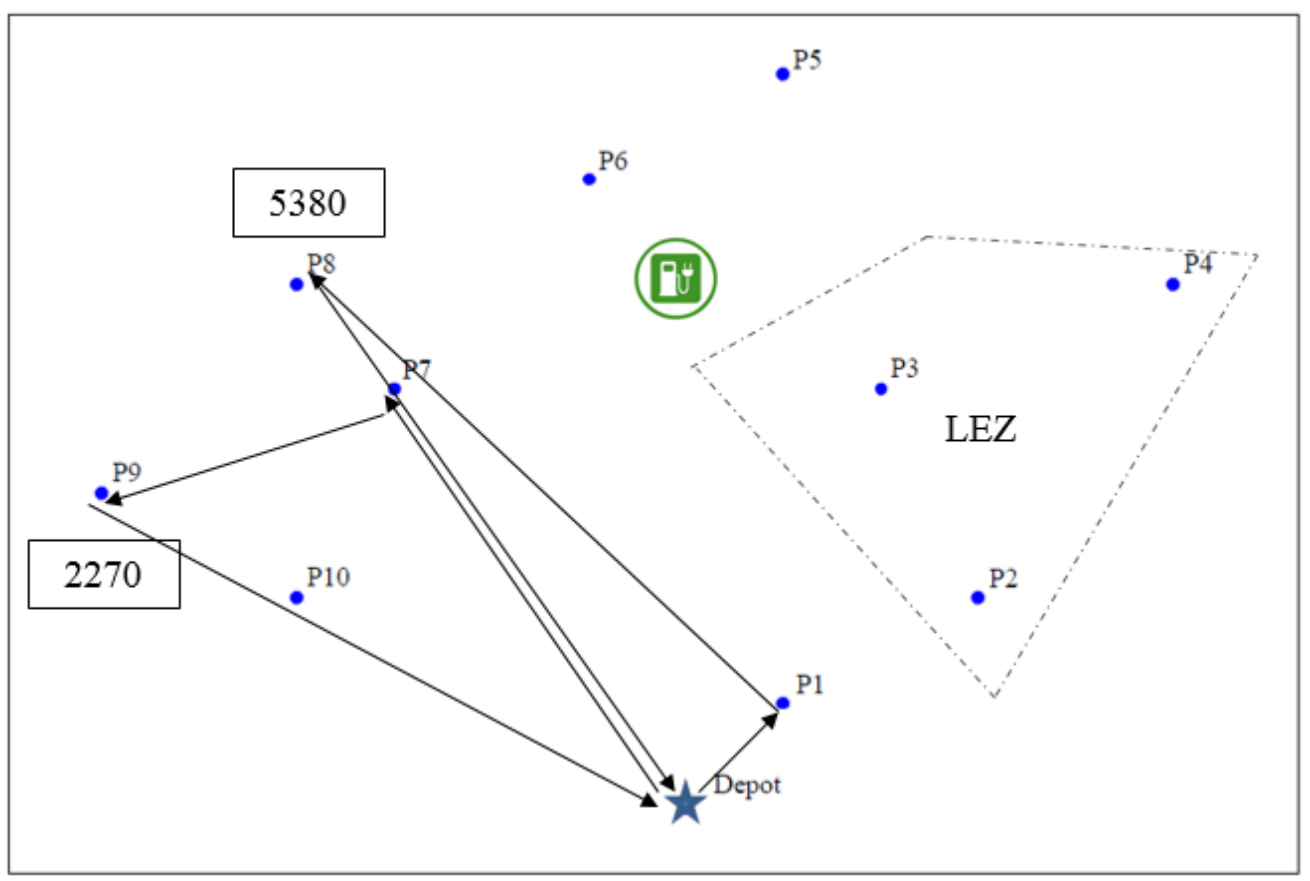

Figure 5. 7 Evolution of the Improvement Heuristic Algorithm - Part d 


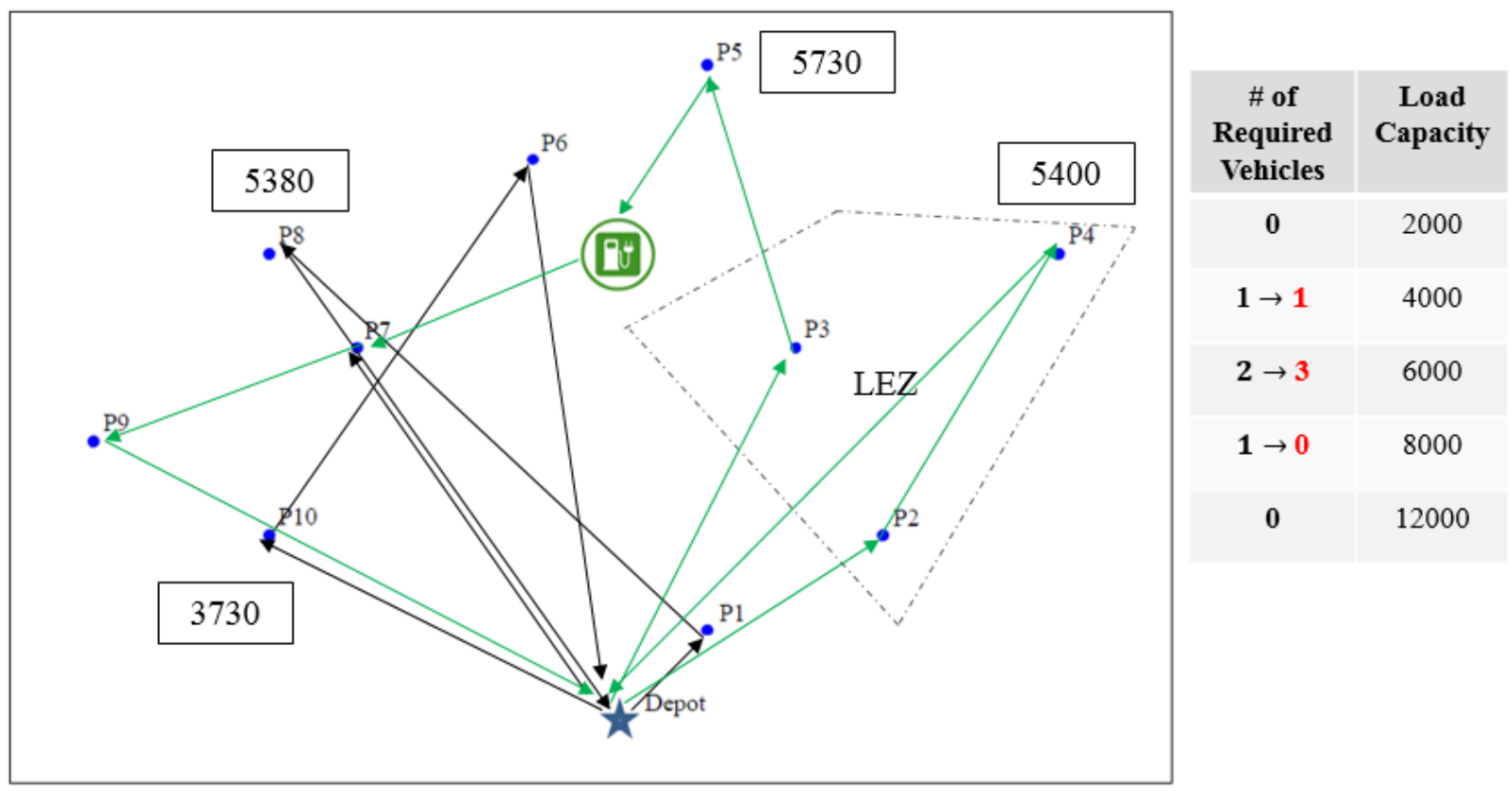

Figure 5. 8 Evolution of the Improvement Heuristic Algorithm - Part e

\subsection{Verification of The Heuristic Algorithm for Time-Dependent GVRP}

In order to verify the accuracy of the proposed heuristic algorithm in finding sound solutions, its performance is monitored by comparing the solutions generated by the algorithm with optimal solutions found by Xpress commercial solver for small size problems. For this purpose, a set of small size problems was defined. For each problem, a random network of demand nodes, charging stations, and depot were generated. The networks had different topology of customers, charging station and depot locations. For all the problem instances it was assumed that 2 types of electric trucks, with different loading and battery capacities, and 2 types of internal combustion engine trucks, with different loading capacities, were available. The planning period was divided into three time windows and a different travel time function was defined for each arc of the network 
during each of these time periods. Due to the fact that rush hour traffic might be different for opposite directions of a route, different travel time functions were defined for the two opposite directions of an arc in the network. Average acceleration rate on each direction of an arc in the network was randomly generated from a uniform distribution for each time period. The acceleration rates during morning and evening rush hours were randomly generated from the range $[0.3,0.5] \mathrm{m} / \mathrm{s}^{2}$. For the midday off-peak, the acceleration rate on arcs was randomly generated from the range $[0.1,0.3] \mathrm{m} / \mathrm{s}^{2}$. The customer demands were randomly generated from uniform distributions listed in Table 5.1. In all of the cases, it was assumed that all operated vehicles leave the depot at 8 am and all the ECVs are fully charged overnight. The characteristics of the generated small size problems are illustrated in Table 5.1. These characteristics are: number of customer nodes, number of charging stations, number of nodes in LEZ, LEZ penalty cost, demand distribution, carbon price and emission cap.

Each one of the defined problems was solved with both Xpress commercial solver and the proposed heuristic method and the results were compared. The heuristic running time, Xpress running time, and heuristic gap are shown for each problem in the Table 5.2. The numbers in black illustrate that Xpress could solve the problem optimally in less than one day. For example, in the case R1, the optimal solution to the problem is found in 3004 seconds by the Xpress solver and in 1.2 seconds by the proposed heuristic method and the gap between the solutions is $0.4 \%$. On the other hand, the numbers in red are associated to the cases where the Xpress solver was not able to find the optimal solution after 2 days. In these cases, the gaps report the difference between the heuristic solution and the lower 
bound found by the Xpress solver. As it can be seen in this table, the results show that the proposed heuristic works very well. While it might take more than 2 days for the commercial solver to find solutions to the small size problem, the proposed heuristic algorithm is capable of finding sound solutions with an average gap of $2.9 \%$ in a matter of seconds.

\subsection{Sensitivity Analysis on Heuristic Running Time}

As it was mentioned in section 5.3, the proposed heuristic algorithm in the improvement phase stops when either no target route can be found outside the Tabu list, or a predefined time limit is reached. In order to investigate the effect of the time limit on the quality of final solution, a sensitivity analysis was performed on running time and the improvement on the solutions was tracked. For this purpose, the algorithm was tested on randomly generated networks with 50 demand/customer nodes, and 3 charging station. In order to evaluate the average performance of the heuristic method, 10 random networks were generated with different characteristics. For each network, the location of customers, charging stations and depot were randomly generated as well as the customer demand and service time windows. LEZs of different sizes with different penalty costs were assumed. Moreover, different emission cap and carbon permit cost was considered for each network. 
Table 5. 1 Characteristics of Small Size Problems

\begin{tabular}{|c|c|c|c|c|c|}
\hline $\begin{array}{c}\text { Case } \\
\#\end{array}$ & $\begin{array}{c}\text { Number of } \\
\text { Demand } \\
\text { Nodes/Charging } \\
\text { Station }\end{array}$ & $\begin{array}{c}\text { Number of } \\
\text { nodes in LEZ/ } \\
\text { LEZ Cost }\end{array}$ & $\begin{array}{c}\text { Demand } \\
\text { Distribution } \\
(\text { Ibs })\end{array}$ & $\begin{array}{c}\text { Carbon } \\
\text { Price } \\
\left(\frac{\$}{\text { gram }}\right)\end{array}$ & $\begin{array}{c}\text { Emission } \\
\text { Cap } \\
\left(\frac{\text { gram }}{\text { day }}\right)\end{array}$ \\
\hline R1 & $5 / 1$ & $1 / 100$ & {$[1000,2500]$} & 0.5 & 30 \\
\hline R2 & $5 / 1$ & $1 / 100$ & {$[1500,2000]$} & 1 & 30 \\
\hline R3 & $5 / 1$ & $1 / 20$ & {$[500,1500]$} & 0.5 & 20 \\
\hline R4 & $5 / 1$ & $1 / 20$ & {$[1000,2500]$} & 1 & 20 \\
\hline R5 & $6 / 1$ & $2 / 20$ & {$[1000,2500]$} & 0.5 & 30 \\
\hline R6 & $6 / 1$ & $2 / 20$ & {$[1000,2500]$} & 1 & 30 \\
\hline R7 & $6 / 1$ & $2 / 100$ & {$[500,1500]$} & 2 & 20 \\
\hline R9 & $6 / 1$ & $2 / 20$ & {$[500,1500]$} & 1.5 & 20 \\
\hline
\end{tabular}


Table 5. 2 Comparison Between Xpress Solver and the Heuristic Method Solutions

\begin{tabular}{|c|c|c|c|c|c|}
\hline Case \# & $\begin{array}{l}\text { Number of } \\
\text { Constraints }\end{array}$ & $\begin{array}{l}\text { Number of } \\
\text { Variables }\end{array}$ & $\begin{array}{c}\text { Xpress } \\
\text { Running } \\
\text { Time }\end{array}$ & $\begin{array}{c}\text { Heuristic } \\
\text { Running } \\
\text { Time }\end{array}$ & $\begin{array}{c}\text { Solution } \\
\text { Gap }\end{array}$ \\
\hline $\mathrm{R} 1$ & 11844 & 8227 & $3004 \mathrm{sec}$ & $2.2 \mathrm{sec}$ & $0.4 \%$ \\
\hline $\mathrm{R} 2$ & 11844 & 8227 & $4108 \mathrm{sec}$ & $1.8 \mathrm{sec}$ & $0.1 \%$ \\
\hline R3 & 11844 & 8227 & $10723 \mathrm{sec}$ & $1.7 \mathrm{sec}$ & $2.8 \%$ \\
\hline $\mathrm{R} 4$ & 11844 & 8227 & $7283 \mathrm{sec}$ & $2.1 \mathrm{sec}$ & $1.5 \%$ \\
\hline R5 & 16577 & 10967 & $>2$ Day & $2.3 \mathrm{sec}$ & $7.6 \%$ \\
\hline R6 & 16577 & 10967 & $27220 \mathrm{sec}$ & $1.8 \mathrm{sec}$ & $2.2 \%$ \\
\hline R7 & 16577 & 10967 & $21720 \mathrm{sec}$ & $1.9 \mathrm{sec}$ & $0.3 \%$ \\
\hline R8 & 16577 & 10967 & $25274 \mathrm{sec}$ & $1.7 \mathrm{sec}$ & $1.8 \%$ \\
\hline R9 & 16577 & 10967 & $>2$ Day & $1.9 \mathrm{sec}$ & $9.8 \%$ \\
\hline R10 & 16577 & 10967 & $20836 \mathrm{sec}$ & $2.1 \mathrm{sec}$ & $2.3 \%$ \\
\hline
\end{tabular}

Figure 5.9 shows the improvement on initial solution found by the constructive heuristic as the running time of the improvement phase of heuristic increases. As it can be seen in this figure, the solution value changes with running time until it reaches a somehow 
saturation point. For the networks with 50 demand nodes the initial solution is found on average in 180 seconds, and no more improvements is achieved after running the improvement phase of the heuristic for more than 1000 seconds. It means that there is a threshold on improvements achieved by the heuristic and running the algorithm for more than this threshold would not result in better solutions. If the algorithm is not stopped by a time limit, on average it takes about 2600 seconds for the algorithm to stop due to not finding a target route to initialize solution deconstruction.

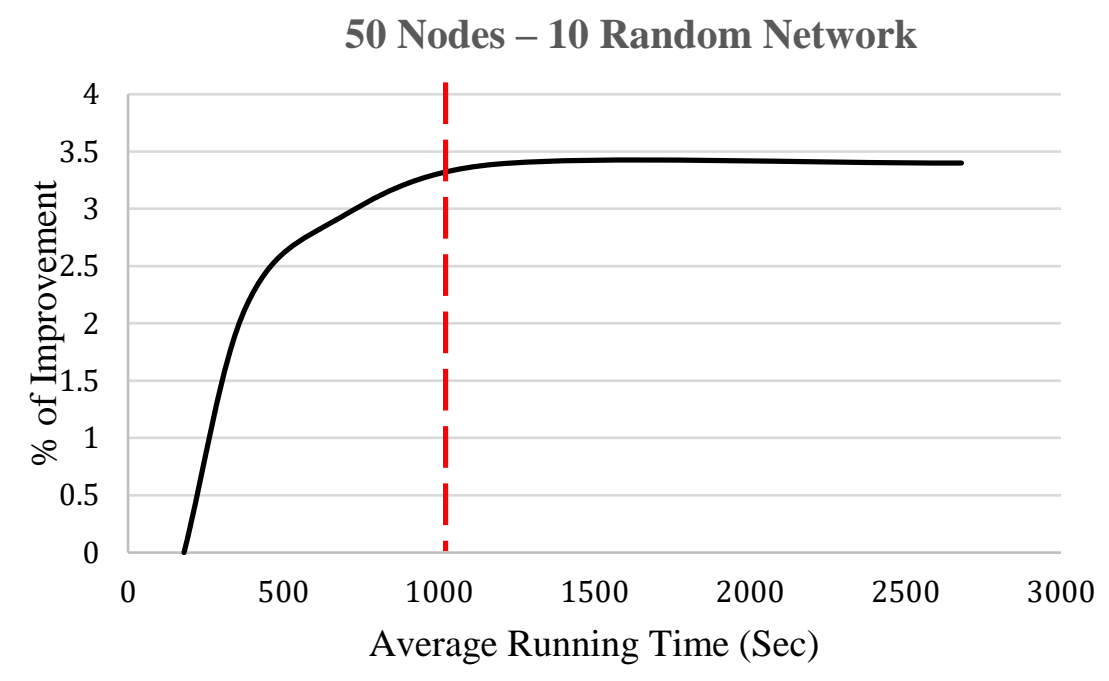

Figure 5. 9 Sensitivity Analysis of Heuristic Running Time on Solution Improvement

\subsection{Summary}

In this chapter first the proposed heuristic method was explained in detail. Then to see how the heuristic method performed, several cases were generated and the results of the heuristic method were compared to Xpress optimal solutions. The comparisons illustrated that the heuristic method is very promising and it can find very good solutions in a very 
short time. At the end of this chapter, a sensitivity analysis was performed on heuristic running time. It confirmed that the solution improves over time during the improvement phase of the heuristic method until it reaches a saturation point. It was seen that running the heuristic for any duration longer than this point does not result in any significant improvements on the final solution. 


\section{Chapter 6}

\section{CASE STUDY}

In this chapter, first the proposed heuristic algorithm is applied to a problem on a large size network to evaluate its performance on real world problems. Then a set of sensitivity analysis is performed on the parameters of the problem to investigate the heuristic's potential outcome in different situations. It is shown that the result of the sensitivity analysis could be used as a mean to evaluate the effectiveness of different policies such as Low Emission Zones and Emission Cap on the reduction of emission produced by delivery operations in an urban area.

\subsection{Case Study}

\subsubsection{Problem Characteristics}

Data on real world delivery operations such as FedEx or UPS was not available for this study. Therefore a network of 150 demand nodes was randomly generated in an area with the size of Washington DC, as shown in Figure 6.1. The generated network consisted of 150 demand nodes, one depot and five charging stations. It was assumed all vehicles depart from the depot at 8 in the morning and return back to depot at 6 in the afternoon. Therefore, three different travel time windows were considered for the operation: morning rush hour, midday off-peak, and evening rush hour. 


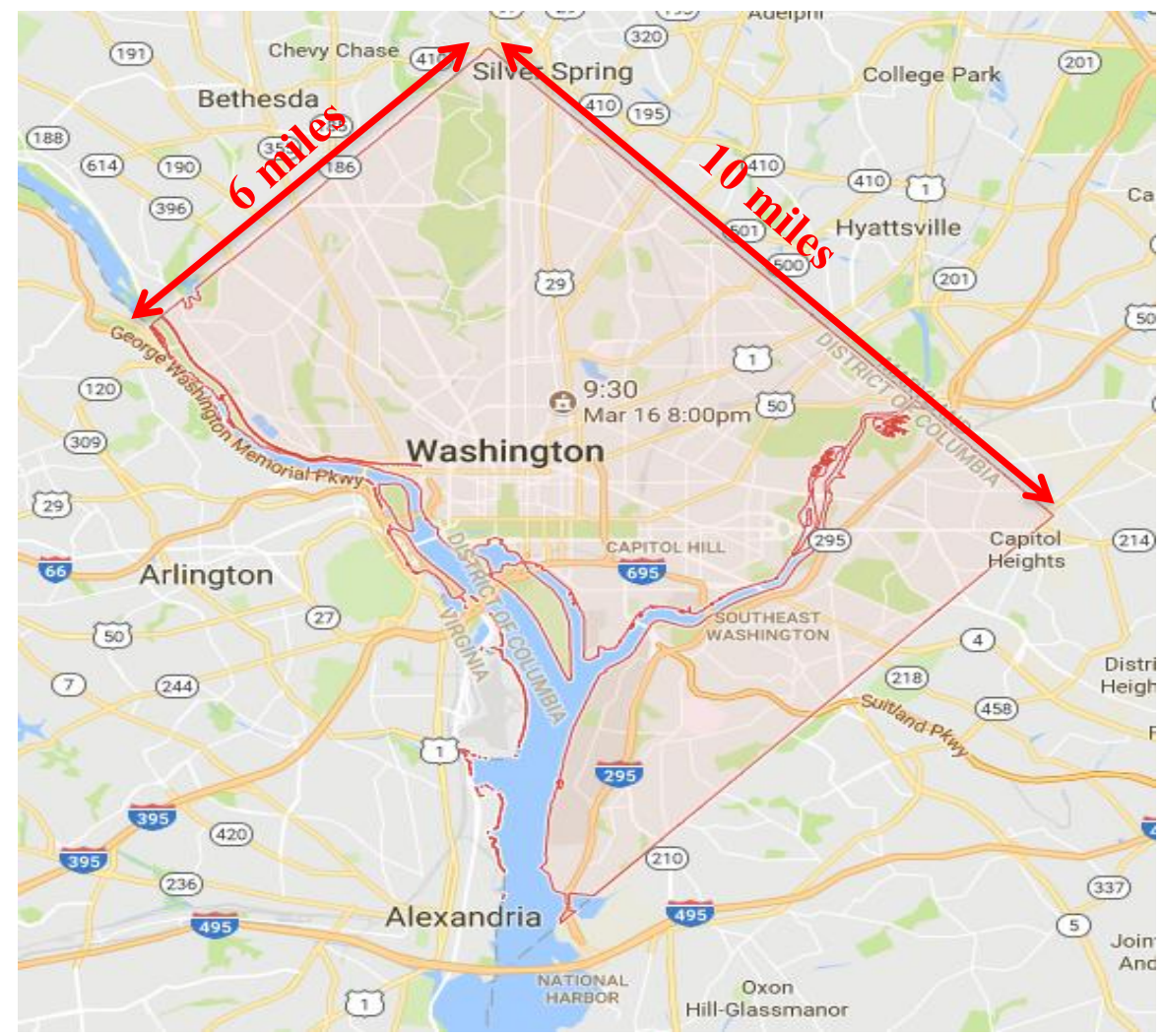

Figure 6. 1 Size of the Case Study

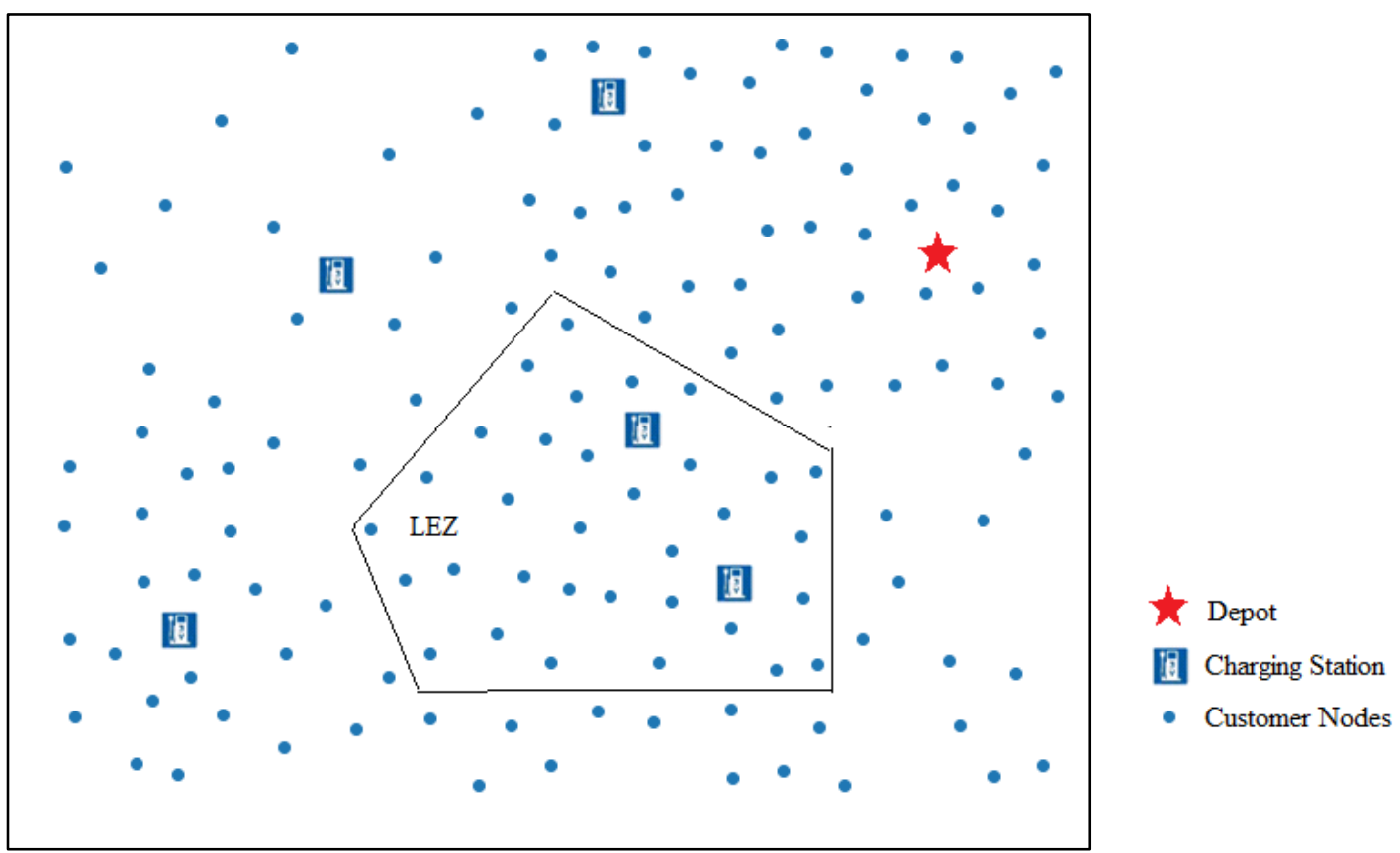

Figure 6. 2 Randomly Generated Network for Case Study 
In order to account for the variation of congestion level during the defined time windows, three different travel time functions were considered for each arc of the network along each direction. Moreover, an average acceleration rate was randomly generated for each direction of arcs in the network during each time window. The average speed and average acceleration rates were used to estimate the value of $(\alpha, \beta)$ and $\left(\alpha^{\prime}, \beta^{\prime}\right)$ required for estimation of the ICCV and ECV mechanical power requirement respectively. The quantity of demand and service time window for each customer in the network was randomly generated from uniform distributions.

A Time-Dependent GVRP was defined on the generated network. Table 6.1 summarizes the characteristics of the problem. A low emission zone with the penalty of $\$ 60$ for ICCVs was defined on the network. An emission cap of 200 grams/day was considered on the emissions produced by delivery operations. The price of carbon permit was set to $\$ 0.50 /$ gram, therefore any extra permit was purchased or sold at this price. The price of fuel and electricity was set to $\$ 0.76 /$ gram and $\$ 0.12 / \mathrm{kwh}$ respectively. The value of time was assumed to be $\$ 15 /$ hour. It was assumed three different types of ICCV and ECV are available with different loading and battery capacities. The characteristics of the vehicles are shown in Table 6.2. These characteristics are in terms of vehicle loading capacity, vehicle weight, and vehicle battery capacity where applicable. The vehicles are referred to by their sizes, Large, Medium and Small, in the next parts of this chapter. 
Table 6. 1 Characteristics of the Case Study

\begin{tabular}{|c|c|}
\hline Parameter & Value \\
\hline No. of Demand Nodes & 150 \\
\hline No. of Charging Stations & 5 \\
\hline No. of nodes in LEZ & $30(20 \%$ of total demand nodes $)$ \\
\hline LEZ Penalty (\$/day) & $\$ 60$ \\
\hline Emission Cap (gram/day) & 200 \\
\hline Carbon Permit Price (\$/gram) & 0.5 \\
\hline Electricity Cost $(\$ / \mathrm{kwh})$ & 0.12 \\
\hline Fuel Cost (\$/liter) & 0.76 \\
\hline Value of Time (\$/day) & 15 \\
\hline
\end{tabular}


Table 6. 2 Characteristics of the Available Vehicles

\begin{tabular}{|c|c|c|c|}
\cline { 2 - 4 } \multicolumn{1}{c|}{} & Small & Medium & Large \\
\hline Loading Capacity (LBs) & 5000 & 8000 & 12000 \\
\hline Vehicle Weight (LBs) & 2500 & 3500 & 5000 \\
\hline Battery Capacity for ECVs (kwh) & 4 & 5 & 6 \\
\hline
\end{tabular}

\subsubsection{Heuristic Solution}

The proposed heuristic method was used to find a solution to the defined problem. Figure 6.3 illustrates the improvement in the heuristic solution as the running time increases. The best solution is achieved by running the algorithm for 4300 seconds and no significant improvements is achieved by running the heuristic for longer durations. If not stopped at 4300 seconds the algorithm runs until there is no target route found which in this case is at 8000 seconds.

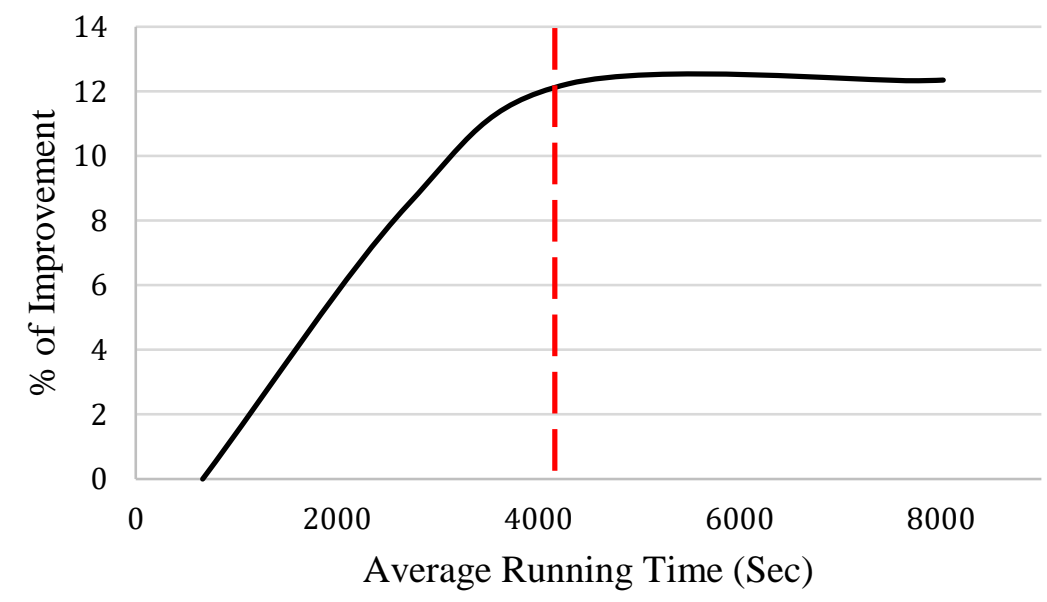

Figure 6. 3 Improvement in Heuristic Solution over Running Time 
The output of the heuristic method and the solution to the defined problem is given in Table 6.3. Based on the heuristic solution, the total operation cost for the defined problem is $\$ 2,747$, which includes both the vehicle acquisition cost and the routing cost. The best fleet size is found to be a mix of 10 large ICCVs, 3 large ECVs and 1 medium ECVs to serve the demand nodes in LEZ. The total emission produced is 307 grams. Due to the low cost of carbon permit, the emission cap is violated. In fact, the benefit in saving carbon permits does not offset the extra cost of ECVs comparing to ICCVs.

Table 6. 3 Solution to the Case Study

\begin{tabular}{|c|c|c|c|c|c|}
\hline $\begin{array}{c}\text { Total Cost } \\
(\mathbf{\$})\end{array}$ & No. of ICCV & No. of ECV & $\begin{array}{c}\text { Emission } \\
\text { (gram) }\end{array}$ & $\begin{array}{c}\text { Fuel } \\
\text { (liter) }\end{array}$ & $\begin{array}{c}\text { Electricity } \\
\text { (kwh) }\end{array}$ \\
\hline 2,747 & $10 \mathrm{~L}, 1 \mathrm{M}$ & $3 \mathrm{~L}$ & 317 & 119 & 25 \\
\hline
\end{tabular}

\section{$\underline{6.2 \text { Sensitivity Analysis }}$}

There are different parameters in the problem that can have significant effect on the solution output of heuristic. These parameters are:

- The extension of LEZ coverage area,

- LEZ Penalty Cost

- Emission cap,

- Emission Cost, and,

- Customer demand. 
The analysis of the solution sensitivity to these parameters provides a better vision of the heuristic's potential outcome in different situations. In this section, first the TimeDependent GVRP is solved for a basic scenario where there are no LEZ or emission cap regulations present. Then a set of sensitive analysis is performed for each of the specified parameters and the changes in solution is discussed.

\subsubsection{Base Scenario}

The base scenario is considered as a benchmark for sensitivity analysis. It means that the solution to the problem after changing the value of any of the parameters mentioned above are compared to the solution of the base scenario. The base scenario is defined on the network generated in section 6.1 and its characteristics is presented in Table 6.4. In the base scenario it is assumed that there is no LEZ zone or emission cap on the operations.

The solution of heuristic for the base scenario is presented in Table 6.5. As it can be seen in this figure when there is no LEZ present in the network and there is no limitation on emission produced by operations, the fleet is only composed of ICCVs. This is due to the less fixed costs and higher driving ranges of ICCVs compared to ECVs. Therefore, with the objective of minimizing total operation cost, ICCVs are preferred to ECVs in the base scenario. 
Table 6. 4 Characteristics of the Base Scenario

\begin{tabular}{|c|c|}
\hline Parameter & Value \\
\hline No. of Demand Nodes & 150 \\
\hline No. of Charging Stations & 5 \\
\hline No. of nodes in LEZ & None \\
\hline LEZ Penalty (\$/day) & None \\
\hline Emission Cap (gram/day) & None \\
\hline Carbon Permit Price (\$/gram) & None \\
\hline Electricity Cost & 0.12 \\
\hline Fuel Cost (\$/liter) & 0.76 \\
\hline Value of Time ( $\$ /$ day) & 15 \\
\hline
\end{tabular}

Table 6. 5 Solution to the Base Scenario

\begin{tabular}{|c|c|c|c|c|c|}
\hline $\begin{array}{c}\text { Total Cost } \\
\text { (\$) }\end{array}$ & No. of ICCV & No. of ECV & $\begin{array}{c}\text { Emission } \\
\text { (gram) }\end{array}$ & $\begin{array}{c}\text { Fuel } \\
\text { (liter) }\end{array}$ & $\begin{array}{c}\text { Electricity } \\
\text { (kwh) }\end{array}$ \\
\hline 2444 & $13 \mathrm{~L}, 1 \mathrm{~S}$ & 0 & 440 & 156 & 0 \\
\hline
\end{tabular}




\subsubsection{LEZ Coverage}

It is expected that as the coverage of LEZ increases and more demand nodes fall in the LEZ zone, more number of ICCVs be replaced by ECVs. This is true if the LEZ penalty cost exceeds the difference in the employment cost of the two different types of vehicle. Otherwise if the LEZ penalty plus the ICCV employment cost is still less that the ECV employment cost, no change is expected in the fleet of base scenario. In order to evaluate the effect of LEZ coverage on the solution of the heuristic, five different cases with different levels of LEZ coverage are solved by the proposed heuristic method. Each of the cases is defined on the network of base scenario, with 150 demand nodes and 5 charging stations. However problem characteristics are different in each case. The characteristics of the defined problems is given in Table 6.6. In all of the cases there is no emission cap on the operations meaning that there is no limitation on the total amount of emission produced by ICCVs serving customer demand. The only limitation in the use of ICCVs is the LEZ with a daily penalty of $\$ 100$. The price of electricity, fuel, and value of time is the same as the base scenario.

The solution of the heuristic to the defined problems is presented in Table 6.7. As it can be seen, if the LEZ penalty cost is more than the difference in employment cost of the two types of vehicle, as the coverage of LEZ increases, more number of ICCVs are replaced by ECVs and, as a result, the total emission produced by vehicles while routing is reduced. Therefore, increasing the coverage of LEZ is in favor of the ultimate goal, which is encouraging the use of ECVs and reducing the produced emission as a result. However, this change in the fleet mix increases the total operation cost. Figure 6.4 
illustrates the change in operation cost versus the change in total emission as the LEZ coverage increases. As it is shown in the figure, the minimum LEZ coverage of $10 \%$ reduces the emission by $13 \%$ while increase the cost of operation by only $4 \%$. Obviously, the maximum reduction in emission is achieved when all demand nodes are located in a LEZ. Replacing all ICCVs with ECVs result in almost $37 \%$ increase in the operation cost.

Table 6. 6 Characteristics of the Cases for LEZ Coverage Sensitivity Analysis

\begin{tabular}{|c|c|c|c|c|c|}
\cline { 2 - 6 } \multicolumn{1}{c|}{} & \multicolumn{5}{c|}{ Value } \\
\hline Parameter & Case 1 & Case 2 & Case 3 & Case 4 & Case 5 \\
\hline No. of Demand Nodes & 150 & 150 & 150 & 150 & 150 \\
\hline No. of Charging Stations & 5 & 5 & 5 & 5 & 5 \\
\hline LEZ Coverage & $10 \%$ & $20 \%$ & $30 \%$ & $60 \%$ & $100 \%$ \\
\hline LEZ Penalty (\$/day) & 100 & 100 & 100 & 100 & 100 \\
\hline Emission Cap (gram/day) & None & None & None & None & None \\
\hline Carbon Permit Price (\$/gram) & None & None & None & None & None \\
\hline Electricity Cost & 0.12 & 0.12 & 0.12 & 0.12 & 0.12 \\
\hline Fuel Cost (\$/liter) & 0.76 & 0.76 & 0.76 & 0.76 & 0.76 \\
\hline Value of Time (\$/day) & 15 & 15 & 15 & 15 & 15 \\
\hline
\end{tabular}


Table 6. 7 LEZ Coverage Sensitivity Analysis Result

\begin{tabular}{|c|c|c|c|c|c|c|}
\cline { 2 - 7 } \multicolumn{1}{c|}{} & $\begin{array}{c}\text { Base } \\
\text { Scenario }\end{array}$ & Case 1 & Case 2 & Case 3 & Case 4 & Case 5 \\
\hline Total Cost (\$) & 2444 & 2553 & 2754 & 3002 & 3019 & 3342 \\
\hline \# of ICCV & $13 \mathrm{~L}, 1 \mathrm{~S}$ & $11 \mathrm{~L}, \mathrm{M}$ & $7 \mathrm{~L}, 2 \mathrm{M}$ & $4 \mathrm{~L}, 2 \mathrm{M}$ & $4 \mathrm{~L}, 1 \mathrm{M}$ & 0 \\
\hline \# of ECV & 0 & $1 \mathrm{~L} 1 \mathrm{M}$ & $5 \mathrm{~L}$ & $8 \mathrm{~L}$ & $8 \mathrm{~L} \mathrm{1M}$ & $12 \mathrm{~L} 2 \mathrm{M}$ \\
\hline Emission (gram) & 440 & 381 & 233 & 175 & 120 & 0 \\
\hline Fuel (liter) & 156 & 142 & 87 & 65 & 47 & 0 \\
\hline Electricity (kwh) & 0 & 15 & 53 & 91 & 91 & 120 \\
\hline
\end{tabular}

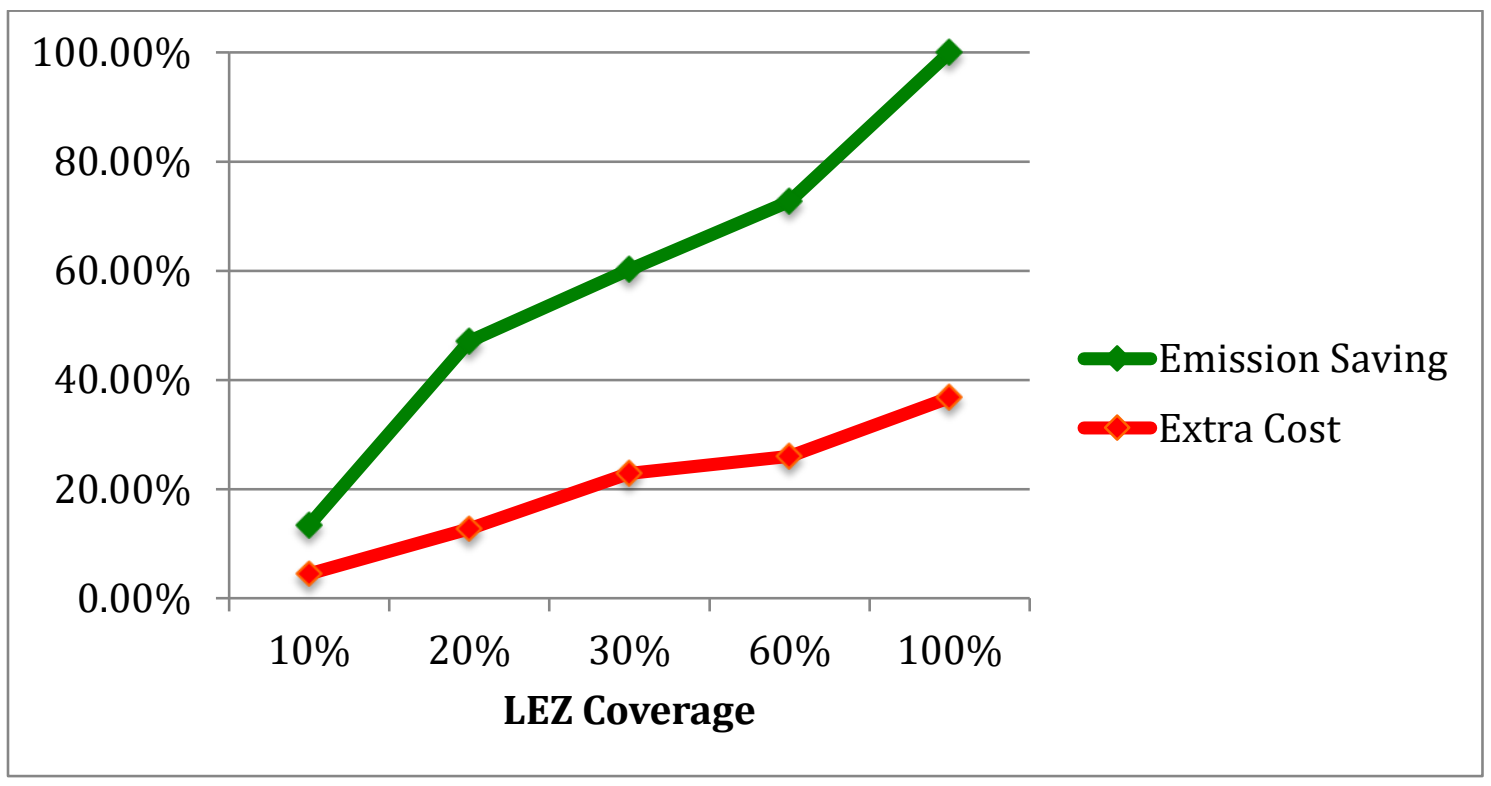

Figure 6. 48 Emission Saving and Extra Cost over Different LEZ Coverage

\subsubsection{LEZ Penalty Cost}

Another factor that affects the fleet mix and route of the vehicles is the LEZ penalty cost. If the operation cost of ICCV plus the LEZ penalty cost is less that the operation cost of 
$\mathrm{ECV}$, then an electric truck replaces the conventional truck. Therefore, it is expected to have different fleet mix and routing plans with different values of penalty for a same level of LEZ coverage. In order to verify the performance of the heuristic for different values of the LEZ penalty cost, four different scenarios were considered as shown in Table 6.8. It was assumed that there is an LEZ with coverage of 30\% in the network. There was no emission cap considered for the routing operation and the value of time, fuel, and electricity was assumed to be the same as the base scenario.

The result of the heuristic is shown in Table 6.9. When there is a LEZ with the penalty cost of $\$ 30$ (case 1), the fleet mix is changed from 13 large and 1 small ICCVs, to 12 large and 2 medium ICCVs. This change in the fleet size increases the operation cost and slightly reduces the routing emissions.

However, when the penalty cost increases from $\$ 30$ to $\$ 50$ some of the ICCVs are replaced with ECVs to avoid paying the LEZ penalty cost. The change in the fleet reduces the emission significantly while resulting in an increase in the total operation cost. This change in the fleet size continues when the LEZ penalty cost increases to $\$ 80$ but it stops afterwards. It means that if the LEZ penalty cost is increased for any value greater than $\$ 80$, no changes would be expected in the fleet size and routing plan of vehicles. The change in emission and total operation cost is illustrated in Figure 6.5. 
Table 6. 8 Characteristics of the Cases for LEZ Penalty Cost Sensitivity Analysis

\begin{tabular}{|c|c|c|c|c|}
\cline { 2 - 5 } \multicolumn{1}{c|}{} & \multicolumn{4}{c|}{ Value } \\
\hline Parameter & Case 1 & Case 2 & Case 3 & Case 4 \\
\hline No. of Demand Nodes & 150 & 150 & 150 & 150 \\
\hline No. of Charging Stations & 5 & 5 & 5 & 5 \\
\hline LEZ Coverage & $30 \%$ & $30 \%$ & $30 \%$ & $30 \%$ \\
\hline LEZ Penalty (\$/day) & 30 & 50 & 80 & 100 \\
\hline Emission Cap (gram/day) & None & None & None & None \\
\hline Carbon Permit Price (\$/gram) & None & None & None & None \\
\hline Electricity Cost (\$/kwh) & 0.12 & 0.12 & 0.12 & 0.12 \\
\hline Fuel Cost (\$/liter) & 0.76 & 0.76 & 0.76 & 0.76 \\
\hline Value of Time (\$/day) & 15 & 15 & 15 & 15 \\
\hline
\end{tabular}

\subsubsection{Emission Price and Emission Cap}

Another factor affecting the fleet size as well as the routing plan of trucks is the emission cap and trade policy. From the solution to the base scenario, it is observed that if there is no emission cap on the delivery operations, all the customers are served by ICCVs and the total amount of emission produced is about 440 grams. However if there is a cap on the amount of emission produced during the operation and extra emissions are penalized, there might be a change in fleet size and routing plans. Extend of this change depends on the 
penalty charged for extra emission known as carbon permit cost. In this section, a sensitivity analysis is performed on the solution of the heuristic for different carbon permit pierces under different emission caps. Table 6.10 shows the characteristics of the cases defined for the sensitivity analysis. It is assumed that there is no LEZ in the network and the only regulation limiting the use of ICCVs is the cap and trade policy. For each level of emission cap five different values of carbon permit cost are tested starting from $\$ 1 /$ gram to $\$ 5 /$ gram. The cost of fuel, electricity and value of time are assumed to be the same as the base scenario.

Table 6. 9 LEZ Penalty Cost Sensitivity Analysis Result

\begin{tabular}{|c|c|c|c|c|c|}
\cline { 2 - 6 } \multicolumn{1}{c|}{} & $\begin{array}{c}\text { Base } \\
\text { Scenario }\end{array}$ & Case 1 & Case 2 & Case 3 & Case 4 \\
\hline Total Cost (\$) & 2,444 & 2,819 & 3,280 & 3,501 & 3,501 \\
\hline \# of ICCV & $13 \mathrm{~L}, 1 \mathrm{~S}$ & $12 \mathrm{~L}, 2 \mathrm{M}$ & $7 \mathrm{~L}, 2 \mathrm{M}$ & $4 \mathrm{~L}, 2 \mathrm{M}$ & $4 \mathrm{~L} 2 \mathrm{M}$ \\
\hline \# of ECV & 0 & 0 & $5 \mathrm{~L}$ & $8 \mathrm{~L}$ & $8 \mathrm{~L}$ \\
\hline Emission (gram) & 440 & 416 & 275 & 175 & 175 \\
\hline Fuel (liter) & 156 & 147 & 102 & 65 & 65 \\
\hline Electricity (kwh) & 0 & 0 & 53 & 91 & 91 \\
\hline
\end{tabular}




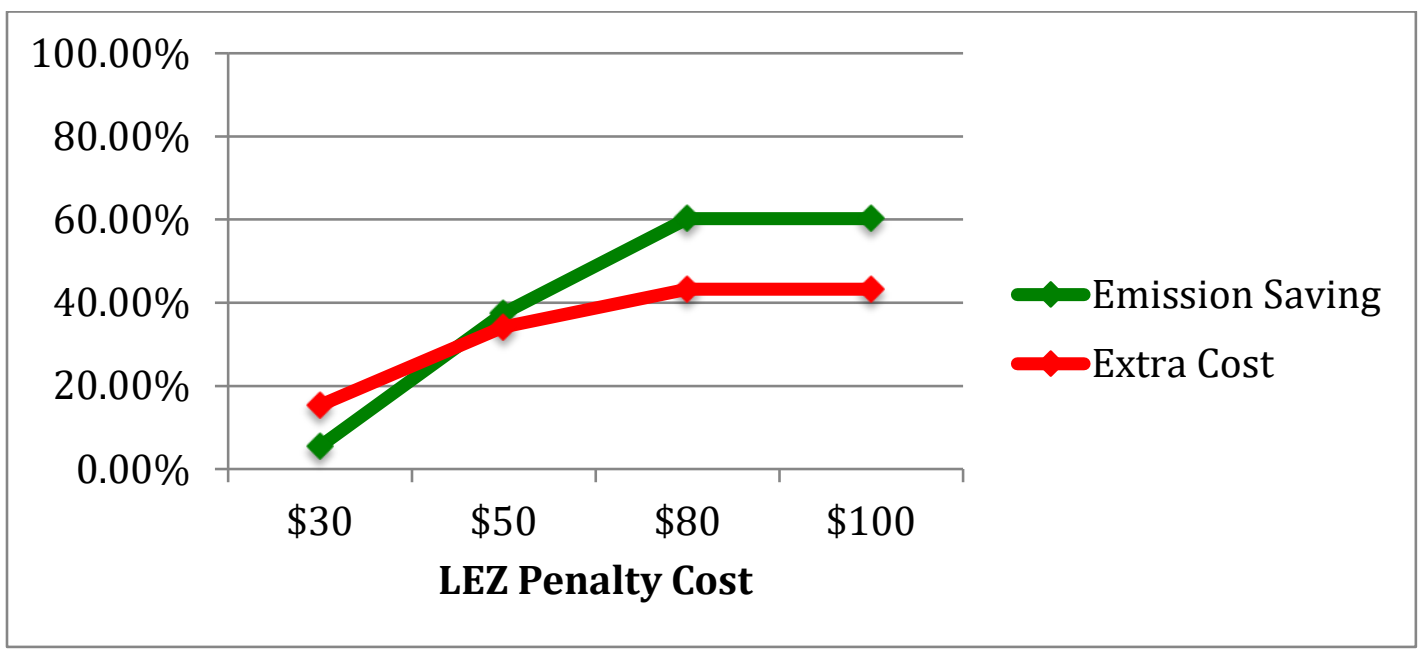

Figure 6. 5 Emission Saving and Extra Cost over Different LEZ Penalty Cost

The solution found by heuristic for each case is presented in Table 6.11 for the emission cap of 200 grams and in the Table 6.12 for emission cap 300 grams respectively. As it is shown, as the price of carbon increases from $1 \$$ to $5 \$$ more ICCVs are replaced with ECVs until the fleet becomes pure ECVs. While there is an overall increasing trend in emission savings over different carbon prices, the change in total operation cost does not follow a constant trend. It first increases and then starts to decrease. The decrease in total cost happens when the benefits earned by selling extra carbon permits outweighs the extra employment cost of ECVs. It should be noted that the solutions to the cases are the same for the both emission caps in terms of fleet mix and routing cost but the total operation cost is different. It is expected due to the different levels of benefit achieved by selling extra carbon permit cost. The changes in total operation cost and emission savings for different carbon prices are illustrated in Figure 6.6 and Figure 6.7. 
Table 6. 10 Characteristics of the Cases for Emission Price Sensitivity Analysis

\begin{tabular}{|c|c|c|c|c|c|}
\cline { 2 - 6 } \multicolumn{1}{c|}{} & \multicolumn{5}{c|}{ Value } \\
\hline Parameter & Case 1 & Case 2 & Case 3 & Case 4 & Case 5 \\
\hline No. of Demand Nodes & 150 & 150 & 150 & 150 & 150 \\
\hline No. of Charging Stations & 5 & 5 & 5 & 5 & 5 \\
\hline LEZ Coverage & None & None & None & None & None \\
\hline LEZ Penalty (\$/day) & None & None & None & None & None \\
\hline Emission Cap (gram/day) & $\{300$, & $\{300$, & $\{300$, & $\{300$, & $\{300$, \\
& $200\}$ & $200\}$ & $200\}$ & $200\}$ & $200\}$ \\
\hline Carbon Permit Price (\$/gram) & 1 & 2 & 3 & 4 & 5 \\
\hline Electricity Cost (\$/kwh) & 0.12 & 0.12 & 0.12 & 0.12 & 0.12 \\
\hline Fuel Cost (\$/liter) & 0.76 & 0.76 & 0.76 & 0.76 & 0.76 \\
\hline Value of Time (\$/day) & 15 & 15 & 15 & 15 & 15 \\
\hline
\end{tabular}

\subsubsection{Customer Demand}

In the problem defined in this dissertation, demand at customer locations is average demand. A set of sensitivity analysis is performed to investigate the change in the fleet size if demand at each customer location is more than its average demand. For this purpose, 
the heuristic solution was used to solve the problem defined in the case study (section 6.1) for three different scenario of:

- $5 \%$ increase in demand

- $10 \%$ increase in demand

- $20 \%$ increase in demand

Table 6. 11 Emission Cost Sensitivity Analysis Result - Emission Cap $=200$

\begin{tabular}{|c|c|c|c|c|c|c|}
\cline { 2 - 7 } \multicolumn{1}{c|}{} & $\begin{array}{c}\text { Base } \\
\text { Scenario }\end{array}$ & Case 1 & Case 2 & Case 3 & Case 4 & Case 5 \\
\hline Total Cost (\$) & 2,444 & 2,573 & 2,762 & 2,704 & 2,550 & 2,395 \\
\hline \# of ICCV & $13 \mathrm{~L}, 1 \mathrm{~S}$ & $12 \mathrm{~L}, 2 \mathrm{M}$ & $7 \mathrm{~L}, 2 \mathrm{M}$ & $2 \mathrm{M}$ & $1 \mathrm{M}$ & 0 \\
\hline \# of ECV & 0 & 0 & $5 \mathrm{~L}$ & $12 \mathrm{~L}$ & $12 \mathrm{~L}, 1 \mathrm{M}$ & $13 \mathrm{~L}, 1 \mathrm{M}$ \\
\hline Emission (gram) & 440 & 377 & 203 & 26 & 12 & 0 \\
\hline Fuel (liter) & 156 & 140 & 76 & 10 & 4 & 0 \\
\hline Electricity (kwh) & 0 & 0 & 65 & 110 & 129 & 132 \\
\hline
\end{tabular}

Table 6.13 shows the result of the heuristic for the different scenarios. In all of the scenarios there is a change in fleet mix and size over different demands. For example, if the demand at each customer location is $5 \%$ more the fleet mix is changed from $(10 \mathrm{~L}, 1$ M) ICCV, and (3L) ECV to (10 L, $1 \mathrm{~S}) \mathrm{ICCV}$ and (3L 1M) ECV. Therefore, as it is expected, the fleet size and mix changes with variations in demand. If the number of fleets for each vehicle type is known and prefixed, still the developed mathematical model can be used in order to find the optimal route for vehicles. 
Table 6. 12 Emission Cost Sensitivity Analysis Result - Emission Cap = 300

\begin{tabular}{|c|c|c|c|c|c|c|}
\cline { 2 - 7 } \multicolumn{1}{c|}{} & $\begin{array}{c}\text { Base } \\
\text { Scenario }\end{array}$ & Case 1 & Case 2 & Case 3 & Case 4 & Case 5 \\
\hline Total Cost (\$) & 2444 & 2473 & 2562 & 2404 & 2150 & 1895 \\
\hline \# of ICCV & $13 \mathrm{~L}, 1 \mathrm{~S}$ & $12 \mathrm{~L}, 2 \mathrm{M}$ & $7 \mathrm{~L}, 2 \mathrm{M}$ & $2 \mathrm{M}$ & $1 \mathrm{M}$ & 0 \\
\hline \# of ECV & 0 & 0 & $5 \mathrm{~L}$ & $12 \mathrm{~L}$ & $12 \mathrm{~L}, 1 \mathrm{M}$ & $13 \mathrm{~L}, 1 \mathrm{M}$ \\
\hline Emission (gram) & 440 & 377 & 203 & 26 & 12 & 0 \\
\hline Fuel (liter) & 156 & 140 & 76 & 10 & 4 & 0 \\
\hline Electricity (kwh) & 0 & 0 & 65 & 110 & 129 & 132 \\
\hline
\end{tabular}

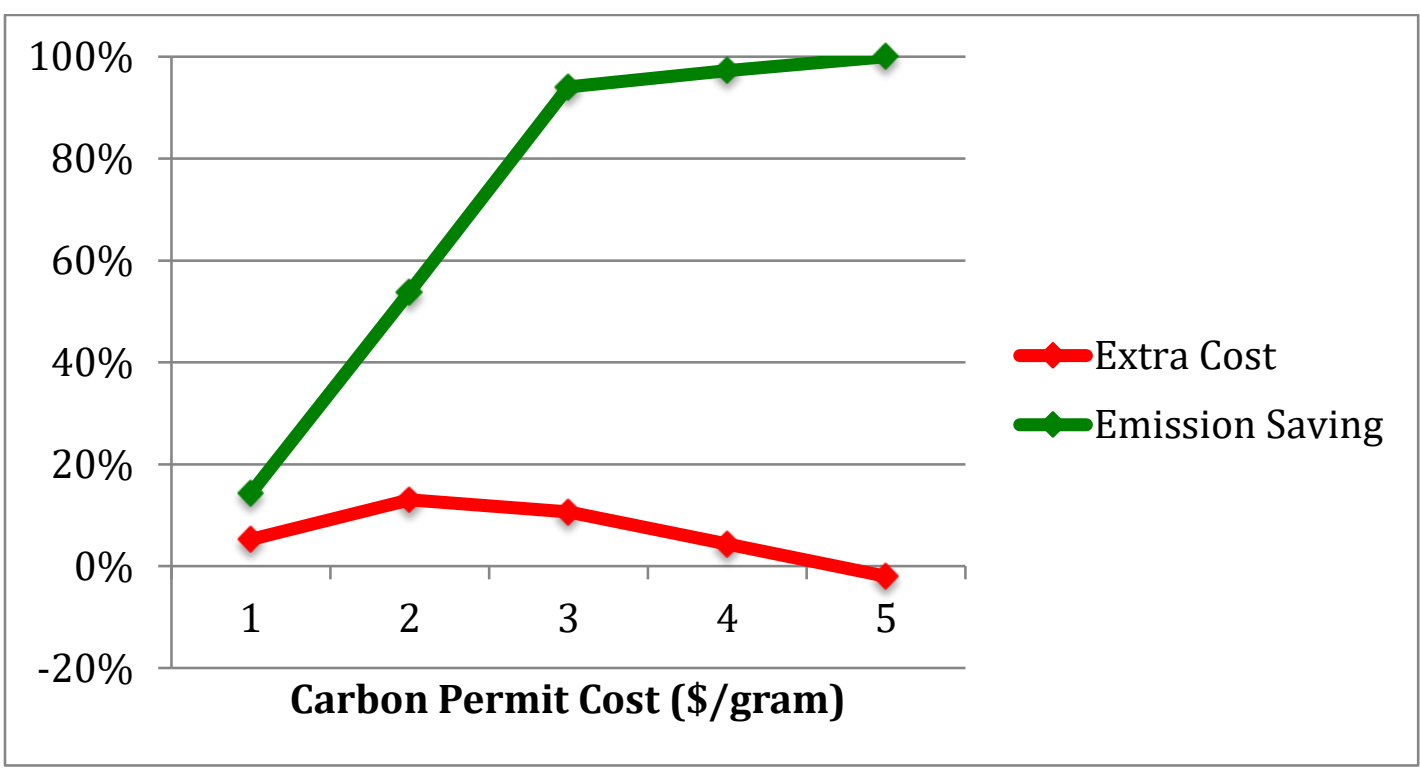

Figure 6. 6 Emission Saving and Extra Cost over Different Emission Cost - Emission Cap=200 


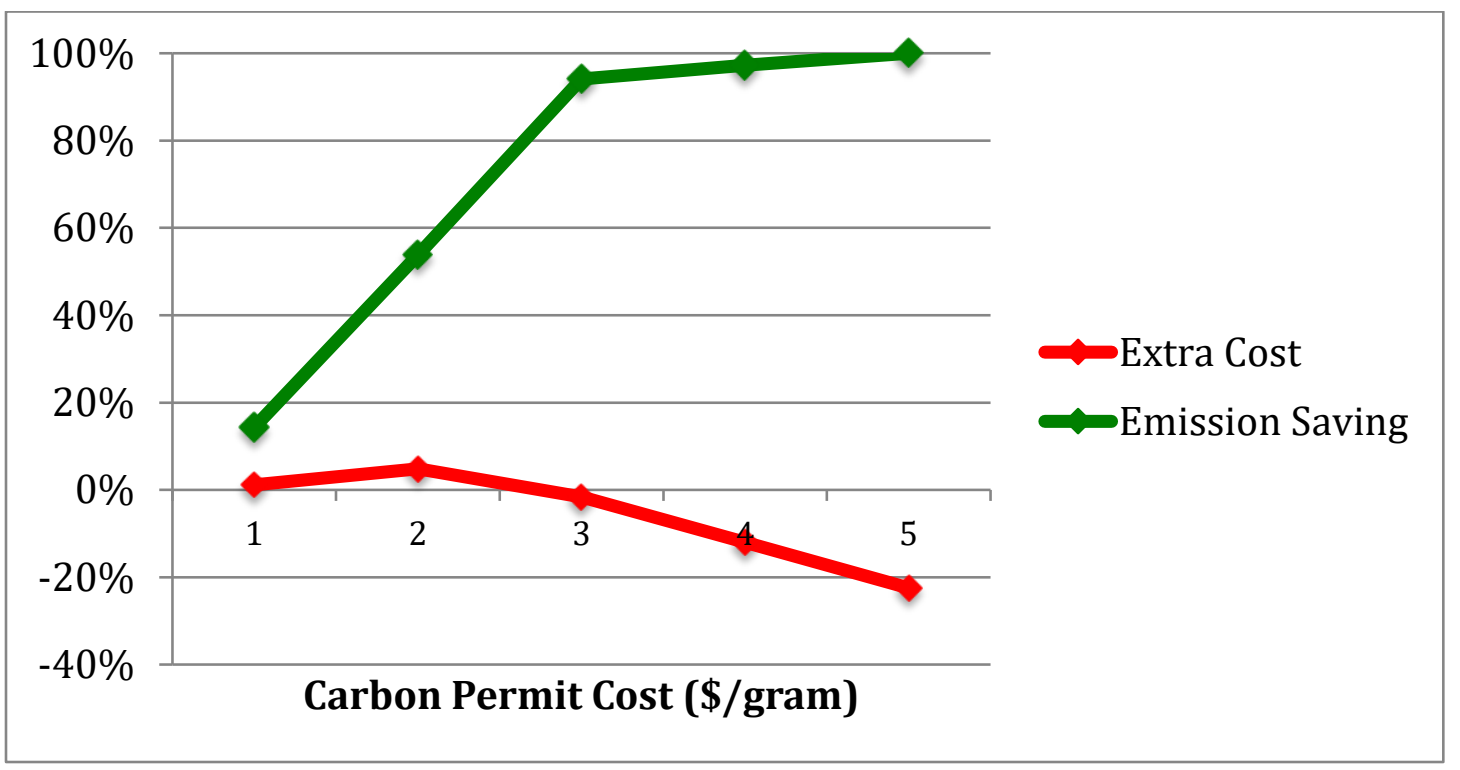

Figure 6. 7 Emission Saving and Extra Cost over Different Emission Cost - Emission Cap=300

Table 6. 13 Result of Sensitivity Analysis on Demand

\begin{tabular}{|c|c|c|c|}
\hline Case \# & Demand & \# of ICCV & \# of ECV \\
\hline Base Case Study & No change & $10 \mathrm{~L} \mathrm{1M}$ & $3 \mathrm{~L}$ \\
\hline 1 & $+5 \%$ & $10 \mathrm{~L} \mathrm{1S}$ & $3 \mathrm{~L} \mathrm{1M}$ \\
\hline 2 & $+10 \%$ & $11 \mathrm{~L}$ & $4 \mathrm{~L}$ \\
\hline 3 & $+20 \%$ & $13 \mathrm{~L}$ & $3 \mathrm{~L} \mathrm{1S}$ \\
\hline
\end{tabular}

\subsection{Summary}

The proposed heuristic method was used to solve the Time-Dependent GVRP on a large size network. The improvement of solution over different running time of the heuristic method was monitored and an improvement of maximum $12 \%$ in final solution was seen in the improvement phase of heuristic method. The solution to the large size case study 
proved the efficiency of the heuristic algorithm in considering the tradeoffs between the employment of ICCVs and ECVs due to different policies. Moreover, a set of sensitivity analysis was performed on the parameters of the defined Time-Dependent GVRP. The parameters included the LEZ coverage, LEZ penalty cost, emission price and emission cap, as well as customer demand. The result of sensitivity analysis showed that the low emission zone or emission cap \& trade policies might encourage the replacement of ICCVs with ECVs for logistics operations which is favorable due to the significant reductions in emission. On the other hand, these policies might increase the total cost of company operations. In fact, it was shown that the developed model in this dissertation can be used by companies to evaluate the effect of the green logistic policies such as LEZ zone and emission cap on the last mile delivery operations in terms of the changes in fixed cost of fleet acquisition as well as the variable operation cost. 


\section{Chapter 7}

\section{SUMMARY, CONCLUSIONS, AND RECOMMENDATIONS FOR FUTURE RESEARCH}

\subsection{Summary and Conclusions}

In this research a special case of GVRP with a mixed fleet of heterogeneous electric and internal combustion engine commercial vehicles was studied. Two different formulations were proposed to solve two different variants of the problem. The first formulation was developed for a green vehicle routing problem where travel time on arcs is constant over the whole operation period. Then, the developed formula was modified and extended to account for the variations in travel time during different time periods of a day so that different levels of congestion could be taken into account while routing vehicles to serve customer demands. Through the developed formulations, the optimal fleet size and the minimum cost routing plan were found. The cost included:

- The vehicle fixed cost,

- The routing cost in the form of electric energy requirement or fuel consumption, labor cost, customer inconvenience costs that result from breaking service time windows, Low Emission Zone penalty cost and carbon permit cost.

While previous studies in the field of GVRP with mixed fleet of electric and conventional vehicles, focus only on the limitations of electric vehicles such as high 
purchase cost and limited driving range, the variant of GVRP studied in this dissertation tries to account for the limitations of both vehicle types. These limitations are in the form of Low Emission Zone penalty cost and emission cap for internal combustion engine vehicles and high purchase cost and limited driving range for electric vehicles. Low Emission Zone and Emission Cap are the policies used by government in some countries to encourage the use of green vehicles by imposing some limitation on the use of conventional vehicles. Therefore, the solution to the Time-Dependent GVRP defined in this study finds the best fleet size and routing plan to serve customers by taking into account the tradeoffs between the two types of vehicle.

Moreover, the GVRP defined in this study takes into account the variations in travel time. In fact the best routing plan is identified through consideration of different levels of congestion on arcs in the network during different time of a day. Therefore, the vehicle energy requirements are estimated more accurately, which is even more important in the case of eclectic vehicle due to the high sensitivity of electric vehicle's driving range to the energy consumption rate.

In order to account for the variations in travel time, continuous travel time functions were used. In fact, the planning period was divided into a number of time windows and travel time was formulated as a function of departure time in each of these time windows. The use of continuous travel time functions instead of step functions assured the conservancy of FIFO concept and provided a more realistic presentation of travel time variations. In fact, by the use of continuous travel time functions any kind of travel time variation was accounted for. 
As it is well known, Time-Dependent VRP is an NP-hard problem and there is a need for a heuristic solution algorithm to solve the problem for real size large networks. In this study a heuristic algorithm was proposed based on the Ruin and Recreate (RR) approach, a new class of algorithms introduced by Schrimpf, et al. (2000). A constructive heuristic was defined to generate initial feasible solution to the problem. The initial solution was further improved by deconstructing a large part of it and then, rebuilding it with the constructive heuristic. This algorithm was preferred over the local search algorithms as it is expected to provide better solutions due to the diversification effect embedded in it by deconstructing a large part of the solution.

To verify the performance of the proposed heuristic method, the results from the heuristic was compared to the solutions found by Xpress commercial solver. A set of small size problems with less than 8 demand nodes was generated. For some of the problems Xpress was able to find the exact solutions. If the exact solution was not available after running the Xpress for 2 days the solver was stopped and the heuristic solution was compared with the lower bound found by Xpress. The comparison between the Xpress solution and the heuristic solution showed that the proposed heuristic method is capable of producing good results within a very short time.

In order to verify the performance of the heuristic on real world large size problems, the proposed heuristic method was used to solve a case study defined on a randomly generated network with the size of Washington DC. Moreover, in order to see how the heuristic method is performing when the parameters in the problem are changing, an extensive sensitivity analysis was performed in this research. The parameters considered 
for sensitivity analysis on the case study and the results obtained from the analysis are as follows:

- LEZ Coverage: It was shown that as the coverage of LEZ increases more number of ICCVs are replaced by ECVs. While the cost of operation increases, there is a significant reduction in the emission. A low emission zone, which covers $20 \%$ of the demand, was seen to increase the cost of operation by $12 \%$, and reduce the emission by almost half.

- LEZ Penalty Cost: The result of sensitivity analysis on LEZ penalty cost showed that as the LEZ penalty cost exceeds a threshold ICCVs start to be replaced with ECVs. After some point a saturation point is achieved and increasing the LEZ penalty cost does not lead to any more changes in the fleet size and operation cost.

- Emission Price and Emission Cap: The heuristic solution for different values of carbon permit cost showed that as the cost of carbon increases more number of ICVVs are replaced by ECVs due to the extra benefits from emission savings. Moreover, it was seen that, for a same value of carbon permit, as the daily limit on emission increases, the emission savings do not change but the operation cost decreases due to the saving from selling carbon permit.

- Customer Demand: The solution of heuristic for the case study was compared to the solutions for different higher levels of demand. It was seen that as the demand increases the fleet size changes. However, in the studied scenarios it was assumed that the demand at all locations has the same amount of increase, which might not be the case in real world situations. 
The overall conclusions of this research can be outlined as followed.

- The proposed formulation for both GVRP and Time-Dependent GVRP can handle tradeoffs between the limitation on both types of vehicle, as well as variations in travel time during different periods of operation.

- The proposed heuristic method performs well and provides fairly good results for the generated test problems in terms of solution accuracy and run time, when compared with the exact solution for small problems.

- The result of the sensitivity analysis performed on the solution of heuristic with respect to parameters of LEZ coverage, LEZ penalty cost, emission cap and carbon price, and the customer demand shows the effectiveness of the heuristic algorithm in considering the tradeoffs between ICCVs and ECVs under different LEZ and cap and trade policies.

\subsection{Recommendations for Future Research}

There are several interesting avenues for future research. In this section some of these recommendations are discussed.

\section{- Lower Bound}

In this research the heuristic solutions were compared with the Xpress optimal solutions. This comparison showed good results and the errors were acceptable in the examples compared. However the comparison was done only on the examples that Xpress could handle. Xpress cannot solve large size problems even in a very long 
running time, so the comparison of heuristic method with optimal solutions is not done on the real size problems. The systematic approach to see how the heuristic method performs is to find a good lower bound for this problem that is missing in this research and is highly recommended for future studies.

\section{- Multi Depot}

The problem studied in this dissertation is defined on a network with a single depot. However, in real world operations, there might be more than one depot serving demand in the network. Therefore, in future, the developed model could be further improved by accounting for multiple depots.

\section{- Energy Consumption Model}

The energy consumption model used in this dissertation is a very comprehensive model that takes into account the effect of vehicle load, speed, and acceleration rate as well as road altitude on the vehicle energy requirements. Although, road altitude is accounted for in this model, it fails to estimate the amount of the energy that is regenerated by electric vehicles while on downhill. Therefore, developing a model that takes into account the energy regenerated by electric vehicles on downhill could improve the results on routing and charging station visit by electric vehicles.

\section{- Sensitivity Analysis on Battery Recharging Time}

In this dissertation, charging stations were assumed to be of battery swapping type with constant service time for all vehicles regardless of the level of the battery available upon visit to a charging station. Although, battery swapping stations are currently in practice and might be the future of charging stations, sensitivity analysis 
can be performed on the charging time to investigate how the variations in the amount of time to recharge the battery would affect the total cost, fleet size and vehicle routes.

\section{- Effect of Green Logistics Policies on Society's Welfare}

The model developed in this dissertation was focused on minimizing the total operation cost of a company while accounting for limitations in the adoption of ECVs and ICCVs as a result of government green logistics policies. The output of the model was used to evaluate the changes in the operation cost of company and the total emission produced as a result of changes in the fleet mix and routings. In future, it would be interesting to study the effect of these policies from a global perspective in order to evaluate the effects of green logistics policies on society's welfare. 


\section{References}

1. Alwakiel, H.N. (2011). Leveraging Weigh-In-Motion (WIM) data to estimate linkbased heavy-vehicle emissions. PhD thesis. Portland State University, USA.

2. Ángel Felipe, M. Teresa Ortuño, Giovanni Righini, \& Gregorio Tirado (2014). A heuristic approach for the green vehicle routing problem with multiple technologies and partial recharges, Transportation Research Part E: Logistics and Transportation Review, 71, 111-128.

3. Ardekani, S., Hauer, E., \& Jamei, B. (1996). Traffic impact models. In Traffic flow theory (pp. 1-7). Washington, DC, USA.

4. AustriaTech. (2014). Electric fleets in urban logistics: Improving urban freight efficiency in small and medium-sized historic towns. Published as part of the ENCLOSE project, funded by Intelligent Energy Europe (IEE), Vienna, Austria. URL

5. Baldacci, R., Mingozzi, A., \& Calvo, R. W. (2011). An exact method for the capacitated location-routing problem. Operations Research, 59, 1284-1296.

6. Balseiro, S.R., Loiseau, I., Ramonet, J. (2011). An Ant Colony algorithm hybridized with insertion heuristics for the Time Dependent Vehicle Routing Problem with Time Windows. Computers \& Operations Research, 38(6), 954-966.

7. Barco, J., A., Guerra, L. Muoz, \& N. Quijano (2012). Optimal Routing and Scheduling of charge for electric vehicles: Case study. Universidad de los andes, Bogota, Colombia. URL http://arxiv.org/ftp/arxiv/papers/1310/1310.0145.pdf. (Accessed November 2015)

8. Bard, J., Huang, L., Dror, M., \& Jaillet, P. (1998). A branch and cut algorithm for the VRP with satellite facilities. IIE Transactions 30, 821-834.

9. Barth, M., \& Boriboonsomsin, K. (2008). Real-world CO2 impacts of traffic congestion. Transportation Research Record: Journal of the Transportation Research Board, 2058(1), 163-171.

10. Barth, M., Younglove, T., \& Scora, G. (2005). Development of a heavy-duty diesel modal emissions and fuel consumption model. Technical report. UC Berkeley: California Partners for Advanced Transit and Highways (PATH), California, USA. http://www.path.berkeley.edu/PATH/Publications/PDF/PRR/2005/PRR-200501\%.pdf (Accessed November 2015).

11. Bektas, T., \& Laporte, G. (2011). The Pollution-Routing Problem, Transportation Research Part B: Methodological, 45(8), 1232-1250.

12. Bektas, T., \& Laporte, G. (2011). The pollution-routing problem. Transportation Research Part B: Methodological, 45(8), 1232-1250.

13. Bertsimas, D. J. (1992). A vehicle routing problem with stochastic demand. Operations Research, 40, 574-585.

14. Bigazzi, A. Y., \& Bertini, R. L. (2009). Adding green performance metrics to a transportation data archive. Transportation Research Record: Journal of the Transportation Research Board, 2121(1), 30-40. 
15. Brian A. Davis, \& Miguel A. Figliozzi (2013). A methodology to evaluate the competitiveness of electric delivery trucks, Transportation Research Part E: Logistics and Transportation Review, 49(1) 8-23.

16. Chang C. Business models and public policies for EV charging stations in China; 2010.

URL http://www.changce.org/attachments/559_evstation_businessmodel_changce_20 101018.pdf. (Accessed October 2015)

17. Chen, H.-K., Hsueh, C.-F., \& Chang, M.-S. (2006). The real-time time-dependent vehicle routing problem. Transportation Research Part E: Logistics and Transportation Review, 42(5), 383-408.

18. Chen, Z.-L., \& Xu, H. (2006). Dynamic column generation for dynamic vehicle routing with time windows. Transportation Science, 40, 74-88.

19. Cheung, B. K. S., Choy, K. L., Li, C.-L., Shi, W., \& Tang, J. (2008). Dynamic routing model and solution methods for fleet management with mobile technologies. International Journal of Production Economics, 113, 694-705.

20. Clarke, G., \& Wright, J. W. (1964). Scheduling of vehicles from a central depot to a number of delivery points. Operations Research, 12, 568-581.

21. Cook, T. M., \& Russell, R. A. (1978). A simulation and statistical analysis of stochastic vehicle routing with time constraints. Decision Sciences, 9, 673-687.

22. Christofides, N., A. Mingozzi, P. Toth. (1979). The vehicle routing problem. N. Christofides, A. Mingozzi, P. Toth, C. Sandi, eds. Combinatorial Optimization. John Wiley \& Sons, Chichester, UK, 315-338.

23. Dantzig, G., Ramser, R. (1959). The truck dispatching problem. Management Science 6, 80-91.

24. Daniel, S. E., Diakoulaki, D. C., \& Pappis, C. P. (1997). Operations research and environmental planning. European Journal of Operational Research, 102, 248-263.

25. Demir, E., Bektas, T., \& Laporte, G. (2011). A comparative analysis of several vehicle emission models for road freight transportation. Transportation Research Part D: Transport and Environment, 6(5), 347-357.

26. Demir, E., Bektas, T., \& Laporte, G. (2012). An adaptive large neighborhood search heuristic for the pollution-routing problem. European Journal of Operational Research, 223(2), 346-359.

27. Demir, E., Bektas, T., \& Laporte, G. (2013). The bi-objective pollution-routing problem. European Journal of Operational Research, 232(3), 464-478.

28. Den Boer E., S. Aarnink, F. Kleiner, J. Pagenkopf. (2013). Zero emission trucks: An overview of state-of-the-art technologies and their potential. CE Delf and DLR, commissioned by the International Council on Clean Transportation (ICCT). URL http://www.cedelft.eu/publicatie/zero_emission_trucks/1399. (Accessed September 2015)

29. Desaulniers, G., Errico, F., Irnich, S., \& Schneider, M. (2014). Exact algorithms for electric vehicle-routing problems with time windows. Les Cahiers du GERAD G-2014-110, GERAD, Montréal, Canada. 
30. Dominik Goeke, \& Michael Schneider (2015). Routing a mixed fleet of electric and conventional vehicles, European Journal of Operational Research, 245(1),8199.

31. Dondo, R., \& Cerdá, J. (2007). A cluster-based optimization approach for the multi-depot heterogeneous fleet vehicle routing problem with time windows. European Journal of Operational Research, 176, 1478-1507.

32. Dror, M., Laporte, G., \& Louveaux, F. V. (1993). Vehicle routing with stochastic demands and restricted failures. ZOR-Methods and Models of Operations Research, 37, 273-283.

33. Eglese, R. W., \& Black, D. (2010). Optimizing the routing of vehicles. In A. McKinnon, S. Cullinane, M. Browne, \& W. Whiteing, (Eds.), Green Logistics: Improving the environmental sustainability of logistics (pp. 215-228). Great Britain/KoganPage.

34. Electrification Coalition. 2013. State of the plug-in electric vehicle market. URL http://electrificationcoalition.org/sites/default/files/EC_State_of_PEV_Market_Fi nal_1.pdf. (Accessed September 2015).

35. European Union Emission Standards. Low Emission Zones. URL https://www.dieselnet.com/standards/eu/lez.php (Accessed October 2015).

36. F.G. Gheysens, B.L. Golden, \& A.A. Assad (1983). A relaxation heuristic for the fleet size and mix vehicle routing problem. In Proceedings of SE AIDS Meeting, Williamsburg, Virginia.

37. Figliozzi, M. A. (2012). The time dependent vehicle routing problem with time windows: benchmark problems, an efficient solution algorithm, and solution characteristics. Transportation Research Part E: Logistics and Transportation Review, 48(3), 616-636.

38. G. Hiermann, J. Puchinger, and R. F. Hartl (2014). The electric eet size and mix vehicle routing problem with time windows and recharging stations. Working Paper.

URL http://prolog.univie.ac.at/research/publications/downloads/Hie_2014_638.pdf. (Accessed November 2015)

39. Gendreau, M., Guertin, F., Potvin, J.-Y., \& Séguin, R. (2006). Neighborhood search heuristics for a dynamic vehicle routing problem with pick-ups and deliveries. Transport Research Part C: Emerging Technologies, 14, 157-174.

40. Gendreau, M., Guertin, F., Potvin, J.-Y., \& Séguin, R. (2006). Neighborhood search heuristics for a dynamic vehicle routing problem with pick-ups and deliveries. Transport Research Part C: Emerging Technologies, 14, 157-174.

41. Gendreau, M., Laporte, G., \& Séguin, R. (1996). Stochastic vehicle routing. European Journal of Operational Research, 88, 3-12.

42. Giosa, I., Tansini, I., \& Viera, I. (2002). New assignment algorithms for the multidepot vehicle routing problem. Journal of the Operational Research Society, 53, 977-984.

43. Golden, B. L., \& Stewart, W., Jr. (1978). Vehicle routing with probabilistic demands. In D. Hogben \& D. Fife (Vol. Eds.), Computer science and statistics: 
tenth annual symposium on the interface, NBS special publication 503. National Bureau of Standards, Washington, DC.

44. Haghani A., \& Jung S. (2005). A dynamic vehicle routing problem with timedependent travel times, Computers \& Operations Research, 32(11) 2959-2986.

45. Hong, L. (2012). An improved LNS algorithm for real-time vehicle routing problem with time windows. Computers \& Operations Research, 39, 151-163.

46. http://ntl.bts.gov/lib/32000/32700/32779/DOT_Climate_Change_Report__April_2010_-_Volume_1_and_2.pdf. (Accessed September 2015).

47. http://www.austriatech.at/files/get/834747f18fdc9538376c9314a4d7652/austriate ch_electricfleets_broschuere.pdf. (Accessed September 2015)

48. IPCC (1995). IPCC 2nd assessment report: climate change 1995. Geneva: IPCC.

49. IPCC (2007). IPCC 2nd assessment report: climate change (2007). Geneva: IPCC.

50. Jaillet, P., \& Odoni, A. R. (1988). The probablistic vehicle routing problem. In B. L. Golden \& A. A. Assad (Eds.), Vehicle routing: methods and studies. Amsterdam:North-Holland.

51. Jaillet, P., \& Odoni, A. R. (1988). The probablistic vehicle routing problem. In B. L. Golden \& A. A. Assad (Eds.), Vehicle routing: methods and studies. Amsterdam: North-Holland.

52. Jézéquel, A. (1985). Probabilistic vehicle routing problem. M.Sc. Dissertation, Department of Civil Engineering, Massachusetts Institute of Technology, Cambridge, MA.

53. Jovicic' , N. M., Boškovic' , G. B., Vujic' , G. V., Jovic ‘ic' , G. R., Despotovic' , M. Z., Milovanovic' , D. M., et al. (2010). Route optimization to increase energy efficiency and reduce fuel consumption of communal vehicles. Thermal Science, 14, 67-78.

54. Kara, I., Kara, B. Y., \& Yetis, M.K. (2007). Energy minimizing vehicle routing problem. In A. Dress, Y. Xu, \& B. Zhu, (Eds.), Combinatorial optimization and applications, Lecture notes in computer science Vol. 4616. Berlin/Heidelberg.

55. Kim L. Namsan electric bus cruises into international lime-light (2011). URL http://www.cnngo.com/seoul/life/namsan-electric-bus-cruises-limelight-262862

(Accessed October 2015)

56. Kok, A. L., Hans, E. W., \& Schutten, J. M. J. (2012). Vehicle routing under timedependent travel times: the impact of congestion avoidance. Computers \& Operations Research, 39(5), 910-918.

57. Kritzinger, S., Doerner, K. F., Hartl, R. F., Kiechle, G., Stadler, H., \& Manohar, S. S. (2012). Using traffic information for time-dependent vehicle routing. Procedia -Social and Behavioral Sciences, 39, 217-229.

58. Kuo, Y. (2010). Using simulated annealing to minimize fuel consumption for the time-dependent vehicle routing problem. Computers \& Industrial Engineering, 59(1), 157-165.

59. Kuo, Y. (2010). Using simulated annealing to minimize fuel consumption for the time-dependent vehicle routing problem. Computers \& Industrial Engineering, 59(1), 157-165. 
60. Lambert, V., Laporte, G., \& Louveaux, F. V. (1993). Designing collection routes through bank branches. Computers \& Operations Research, 20, 783-791.

61. Laporte, G., Louveaux, F. V., \& Mercure, H. (1992). The vehicle routing problem with stochastic travel times. Transportation Science, 26, 161-170.

62. Laporte, G., Nobert, Y., \& Desrochers, M. (1985). Optimal routing under capacity and distance restrictions. Operations Research 33 (5), 1050-1073.

63. Lecluyse, C., Sörensen, K., \& Peremans, H. (2013). A network-consistent timedependent travel time layer for routing optimization problems. European Journal of Operational Research, 226(3), 395-413.

64. Li, X., Tian, P., \& Leung, C. H. (2010). Vehicle routing problems with time windows and stochastic travel and service times: models and algorithm. International Journal of Production Economics, 125, 137-145.

65. Maden, W., Eglese, R. W., \& Black, D. (2010). Vehicle routing and scheduling with time-varying data: A case study. Journal of the Operational Research Society, 61(3), 515-522.

66. Maden, W., Eglese, R., \& Black, D. (2010). Vehicle routing and scheduling with time varying data: a case study. Journal of the Operational Research Society, 61, 515-522.

67. Madsen, O. B. G., Tosti, K., \& Vælds, J. (1995). A heuristic method for dispatching repair men. Annals of Operations Research, 61, 213-226.

68. Malandraki, C., \& Daskin, M. S. (1992). Time dependent vehicle routing problems: formulations, properties and heuristic algorithms. Transportation Science, 26, 185-200.

69. Maurizio Bruglieri, Ferdinando Pezzella, Ornella Pisacane, \& Stefano Suraci (2015). A Variable Neighborhood Search Branching for the Electric Vehicle Routing Problem with Time Windows, Electronic Notes in Discrete Mathematics, 47, 221-228.

70. McMorrin F., R. Anderson, I. Featherstone, C. Watson. (2012). Pluuged-in fleets: A guide to deploying electric vehicle in fleets. The Climate Group, Cenex, and Energy Saving Trust. URL http://www.theclimategroup.org/_assets/files/EV_report_final_hi-res.pdf. (Accessed September 2015).

71. Mendoza, J. E., Castanier, B., Guéret, C., Medaglia, A. L., \& Velasco, N. (2010). A memetic algorithm for the multi-compartment vehicle routing problem with stochastic demands. Computers \& Operations Research, 37, 1886-1898.

72. Nagy, G., \& Salhi, S. (2005). Heuristic algorithm for single and multiple depot vehicle routing problems with pickups and deliveries. European Journal of Operational Research, 162, 126-141.

73. Ons Sassi, Wahiba Ramdane Cherif, Ammar Oulamara. Vehicle Routing Problem with Mixed fleet of conventional and heterogenous electric vehicles and time dependent charging costs. 2014. URL https://hal.archives-ouvertes.fr/hal-

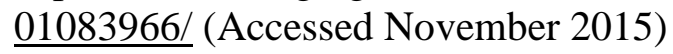


74. Palmer, A. (2007). The development of an integrated routing and carbon dioxide emissions model for goods vehicles. PhD thesis. Bedford, United Kingdom: Cranfield University.

75. Polacek, M., Hartl, R. F., Doerner, K., \& Reimann, M. (2004). A variable neighborhood search for the multi depot vehicle routing problem with time windows. Journal of Heuristics, 10, 613-627.

76. Potvin, J. Y., J. M. Rousseau. (1993). A parallel route building algorithm for the vehicle routing scheduling problem with time windows. European Journal of Operations Research, 66, 331-340.

77. Qian, J. (2012). Fuel emission optimization in vehicle routing problems with timevarying speeds. PhD thesis. Lancaster, United Kingdom: Lancaster University.

78. Salhi, S., \& Sari, M. (1997). A multi-level composite heuristic for the multi-depot vehicle fleet mix problem. European Journal of Operational Research, 103, 95112.

79. Salhi, S., Imran, A., \& Wassan, N. A. (2013). The multi-depot vehicle routing problem with heterogeneous vehicle fleet: Formulation and a variable neighborhood search implementation. Computers \& Operations Research Part B, 52, 315-325.

80. Sbihi, A., \& Eglese, R.W. (2007). The relationship between vehicle routing \& scheduling and green logistics-A literature survey. Technical report, Lancaster University Management School, United Kingdom. http://halshs.archivesouvertes.fr/hal-00644133/ (Accessed November 2015).

81. Schneider, M., Stenger, A., Goeke, D. (2014). The Electric Vehicle Routing Problem with Time Windows and Recharging Stations. Transportation Science.

82. Schrimpf, G., J. Schneider, H. Stamm-Wilmbrandt, G. Dueck. (2000). Record breaking optimization results using the ruin and recreate principle. Journal of Computational Physics, 159, 139-171.

83. Sevgi Erdoğan,\& Elise Miller-Hooks (2012). A Green Vehicle Routing Problem, Transportation Research Part E: Logistics and Transportation Review, 48(1), 100114.

84. Soler, D., Albiach, J., \& Martínez, E. (2009). A way to optimally solve a timedependent vehicle routing problem with time windows. Operations Research Letters, 37(1), 37-42.

85. Solomon, M. M. (1987). Algorithms for the vehicle routing and scheduling problems with time window constraints. Operations Research, 35, 254-265.

86. Taefi T., J. Kreutzfeldt, T. Held, R. Konings, R. Kotter, S. Lilley, H. Baster, N. Green, M. Stie Laugesen, S. Jacobsson, M. Borgqvist, C. Nyquist. (2014). Comparative analysis of European examples of freight electric vehicles scheme. A systematic case study approach with examples from Denmark, Germany, the Netherlands, Sweden, and the UK. 4th International Conference on Dynamic in Logistics (LDIC 2014). Bremen, Germany.

87. Tavares, G., Zsigraiova, Z., Semiao, V., \& Carvalho, M. G. (2009). Optimisation of MSW collection routes for minimum fuel consumption using 3D GIS modelling. Waste Management, 29(3), 1176-1185. 
88. Tavares, G., Zsigraiova, Z., Semiao, V., \& da Graça Carvalho, M. (2008). A case study of fuel savings through optimisation of MSW transportation routes. Management of Environmental Quality, 19(4), 444-454.

89. Tillman, F. A. (1969). The multiple terminal delivery problem with probabilistic demands. Transportation Science, 3, 192-204.

90. United Nations Environment Programme. UNEP environmental assessment Expo $2010 \quad$ Shanghai, China (2010). URL http://www.unep.org/pdf/SHANGHAI_REPORT_FullReport.pdf. (Accessed October 2015)

91. US DOT, Department of Transportation, 2010. The Transportation's Role in Reducing US Greenhouse Gas Emissions. URL

92. vanKeulen,T., deJager, B., Serrarens, A., \& Steinbuch, M. (2010). Optimal energy management in hybrid electric trucks using route information. Oil and Gas Science and Technology, 65(1), 103-113.

93. Watson-Gandy, C., \& Dohm, P. (1973). Depot location with van salesmen - a practical approach. Omega, 1, 321-329.

94. Wilson, H., \& Weissberg, H. (1967). Advanced dial-a-ride algorithms research project: final report. Technical Report. R76-20, Department of Civil Engineering, MIT, Cambridge, MA.

95. X iao, Y., Zhao, Q., Kaku, I., \& Xu, Y. (2012). Development of a fuel consumption optimization model for the capacitated vehicle routing problem. Computers \& Operations Research, 39(7), 1419-1431.

96. Yang, J., Jaillet, P., \& Mahmassani, H. (2004). Real-time multivehicle truckload pickup and delivery problems. Transportation Science, 38, 135-148. 